\title{
APPENDIX
}

TO THE

\section{Consolidated Annual Implementation Plan}

Second Year - 1991/92

Cooperative Agreement No. 306-0204-A-00-0767-00

\author{
between
}

Office of the A.I.D. Representative to Afghanistan

and Volunteers in Technical Assistance

for the Implementation of the

Agriculture Sector Support Project

Agriculture and Rural Rehabilitation

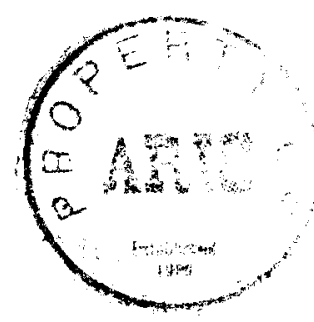

May 30, 1991 


\section{APPENDIX}

A. PROGRESS AND PROPOSED SCHEDULE

A $1-17$

B. ANNUAL IRRIGATION PLAN 1991/92 - FORM IR-1

C. ANNUAL IRRIGATION PLAN 1991/92 - FORM IR-2

C $1-16$

D. ANNUAL ROADS AND BRIDGES PLAN 1991/92 - FORM RB-1

D

E. ANNUAL ROADS AND BRIDGES PLAN 1991/92 - FORM RB-2

E 1-16

F. VITA/LLBI CONTRACT - SCOPE OF WORK

G. TRAINING PLAN 1990/91

H. VITA / CID CONTRACT - SCOPE OF WORK

H 1-10

I. SUBPROJECT MONITORING FORM

J. MONITORING FORM REVISED

K. LEVEL-1 MONITORING FORM

L. LEVEL-3 MONITORING FORM

M. RURAL WORKS INSPECTION FORM

M 1-6

N. MIT CONTRACT SCOPE OF WORK

N 1-9

O. LIST OF AGENCIES ASSISTING AFGHANISTAN

O $1-2$

P. SCOPE OF WORK, CONSULTANT ALDELMO RUIZ

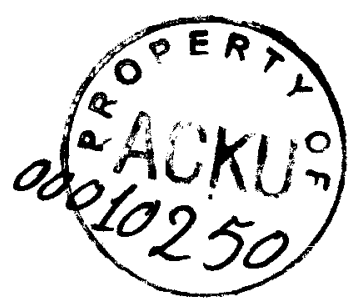


I. Province - (01) BABUL

ARS Started: March, 1989. Started work Sept, 1988.

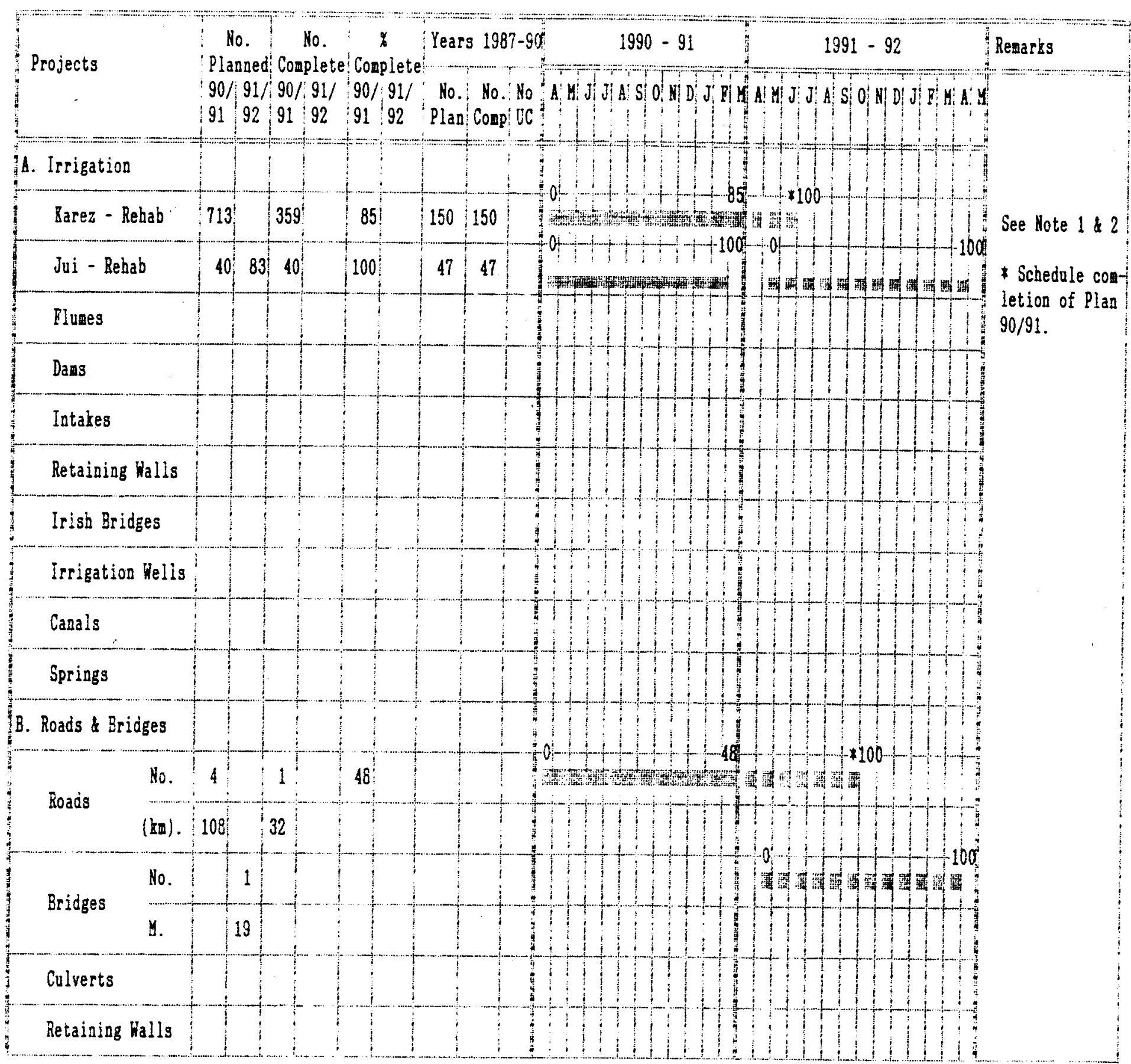

Scheduled - in NOTSS:

Actual:

Scheduled:

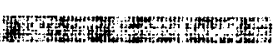

LEGEHD:

BTC= Bstinate To Couplete

$U C=$ Under Construction

1991-92 - Curreat Plan
1. Scheciule includes car:y over project' for Plan 30/91.

2. An additional 366 projects vere add- $1987 / 90: 197 \quad 197$ ed at a later date to the originai Plan for a total of 713 projects.

3. Bstimate includes BTC.
Ponds (in Rupees)

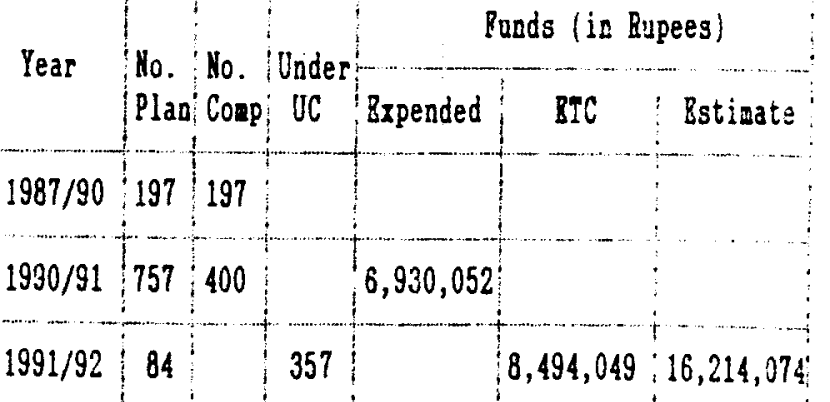


PROGRESS \& PROPOSED SCHEDULE

CONSTRUCTION \& BRBCTIOH

AGRICULTURB SECTOR SUPPORT

I. Province - ( 02) XAPISA

Agriculture \& Rural Rehabilitation Project Subnitted by: Eng. Shahzada

ARS Dir. USAID/VITA

ARS Started: June, 1990

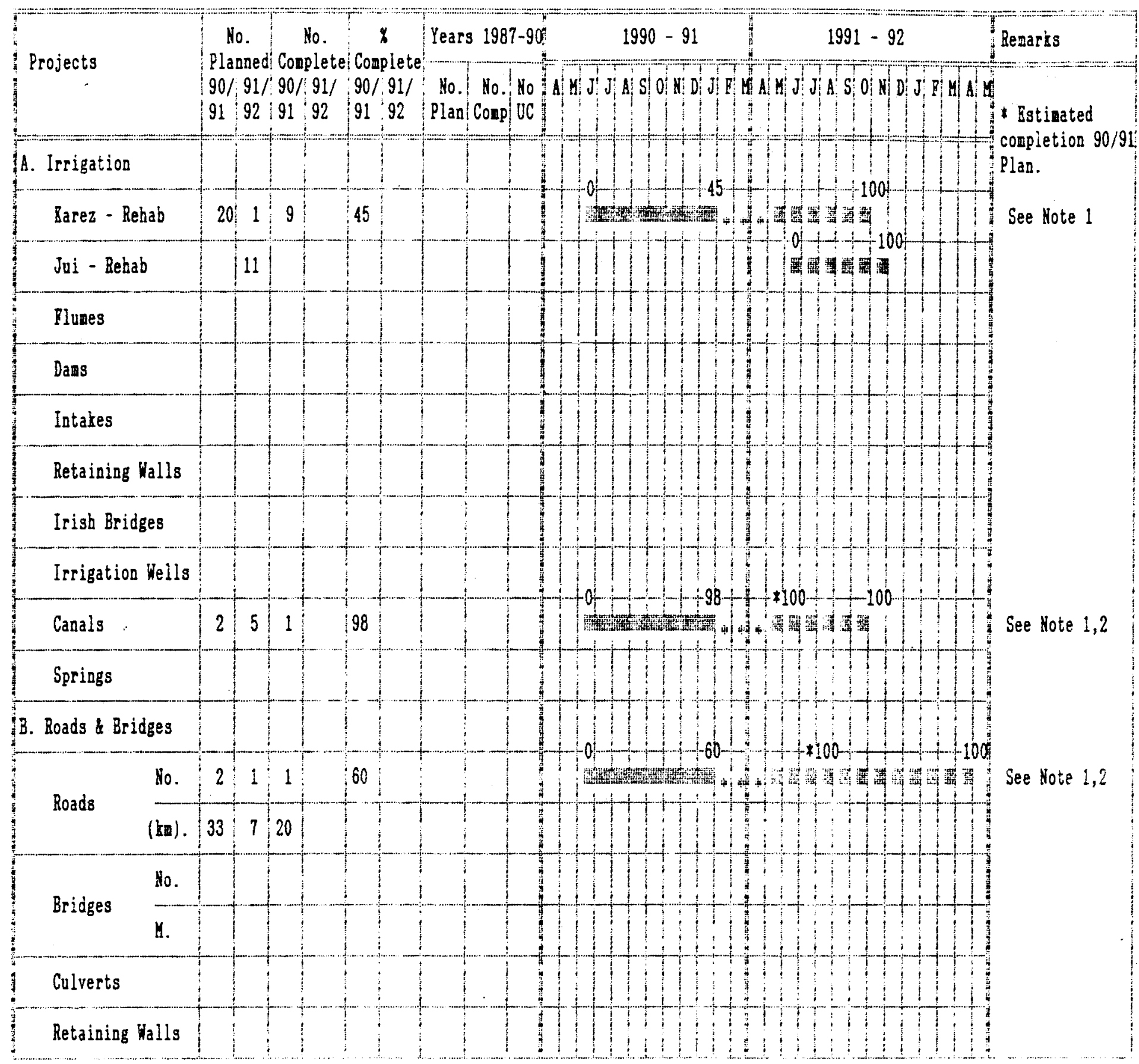

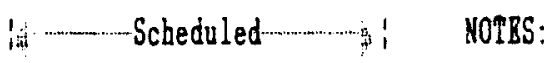

Progress:

Actual:

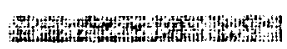

Scheduled:

LBGEHD:

BTC= Bstizate to complete

UC = Under Construction

1991-92 - Current Plan
1. Whory stop due to lack of Funds.

2. Schedule includes carry-over projects $1990 / 91$.

3. Delayed construction Bridge Plan 90/91 because cable is not available in Afghanistan $91 / 92$ Bridges are Plan to be concrete.

4. Bstinate includes BTC.
Punds (in Rupees)

Year

No. No. Under

Plan Comp UC Bxpended BTC Bstinate

$1987 / 90$

\begin{tabular}{|l|l|l|l|l}
$1990 / 91$ & 24 & 11 & $1,379,637$
\end{tabular}

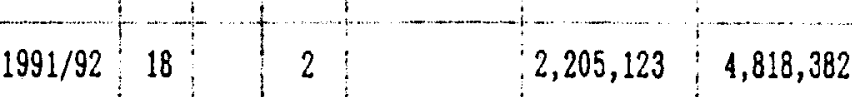


1. Province - 103$)$ ParwaN Agriculture \& Rural Rehabilitation Project USAID/VITA

ARS Started: July, 1989

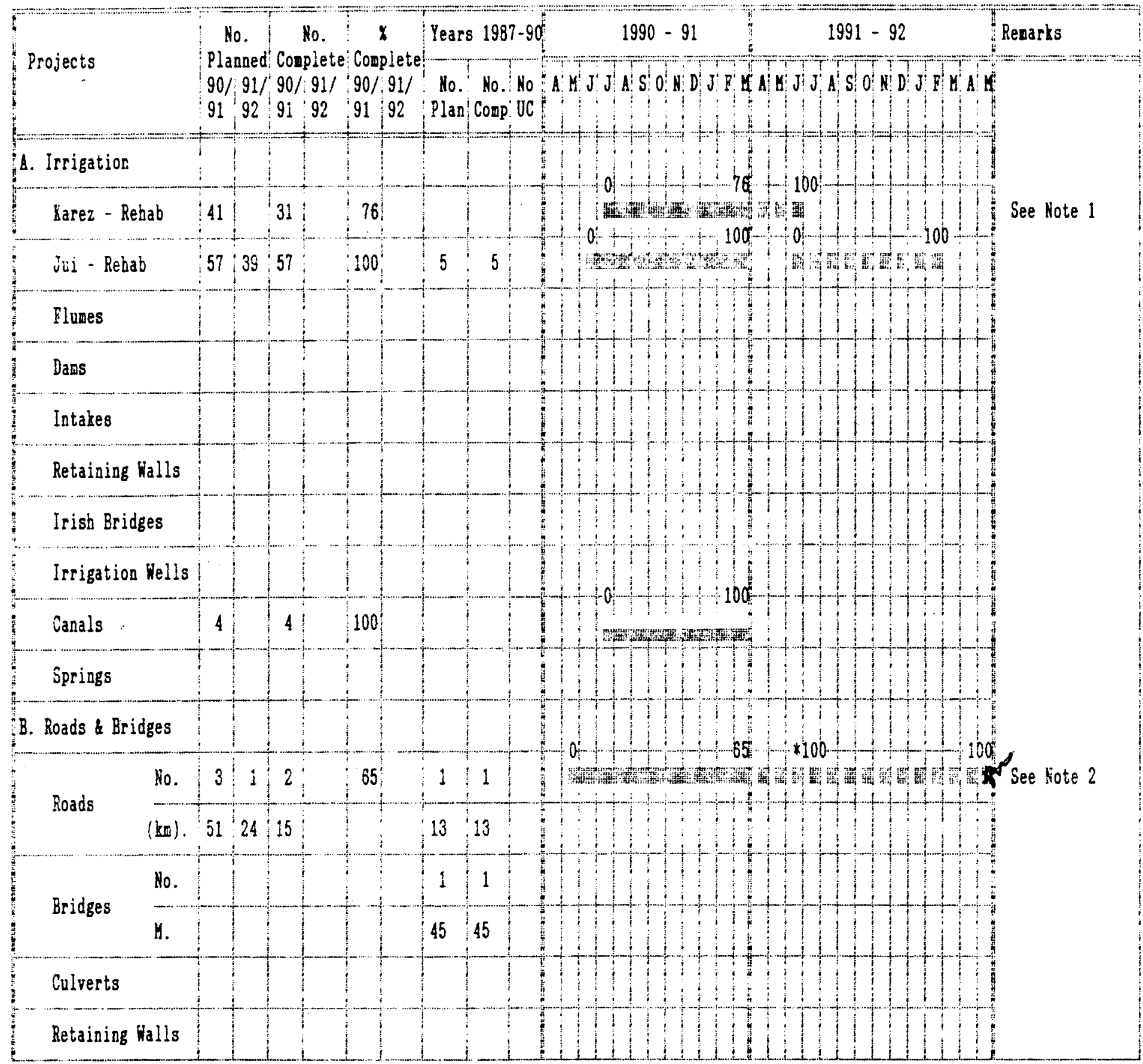

Scheduled-... I NOREs:

Progress:

Actual:

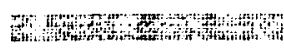

Scheduled:

LEGBND:

BTC= Bstinate Io Complete

UC = Under Construction

1991-92 - Current Plan
1. $x$ Completion reflects progress on or-going projects.

2. Indicates estimated date coniet-1987/90:7:7: ion 90/91 Pian.

4. Bstinate includes BTC.
Iunds (in Rupees)

Year Subwitted by: Eng. Hoho Daud

Ho. No. Under:

Plan:Comp UC Expended BTC

Estinate

$\begin{array}{l:l:l:l}1990 / 9: & 105 & 94 & 3,801,771\end{array}$

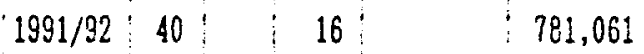

$2,732,520$ 
PROGRRSS \& PROPOSBD SCHBDULB

CONSTRUCTION \& BRRCTION

AGRICULTORR SBCTOR SUPPORT

I. Province - (04) HARDAS

Agriculture \& Rural Rehabilitation Project

USAID/NITA

ARS Started: July, 1987.

No. Ho. $\%$ Years 1987-90 $1990-91$

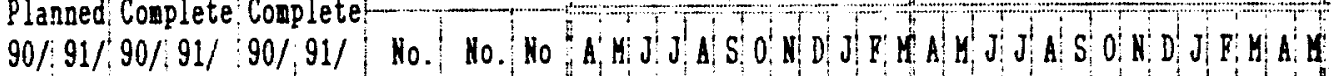

$91,92: 91,92 \quad 91,92 \quad$ Plan Conp OC

A. Irrigation

Rarez - Kebab

$131: 287 \quad 99$

496 340 156

See Note 1

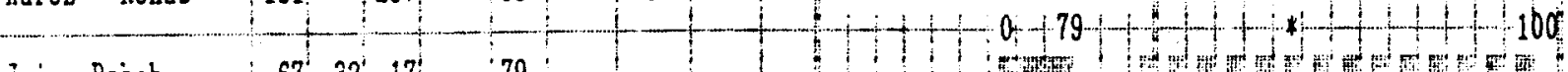

Jui-Reáab $67,32: 17:$

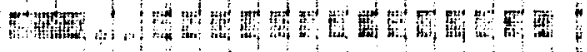

See Note $2,3,4$

Fiumes

Dams

Intakes

$87: \quad 3: 26$

100

$10-100-1+10+1+++100$

Retaining Walls

Irish Bridges

Irrigation Weils

Canals

Springs

30

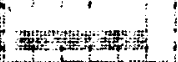

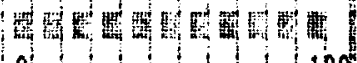

Remaris

B. Roads \& Bridges

No.

Roads

(kn).

No. $4: 154:$

100

Bridges

म. $80: 380,80$

Culverts

Retaining Walls

in - Scheduled -..... NOTBS:

Progress:

Actual:

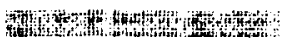

Scineduled

LBGEND:

BTC= Bstimate To Complete

$U C=$ Under Construction

1991-92 - Current Plan
1. No. Conplete includes projects carried over fron years $1987-90$ Fian.

2. Worls stop on at the end of Decenber 90 to the end of Jan, 91 due to the weather.

3. : conpleted reflects only projects approved.

4. Estirate includes EIC.
Year Ho. Ho. Under $\quad$ Punds (in Rupees)

'Plan Comp OC Expended; BTC Bstivate

$\begin{array}{llllll}1987 / 90 & 496 & 340 & 156\end{array}$

\begin{tabular}{l|l|l|l|l|}
\hline $1990 / 91$ & 444 & 375 & $2,125,616$ \\
\hline
\end{tabular}

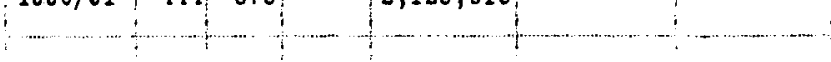

\begin{tabular}{|l|l|l|l|l|l|l}
\hline $1991 / 92$ & 80 & 0 & 11 & $1,000,884$ & $3,400,884$
\end{tabular} 
I. Province - ( 05) LOGAR

ARS Started: Dec, 1987
Agriculture \& Rural Rehabilitation Project USAID/VITA

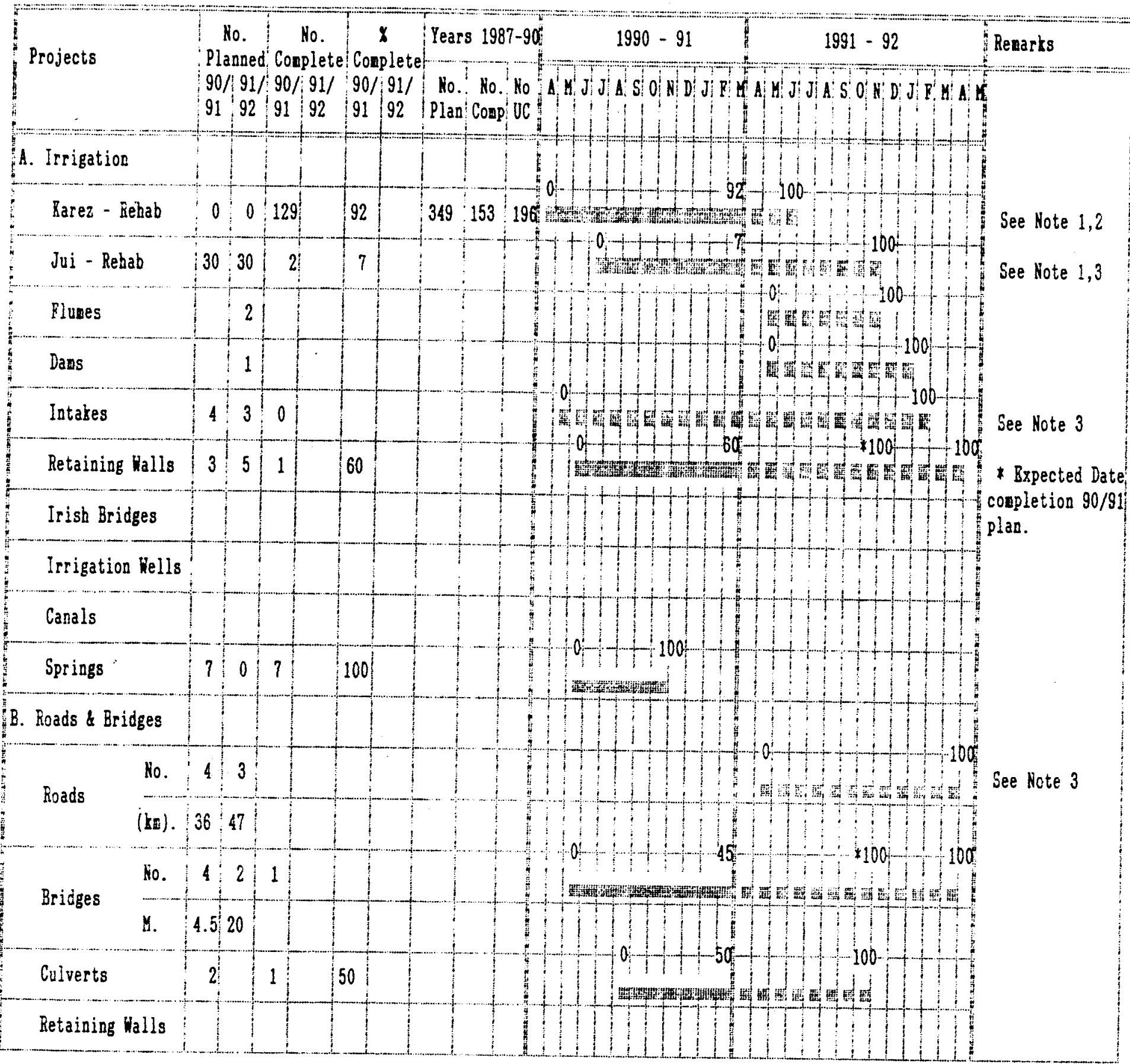

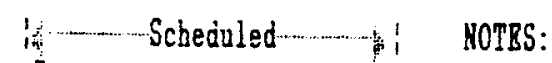

Progress:

Actual:

75y

Scheduied:

LEGEHD:

BTC= Istimate Io Conplete

$\forall C=$ Onder Construction

$1990-91-$ Previous Plan $=109$

$1991-92-$ Current Plan $=29$

1. : Complete includes progress on on going projects.

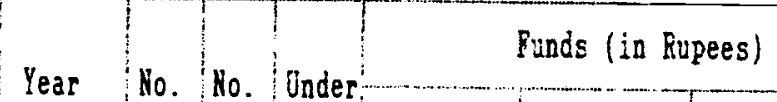

Plan Comp UC Expended BTC Estimate

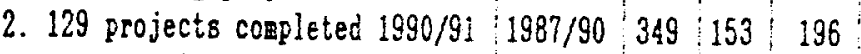
were carried over frob previous years $87 / 90$.

3. ?rojects progress delayed due to late approval.

4. Istinate inciudes RPC.

\begin{tabular}{ll:l|l}
\hline $1990 / 91$ & 54 & 141 & $2,025,408$
\end{tabular}

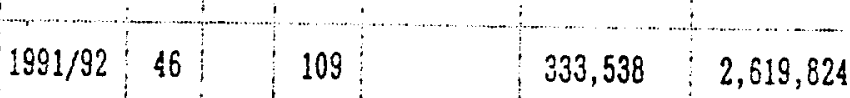


ARS Started: May, 1988 USAID/VITA

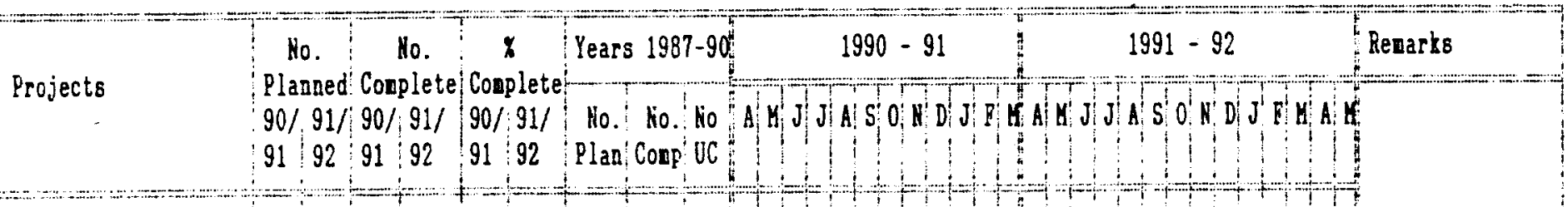

A. Irrigation

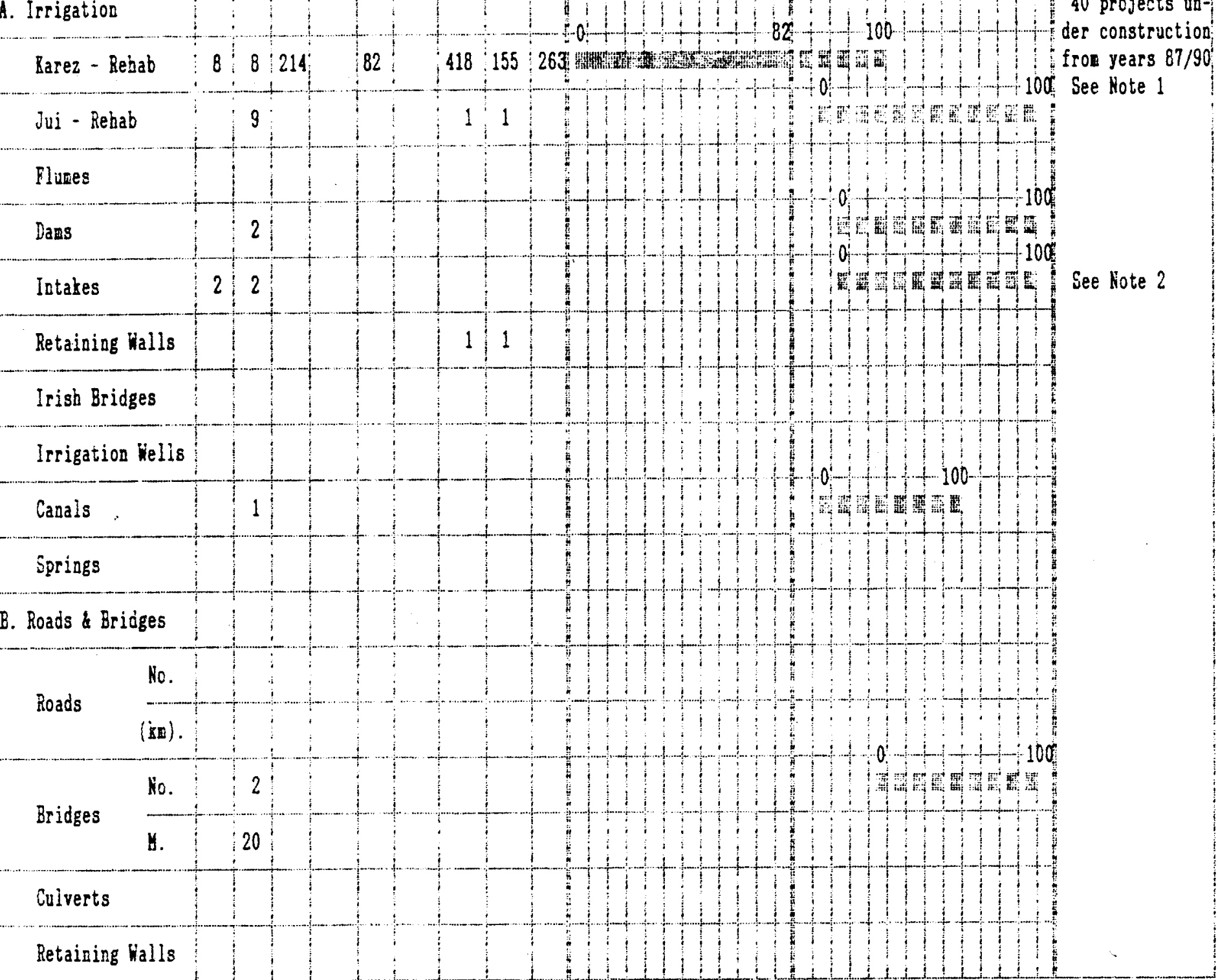

Scheduled - HOIBS:

Progress:

Actual:

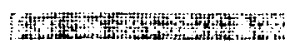

Scheciuled:

I

Lagnd:

BTC = Istimated To Complete

$U C=$ Under Construction

1991-92 - Current Plan
1. Xomplete reflects coupletion of projects carried-over from 1987-90 Plan.

2. Intake were approved on $1 / 23 / 91$

3. Estimate includes BIC.

\section{Funds (in Rupees)}

Year

Ho. No. Under
Plan Coup UC Bxpended

$1987 / 90 \quad 420 \quad 157 \div 263$

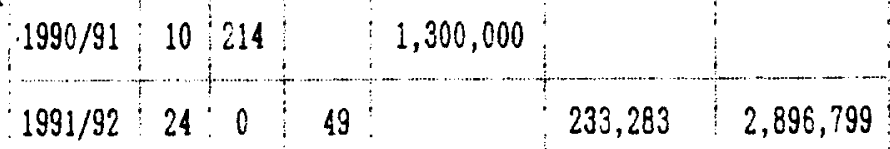


ARS Started: July, 1989, Started work Aug, 1987.

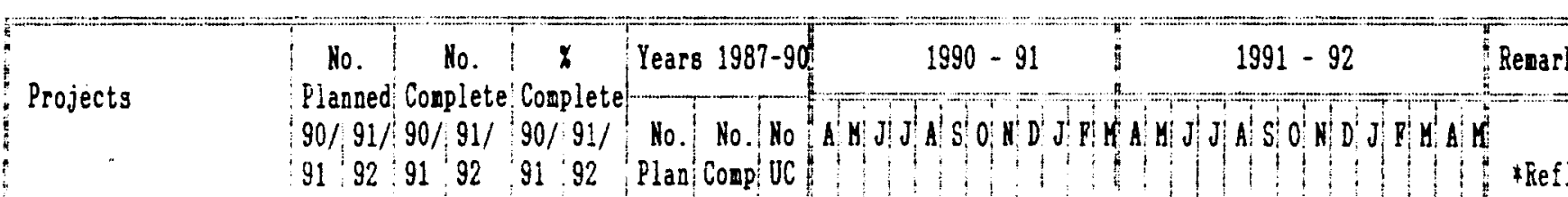

A. Irrigation

$\frac{\text { Barez - Rehab }}{\text { jui - Rehab }}$

$137,17 \quad 50$

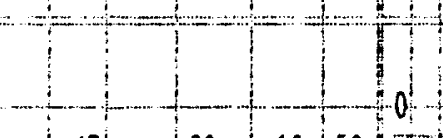

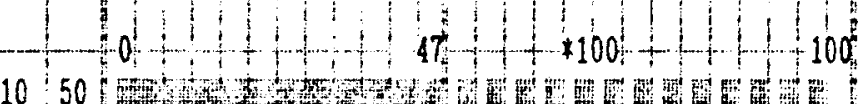

\section{Ilunes}

Dans

Intares

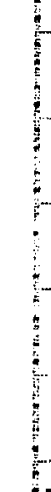

Retaining Malls

Irish Bridges

Irrigation Wells

Canals

Springs

B. Roads \& Bridges

Roads

No.

(kD)

$24: 41 ; 21$ $92:$

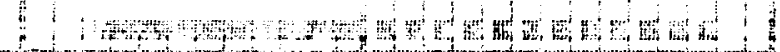

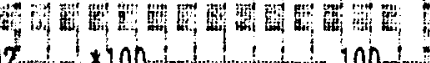

Keflects conpletion Planned 90/91.

See Hote 1,2

See Note 1,2 Subnitted by: Ghuiar Sarhi Baaz ARS Dir. 
I. Province - (10) SOHAR

Agriculture \& Rural Rebabilitation Project USAID/VITA

ARS Started: Hov, 1987

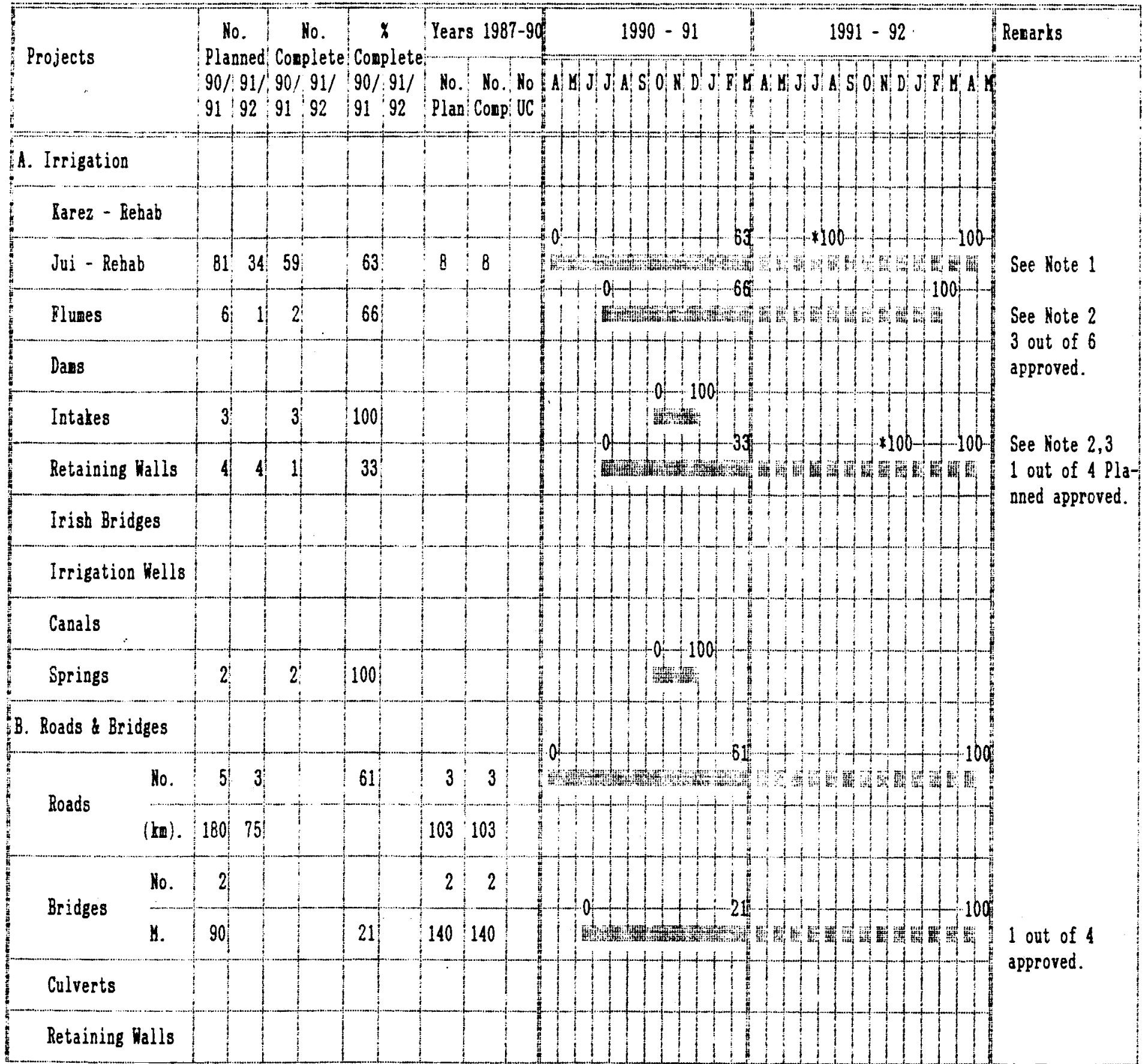

in Scheduled-and NOTBS:

Progress:

Actual:

Fintions

Scheduled:

LEGRND:
1. * Complete reflects progress on on-going projects.

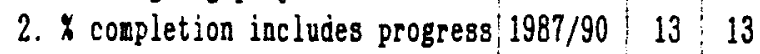
or approved projects only.

3. * Jate of completion 90/91 Plan projects.

4. Bstimate includes ETC.

\section{Funds (in Rupees)}

Year

Ho. No. Under

PlaniCon UC Expended

BTC

Estinate

\begin{tabular}{l|l:l|l|l}
$1990 / 91$ & 103 & 67 & & $8.373,990$ \\
\hline
\end{tabular}

\begin{tabular}{|l|l|l|l|l|l|}
\hline & & & & \\
\hline $1991 / 92$ & 42 & 10 & & $4,840,161$ & $7,501,018$ \\
\hline
\end{tabular}

BTC= Bstimate To Complete $U C=$ Under Construction 1991-92 - Current Plan 
I. Province - ( 12) TAYHAR

Agriculture \& Rural Rehabilitation Project

USAID/NITA

ARS Started: Sept, 1987

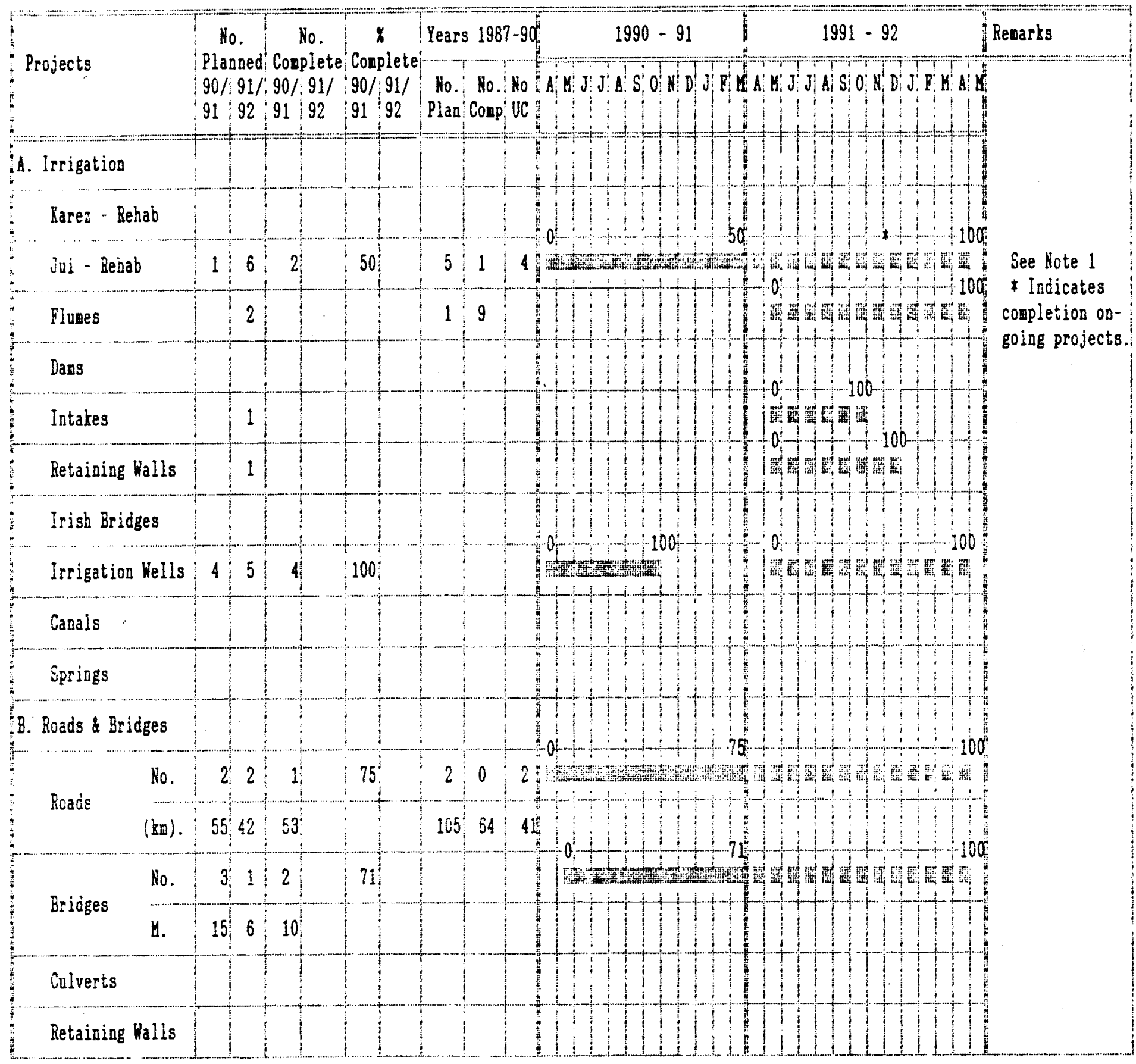

1......... Scheduled - NOT̈BS:

\section{Progrëss:}

Actual:

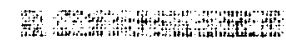

Scheduled:

LEGEND:
1. Ho. complete year $90 / 91$ shows 1 project conplete fron prior years 87/90. \% conplete indica- $1987 / 90: 8$ 10:6 tes progress years $89 / 9090 / 91$ Plans.

2. Bstinate includes ETC. 
PROGRRSS \& PROPOSED SCHBDOLR

CONSTROCTION \& BRECTION

AGRICULTURE SECTOR SUPPORT

I. Province - (13) BAGHLAR

ARS Started: ₹eb, 1989

Agriculture \& Rural Rehabilitation Project Suboitted by: Bng. Mohd. Ashraf ARS Dir. USAID/VITA

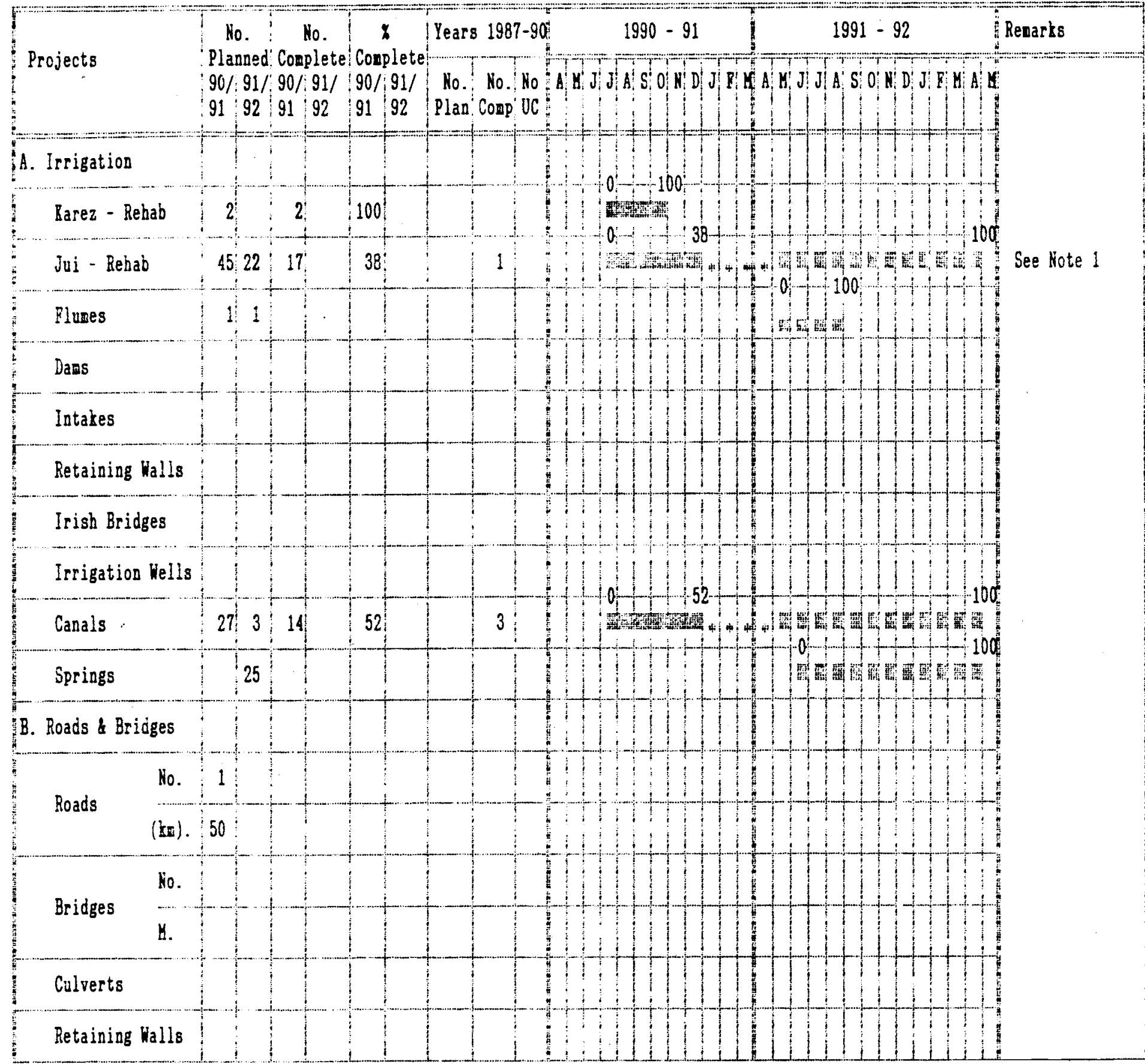

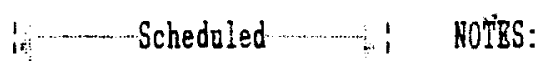

Progress: .

Actual:

At
Scheouled:

LEGEND:

BTC= Bstimate To Complete

$U C=$ Onder Construction

1991-92 - Current Plan
1. Work stop on Dec $20 / 90$ and Director departed for Peshawar:and renained until end of $1987 / 90$ April, 1991.

2. Plan for $91 / 92$ taikes into con:- $1990 / 9176: 33$ sideration tire delays on projects approval.

3. Estimate includes BIC.

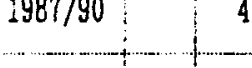

Punds (in Rupees)

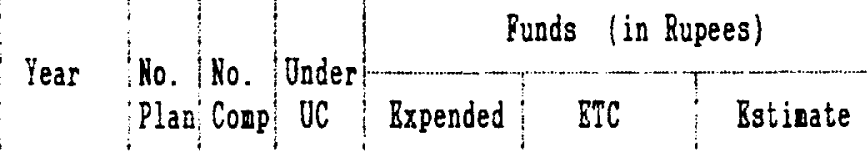

$: 1991 / 92: 51 \quad 16 \quad 1,303,846: 3,626,131$


PROGRESS \& PROPOSED SCHKDULE

COHSTRUCTIOH \& BRECTIOY

AGRICULTURE SRCTOR SOPPORT

1. Province - (14) RUWDUZ

Agriculture \& Rural Rebabilitation Project Subnitted by: Bng. Shah Jan

ARS Dir. USAID/VITA

ARS Started: Sept, 1990

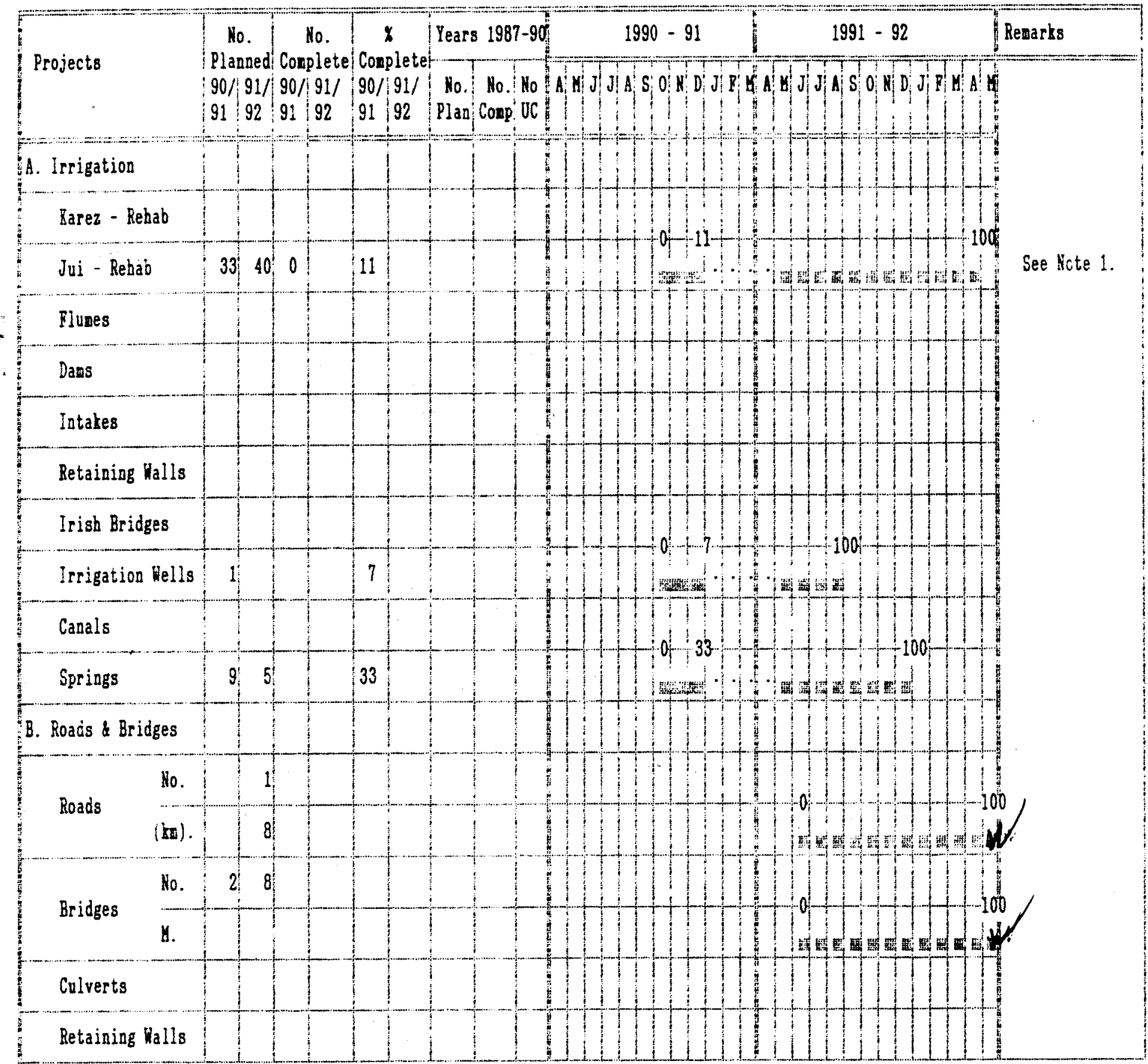

14- Scheduled-ant NOTES:

Progress:

Actual:

Scheduled:

LEGEND:

BTC= Estinate To Complete

UC = Under Construction

1991-92 - Current Plan
1.-....ARS Director arrived Peshawar in December \& remained until end of Plan year. Unaware of progress. 1987/90

2. Bstimate includes BTC.
Punds (in Rupees)

Year

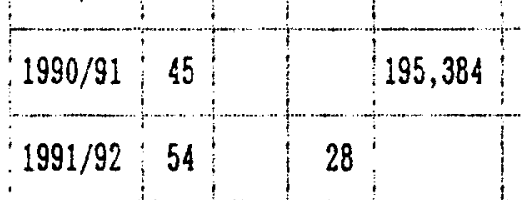

$501,864: 4,156,166$ 
I. Province - (20) HERAT Agriculture \& Rural Rehabilitation Project USAID/VITA

ARS Started: Oct, 1990.

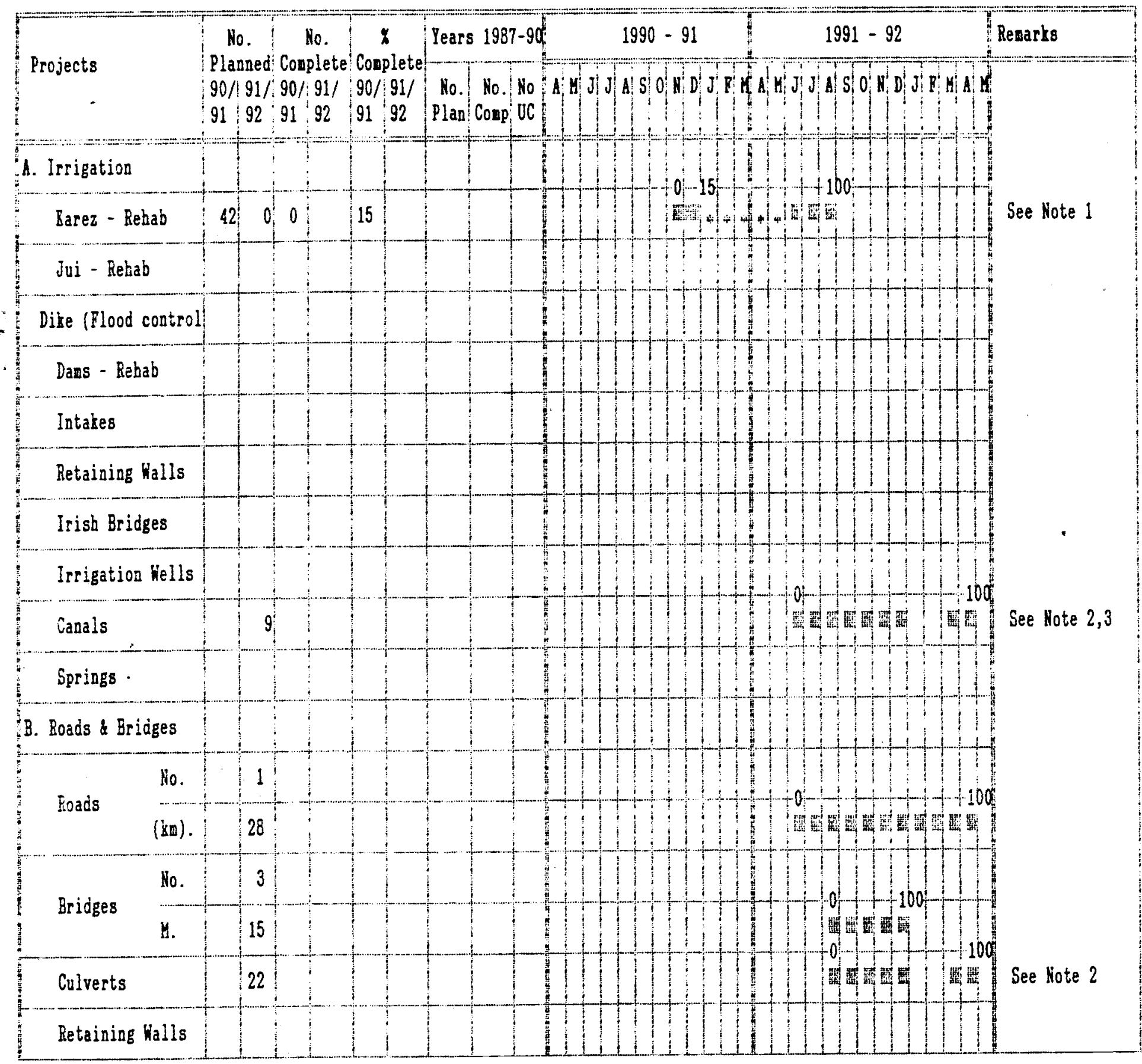

- Scheduled-D- - NOTES:

Progress:

Actual:

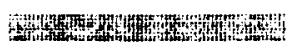

Scheduled:

LRGRND:

BTC= Bstimated To Complete $U C=$ Under Construction 1991-92 - Current Plan
1." Direator in Peshawar since begining of gulf conflict and not aware of field progress.

2. Blant spaces on Schedule indicate vinter nonths when work is discontinued.

3. 9 canals \& 1 road approved in Jan, 1991 while Director in Peshawar.

4. 9 canals \& 1 road approved in Jan, 1991 while Jirector in Peshawar.

5. Istinate includes ETC. Ho. Ho. Under Funds (in Rupees)

Year Plan Conp UC Bxpended BTC Bstinate $1987 / 90$

\begin{tabular}{|l|l|l|l|l|}
\hline $1990 / 91$ & 42 & 0 & 168,008 \\
\hline
\end{tabular}

\begin{tabular}{|l:l:l:l}
\hline $1991 / 92$ & 35 & 0 & 42
\end{tabular} $566,992 \div 7,282,450$ 
I. Province - (21) PARAH Agriculture \& Rural Rehabilitation Project USAID/TITA

ARS Started: Aug, 1990.

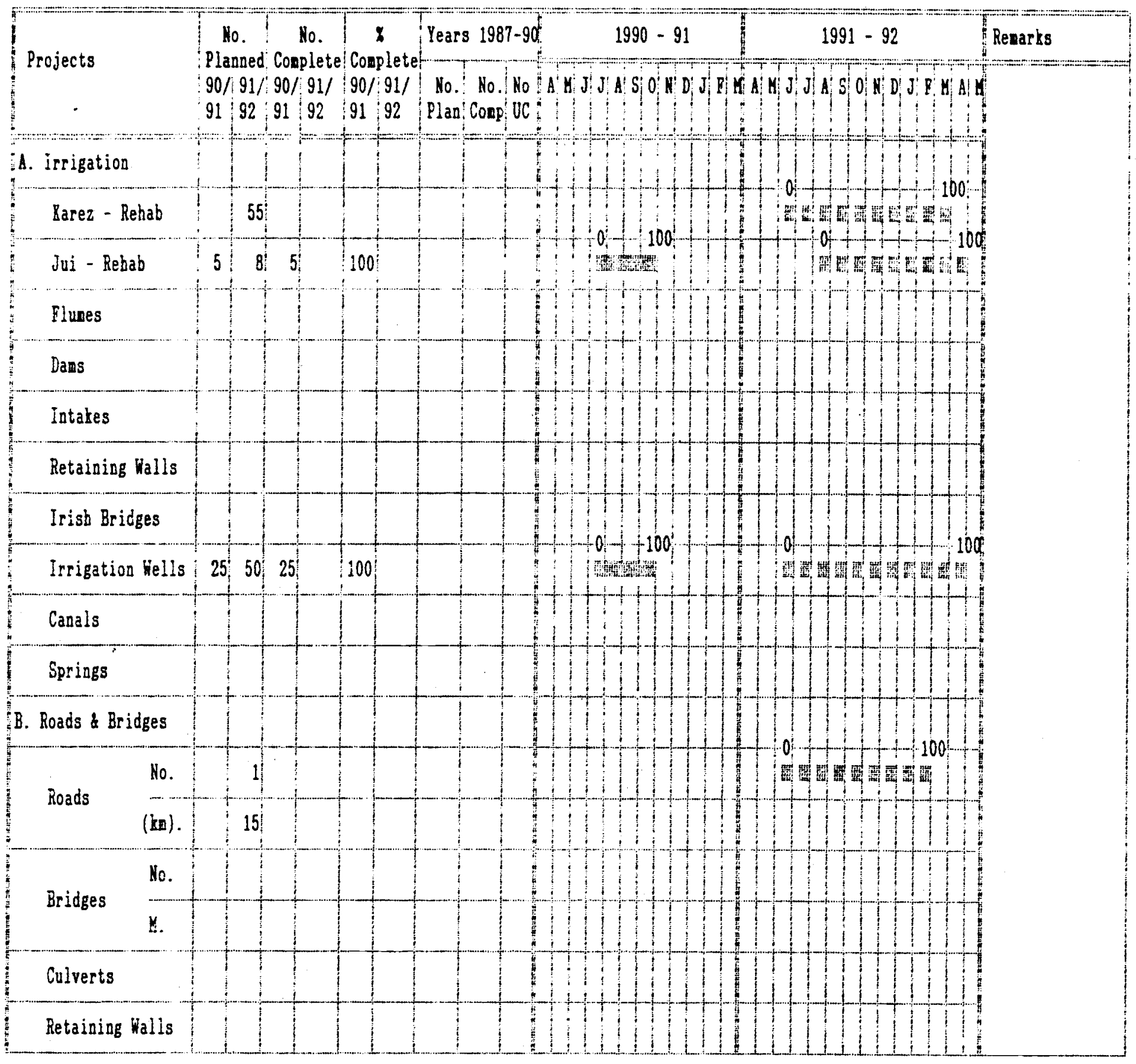

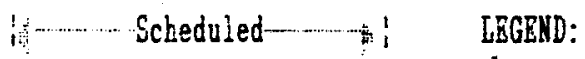

Progress:

Actual:

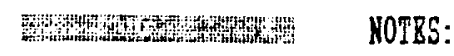

Scheduled:
BIC= Bstinate to Complete $O C=$ Under Construction 1990-91 - Previous Pian 1991-92 - Current Plan

1. Bstimate inciudes BIC

\begin{tabular}{|c|c|c|c|c|c|c|}
\hline \multirow{2}{*}{ Year } & \multirow{2}{*}{$\begin{array}{l}\text { No. } \\
\text { Plan }\end{array}$} & \multirow{2}{*}{$\begin{array}{l}\text { So } \\
\text { Conp }\end{array}$} & \multirow{2}{*}{$\begin{array}{l}\text { Under } \\
\text { OC }\end{array}$} & \multicolumn{3}{|c|}{ Punds (in Rupees) } \\
\hline & & & & Expended & $\mathrm{BTC}$ & Estinate \\
\hline$: 987 / 90$ & & & & & & \\
\hline $\begin{array}{l}1990 / 91 \\
\end{array}$ & 30 & 30 & & 188,530 & & \\
\hline $1991 / 92$ & 114 & & & & & $3,140,011$ \\
\hline
\end{tabular}


PHUGRESS \& PBOPOSED SCHEDULB

CORSTRUCTION \& BRRCTIOH

AGRICULTURR SBCTOR SUPPORT

\section{Province - (24) BAMDAHAR}

ARS Started: Dec, 1987

\section{4}

Agriculture \& Rural Rehabilitation Project Subaitted by: Eng. Sarajudidin ARS Dir. USAID/VITA

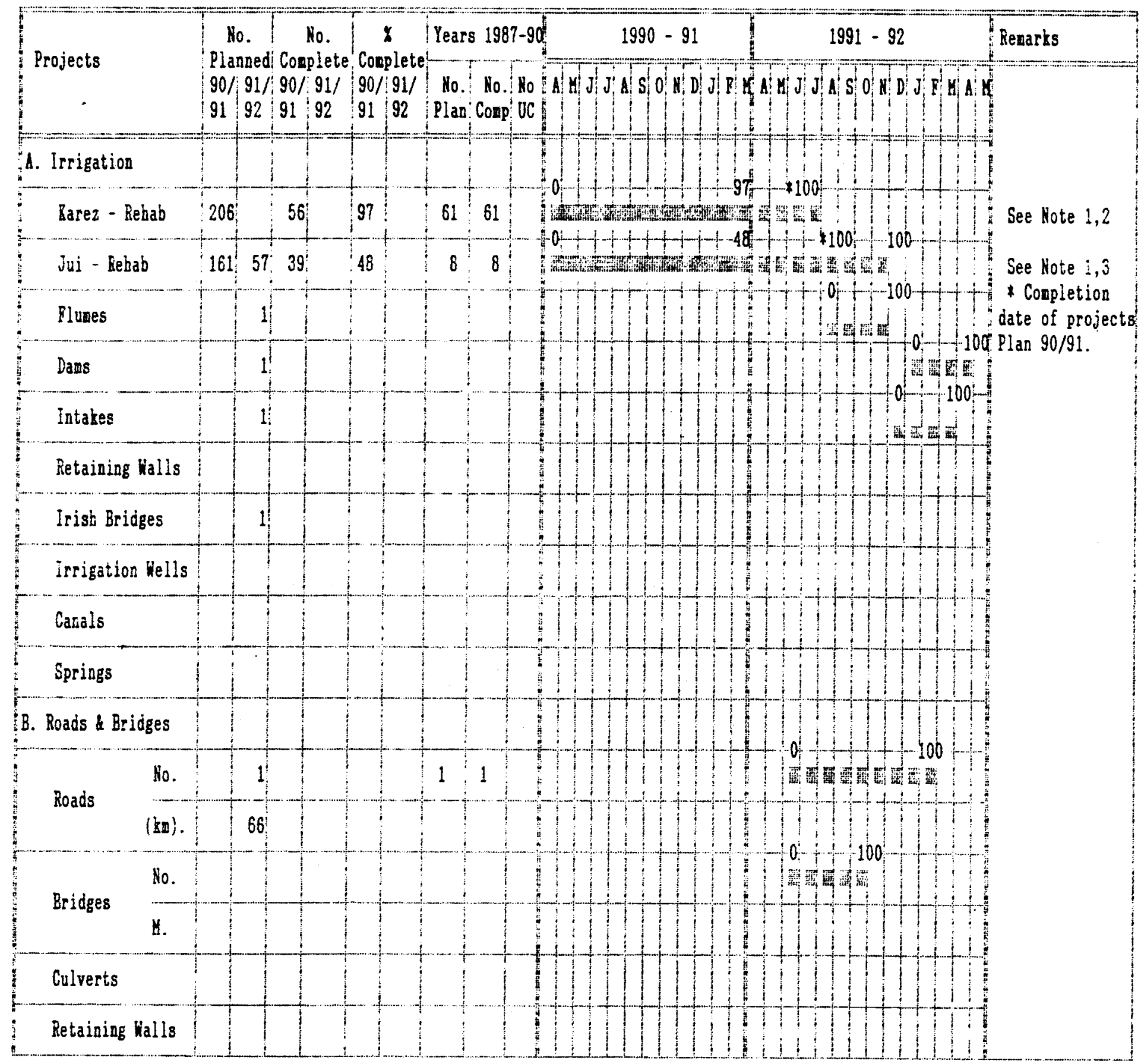

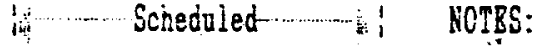

$0 \quad 10 \quad 45: 70 \quad 100$

政:

Progress:

Actual:

mint

Scheduled:

LEGRD:

BTC= Bstizate To Couplete

US = Under Construction

1991-92 - Current Plan
1. $*$ Complete inciudes progress on on-going projects.

2. Out of 206 Iarezes planred for $1987 / 90: 70: 70$ year 1990/91 only 65 were appr-

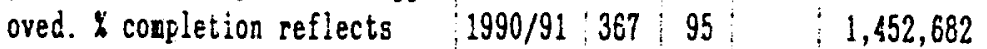
only those projects approved.

3. Out of 161 Jui projects planned: $1991 / 92: 62$ for 1990/91 100 were approved.

oniy 39 projects were complete because work had to be discontinued due to the gulf conflict.

4. Estinate Inciudes BTC.
Year No. No. Onder Punds (in Rupees)
Plan Comp OC Expended ETC Estimate 
I. Province - (28) BAMYAN

AGRICULTURR SBCTOR SUPPOR?

Agriculture \& Rural Rehabilitation Project USAID/VITA

ARS Started: June 1990

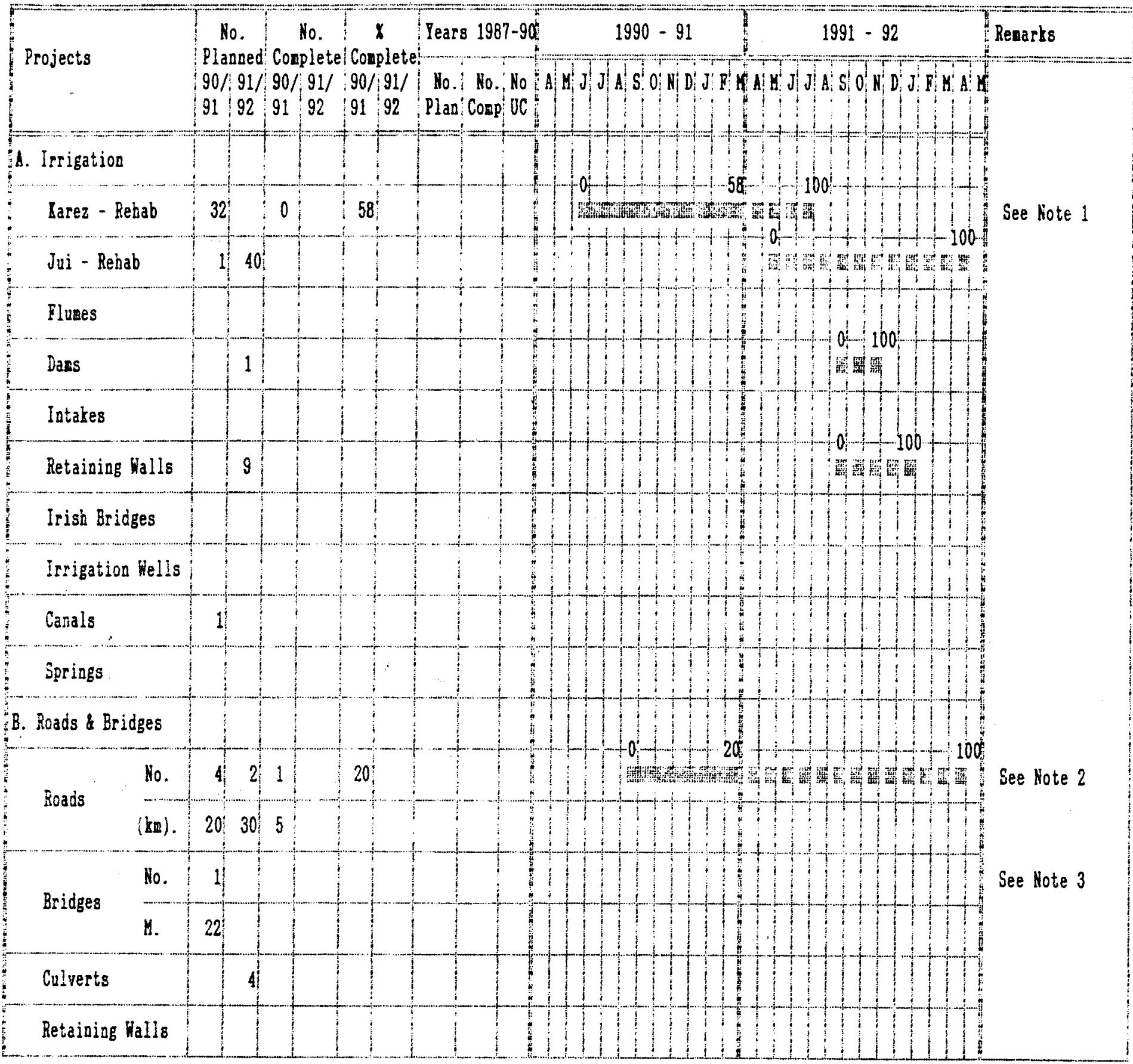

- Scheduled-...- NOTBS:

Progress:

Actual:

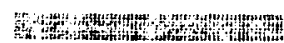

Scheduled:

LRGEND:

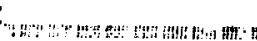

BTC= Estirate Io Complete

UC = Under Construction

:99i-92 - Current Plan
Funds (in Rupees)

1." * Completion reflects progress on on-going projects.

2. Delay due to bed Hinter.

\begin{tabular}{|l|l|l|l|l|l|}
\hline Year & No. No. Onder & \multicolumn{2}{|c|}{ Funds } & (in Rupees) \\
& Plan Conp OC & Expended & BTC & Bstimate \\
\hline $1987 / 90$ & & & & & \\
\hline
\end{tabular}

3. Delay due to lach of construct ion waterials and resent floody $1990 / 91 \quad 39: 1: \quad 260,953$

4. Bstirate includes BIC. 
I. Province - (29) PARTIM

AGRICOLTORR SBCTOR SUPPORT

ARS Started: May, 1987

Agriculture \& Rural Rehabilitation Project Subaitted by: Eng. Janat Gul ARS Dir. USAID/VITA

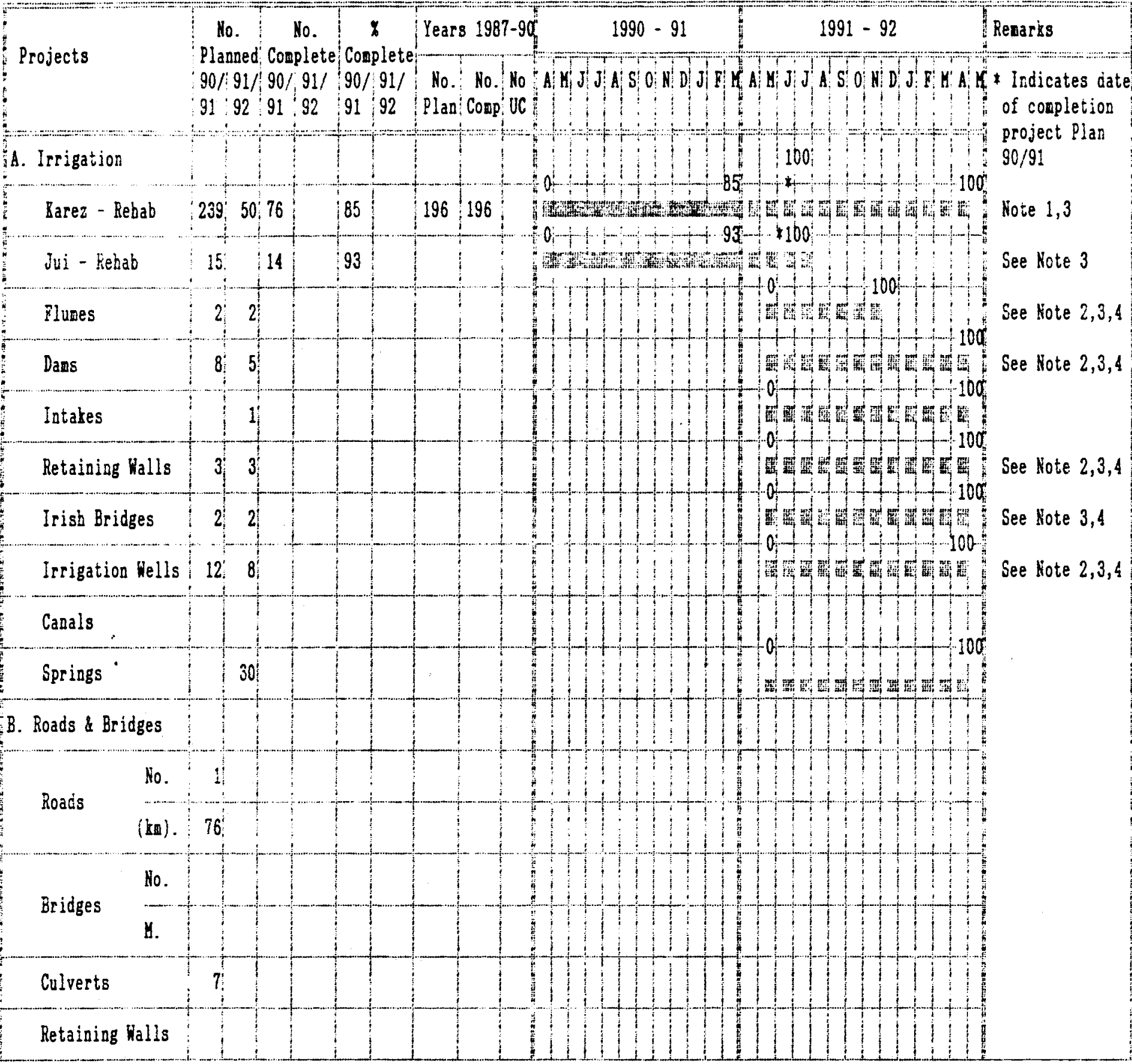

Scheduled-....... NOTES:

\section{Progress:}

Actual:

Scheduled:

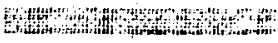

LEGBND:

ETC= Bstimate To Complete

$U C=$ Under Construction

1991-92 - Current Plan
1. $\ddot{*}$ Conplete reflects progress on on-going project.

2. Plans/specfications/cost estirate $1987 / 90$ 196 196

were not ready until $4 / 91$ and aw-

aiting approval to start constru- $1990 / 91289: 90$ ction.

3. Scbedule includes project carried 1991/92 101 over from 90/91.

4. Projects Planned for year $90 / 91$ and not approved are shown in $91 / 92 \mathrm{Plan}$.

5. Bstivate Includes BTC.
Funds (in Rupees)

No. Ho. Under
Plan Cosp UC Expended BTC Estinate

\begin{tabular}{l:l:l} 
& $2,643,744$ & \\
\hdashline 199 & $1,593,000$ & $4,026,208$
\end{tabular}




\section{PROGRRSS \& PROPOSED SCHENOLB \\ CONSTROCTION \& BRECTION \\ AGRICULTURR SECTOR SUPPORT}

Agriclture \& Rural Rehabilitation Project

USAID/VITA

ARR Project/NITA

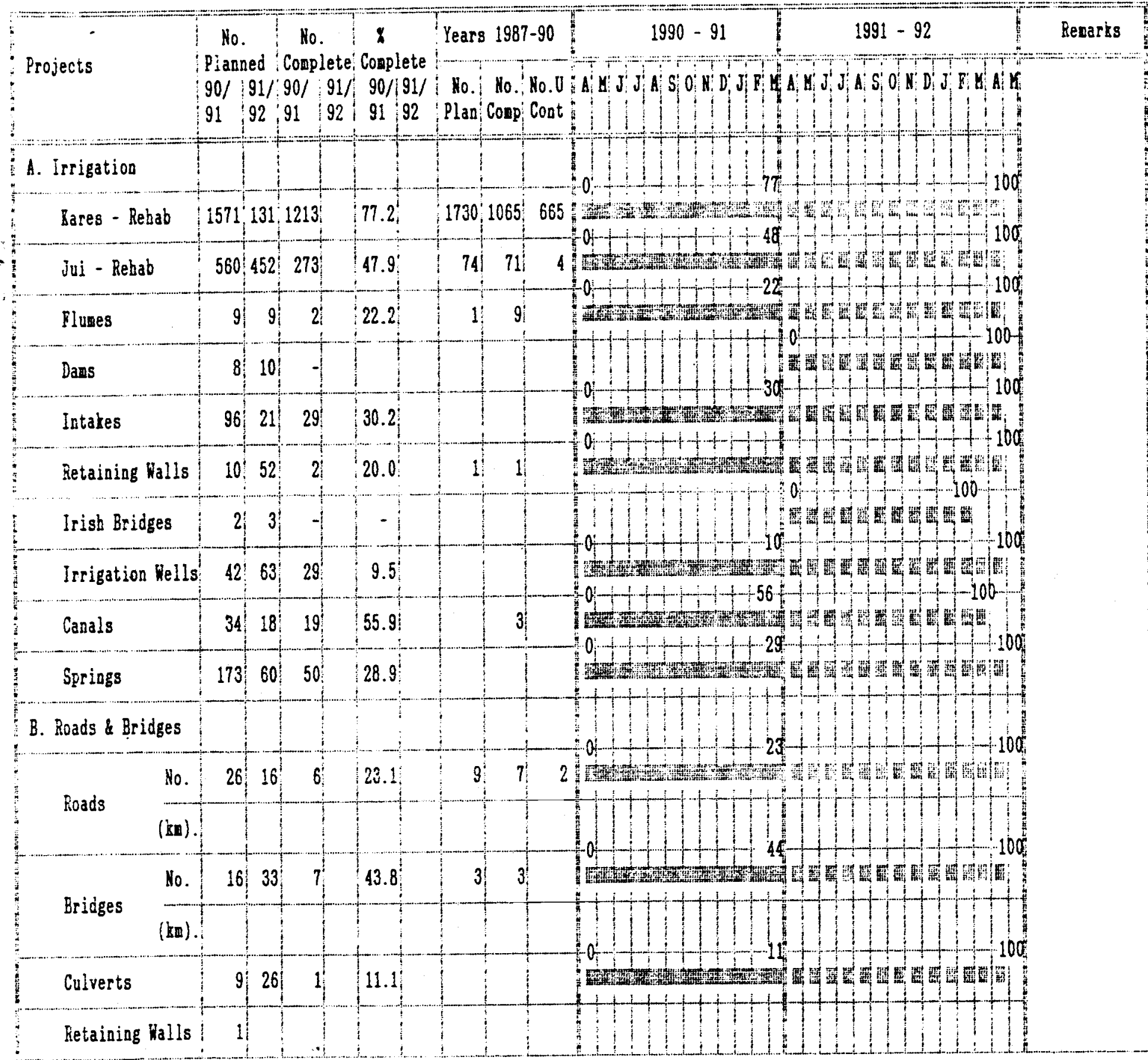

0 - Schedule 100 begend:

ETC = Bstimate To Complete

$U C=$ Under Construction

1991-92 - Current Plan
Punds (in Rupees)

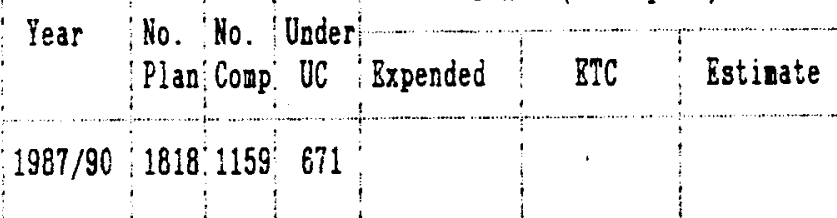

Progress:

$1930 / 91 \quad 2556,1631$

Actual:

$40,476,787$

Scheduled:

$1991 / 92: 894: 387$

$29,498,873: 78,728,081$ 


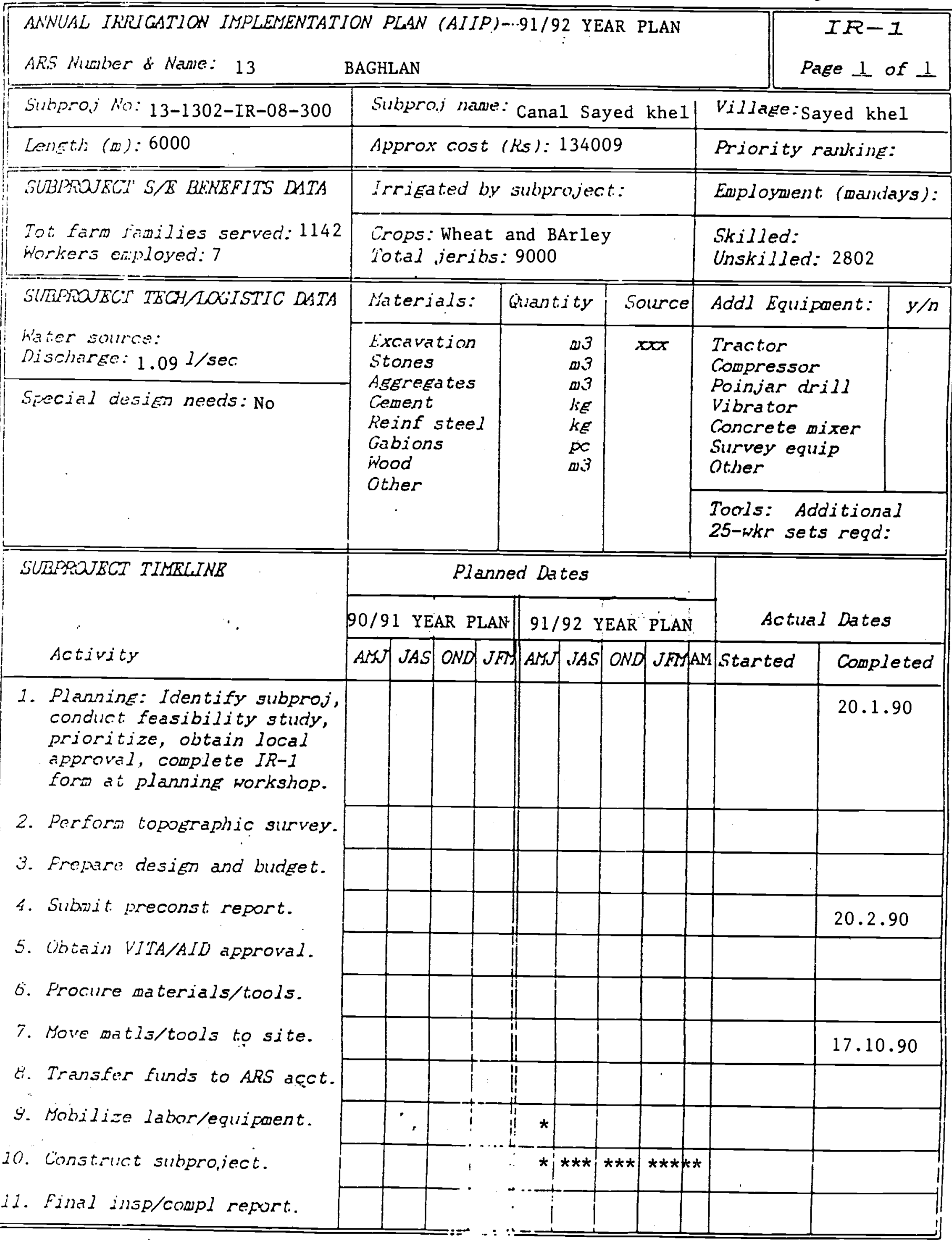




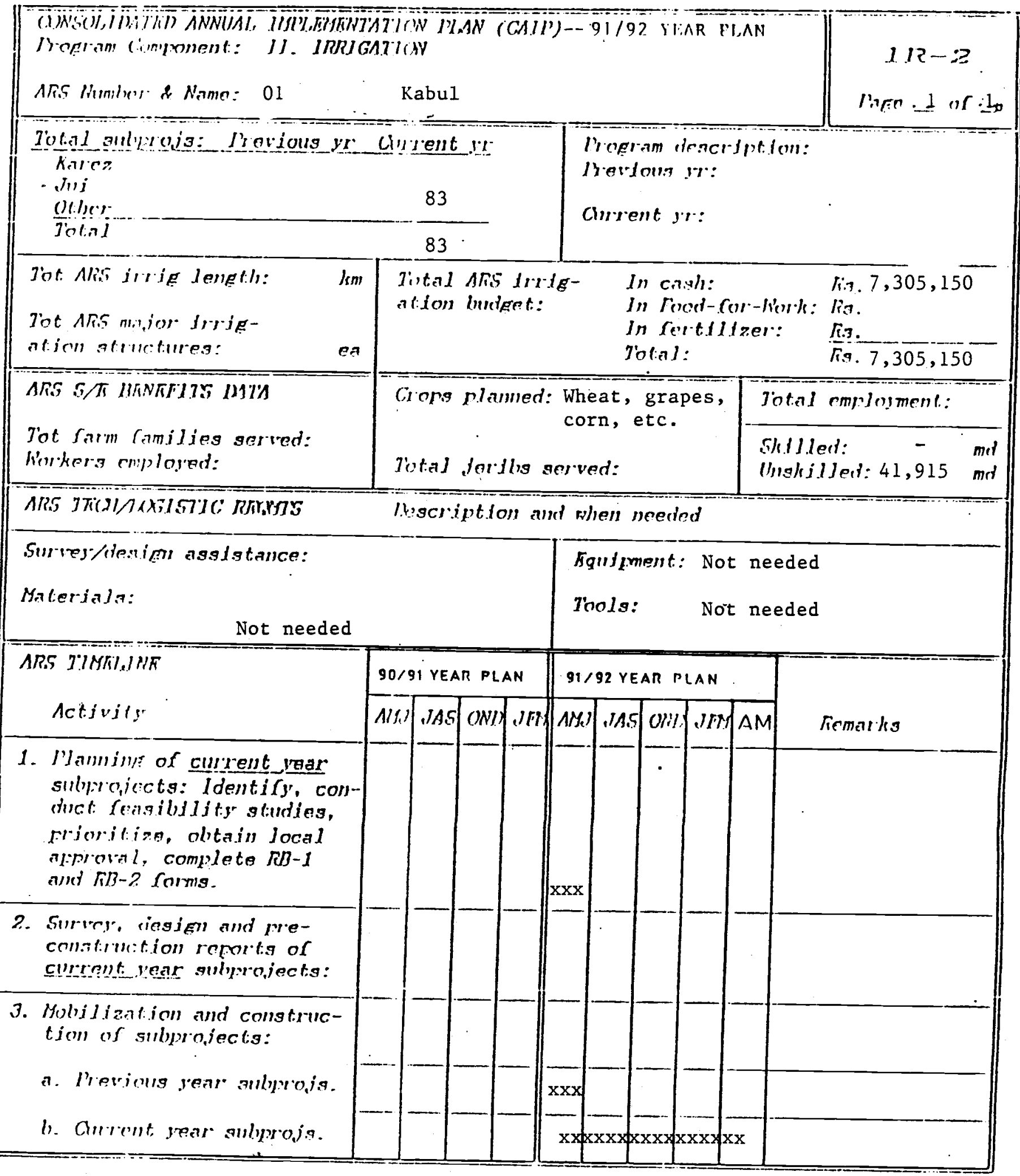




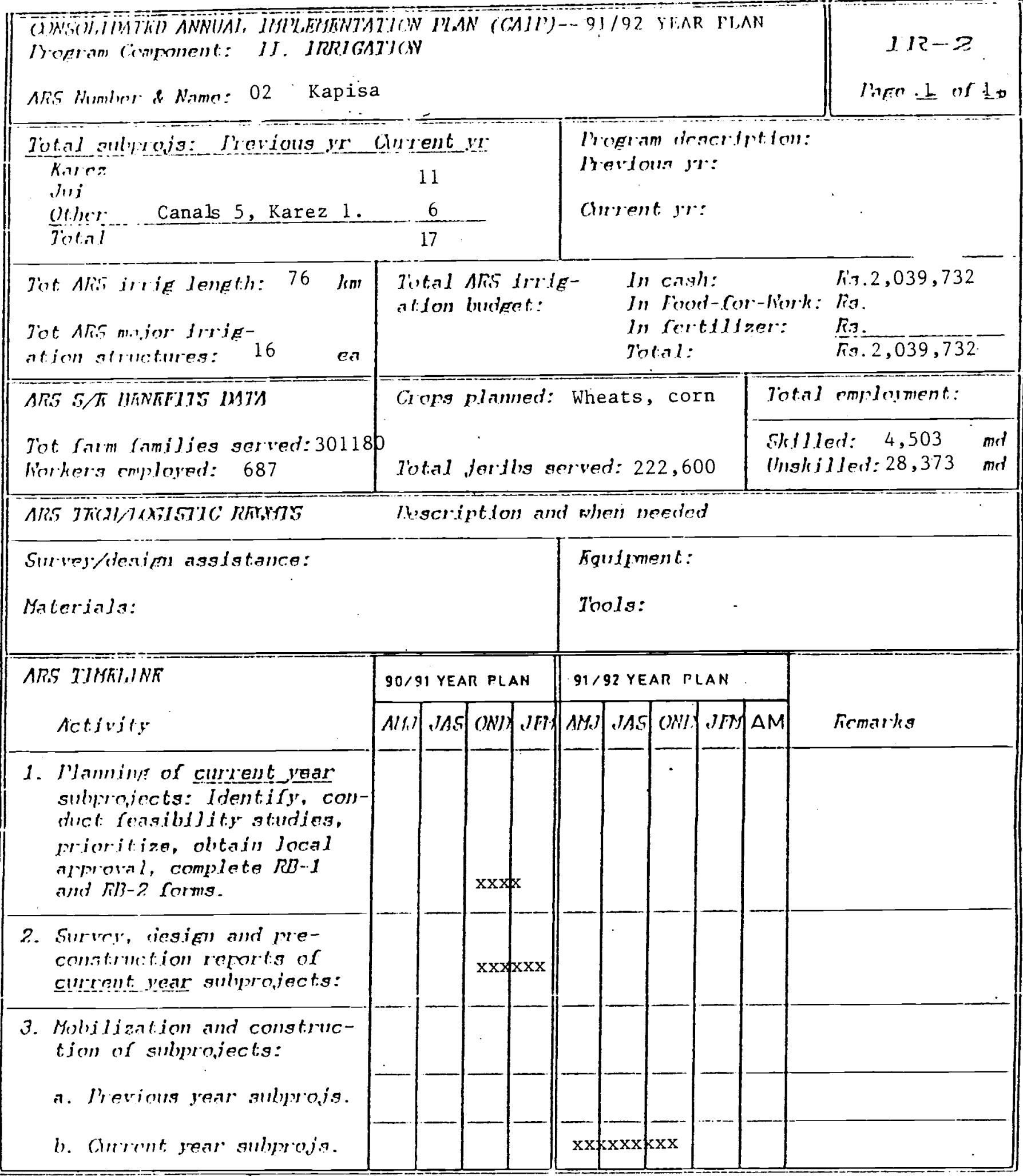




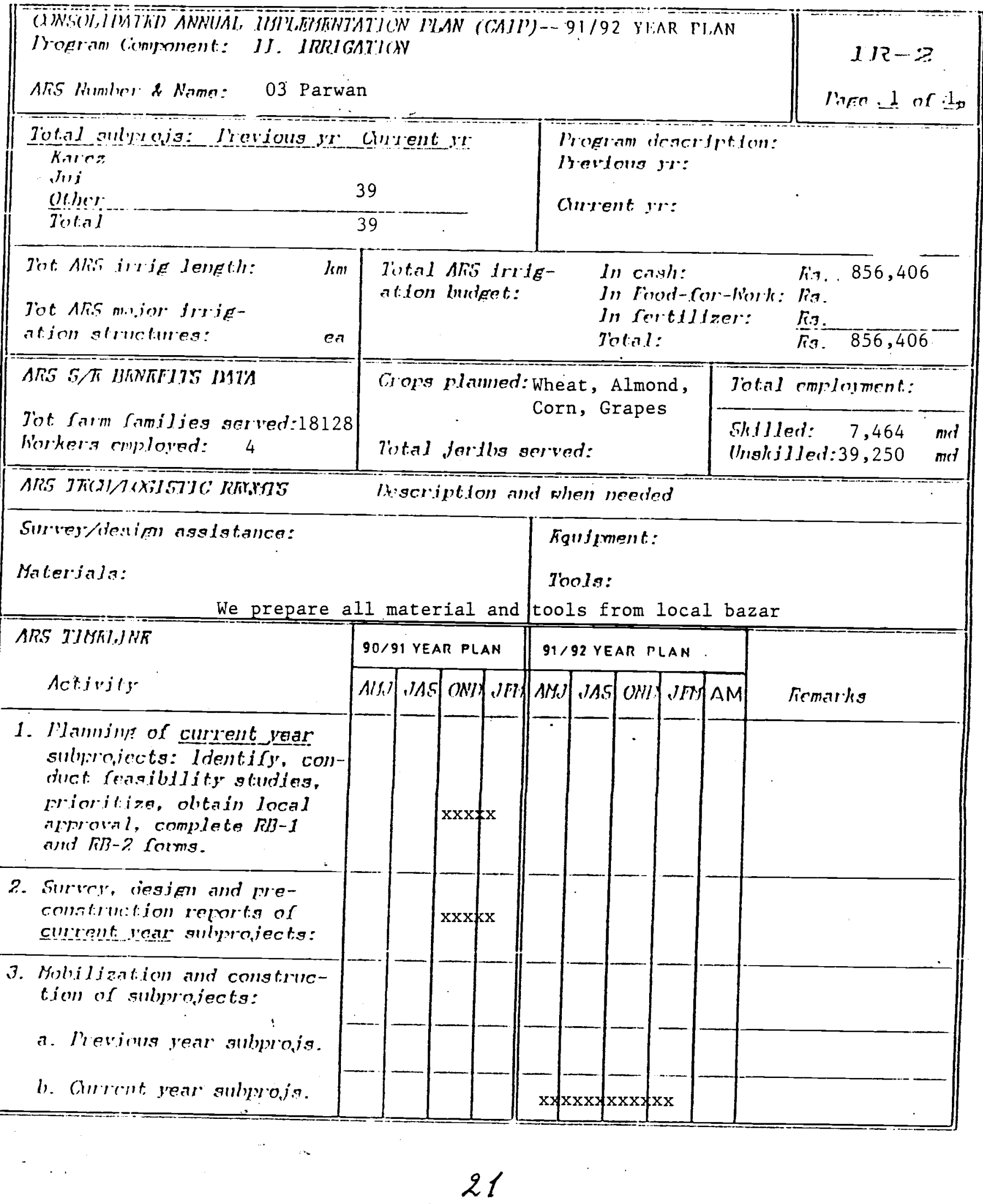




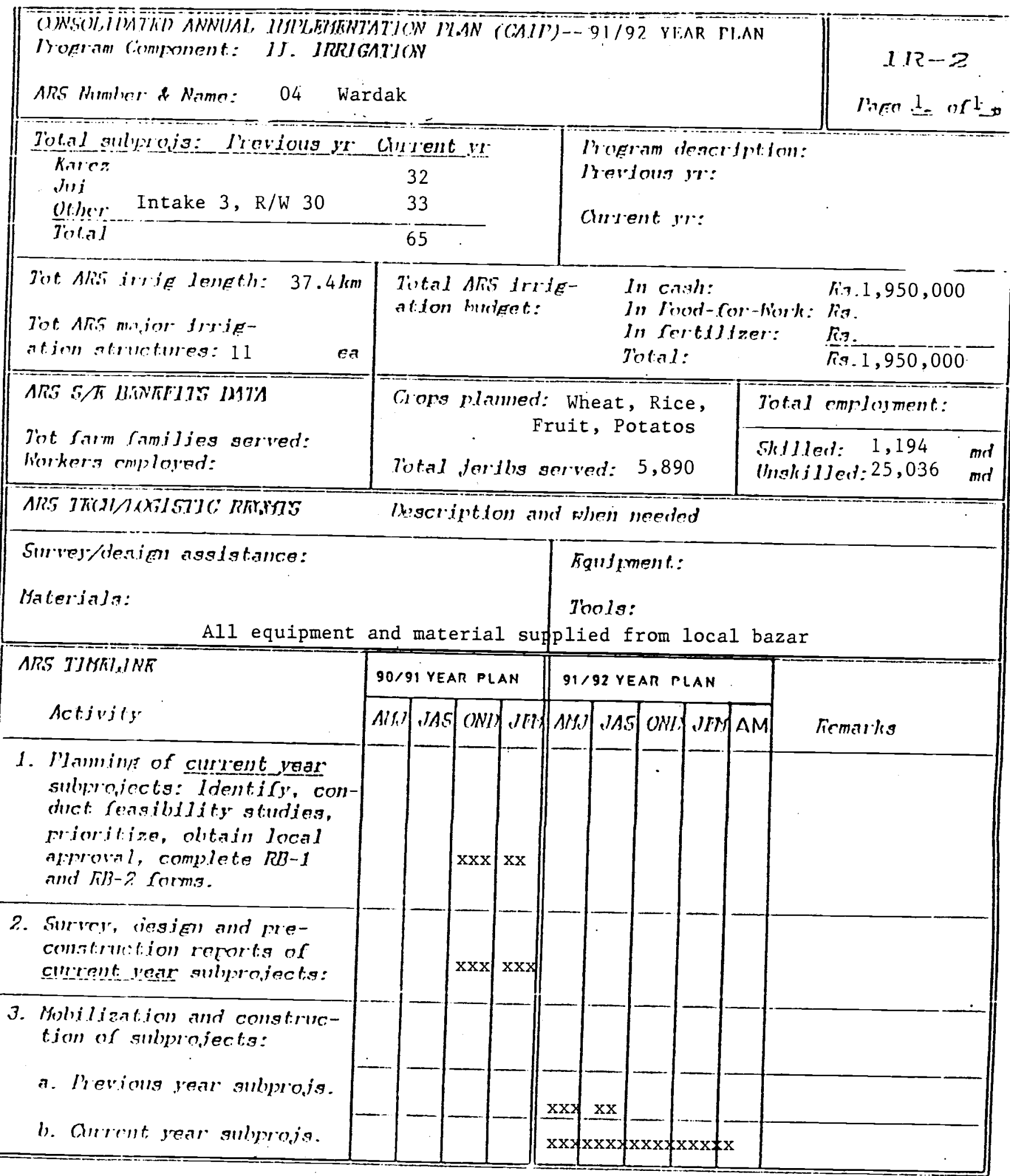

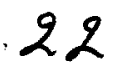




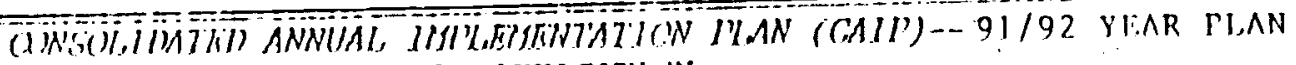

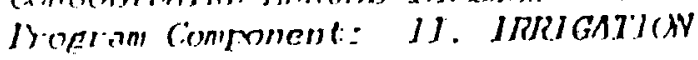

ARS Mumher \& Name: 05 Logar

Iaten

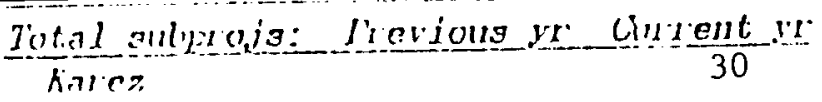

ini

Qtilner.

Dam 1 , Intake $3, \mathrm{R} / \mathrm{W} 5,11$

joini

7it. Alici inig lempth: $0.40 \mathrm{~km}$

Tival Afis irrig- In c.r:Th:

fi. $1,318,897$

Program dicaciption:

lyertong ix:

Ginent yr:

Tot Aris mijor lirig-

aticn atruthes:

ARG $6 / \pi$ UKNTFI7S IMTIA

Jot farm familieg served:

licher:z cmployed:

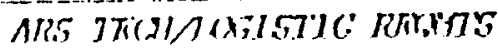

Smrey/ienipon asglstance:

Materiala:
In lood-cor-lines: lia.

In fertilizer: R.?

Jolal:

ation luntegt:

ex

Crops plamed: Wheat, Corn

Total ferilus served: 24,140

Shllled: 2,000 mot

lingkilled: 20,512 mit

Aegcription and when needed

Fquilment:

Thols:

All equipment and tools from local bazar

ARS TJMFIJNK

Activify

1. Maminy of curlent vear sulwoiects: Identify, conduct fenabllity studies. pricritize, obtain local ippoinl, complete $F D-1$ and 18 -? rorms.

2. Surtry, diesign and preconstirutetion reports of clurrent vear sulyrojects:

3. Molitiantion and construetion of subproiects:

a. Mevious year smbrois.

b. Arrent, year sulproja.

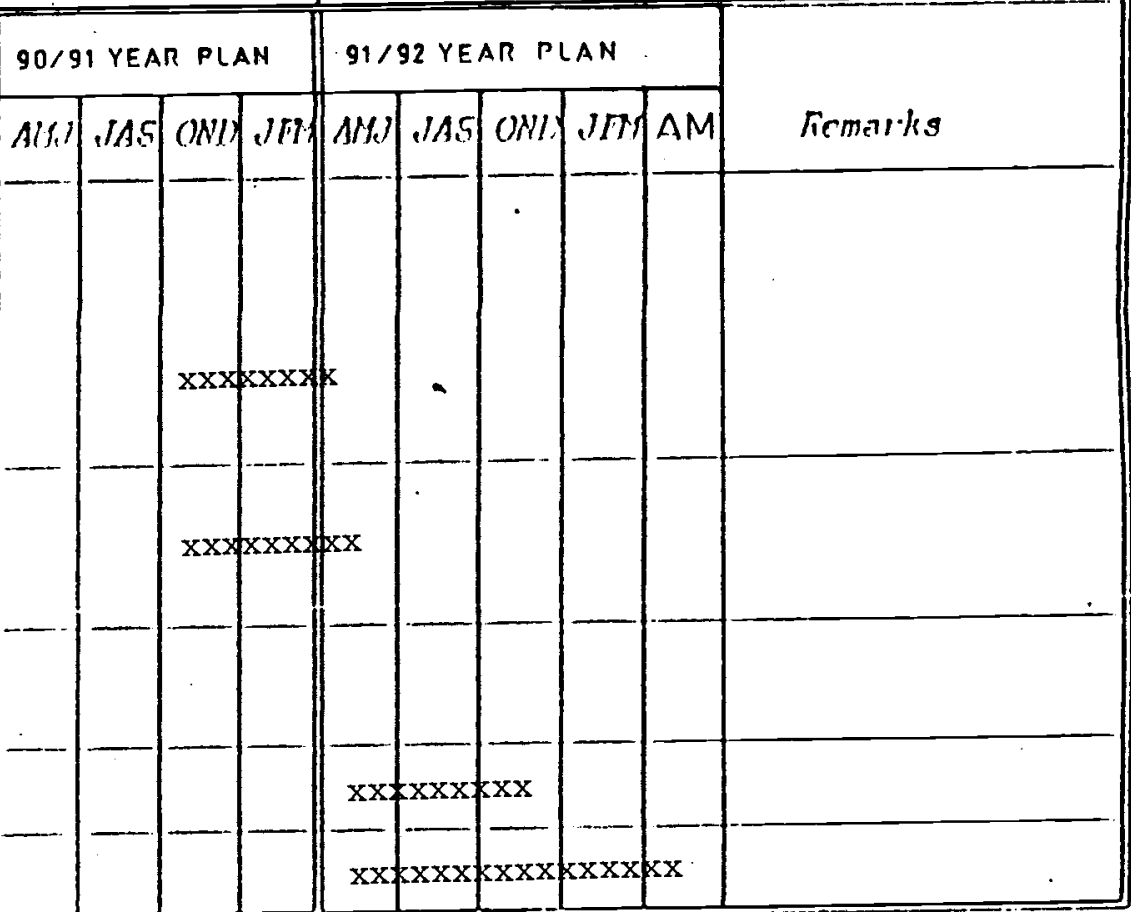




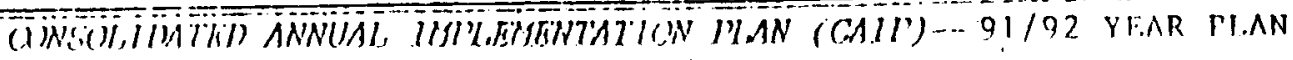

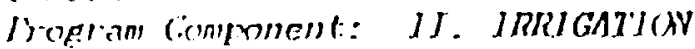

ARS Humbr \& Name: $06^{\circ}$ Ghazni

1 $12-2 ?$

D.

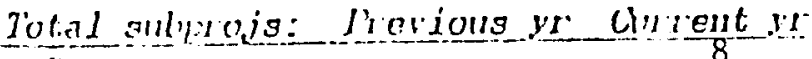

kare?

Mili

9

O) Uer. Intake 2, Canal 1,

inini

5

22

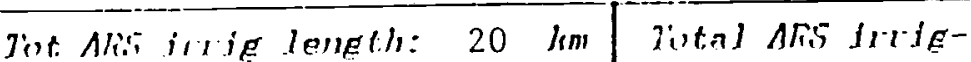
at ton butipet:
The Ars moiner trripation atructures:
21 ea
Proetam description:

liertons y.:

Cinrent yr:

\section{ARG $5 / \pi$ UKNTFITS MIIS}

Jot (arm fomj]jeg served: 23362

linkerg enployed:

272

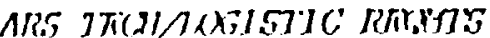

In crish:

fi. $2,183,516$

In lend-ror-link: fia

In rertillzer.

lotal:

R.3.

19. $2, \overline{83}, \overline{516}$

Gopg plamed: Wheat, Fruit, Corn, Potatoes

7otal emrleyment:

Shllled: 22,535 mo

(Ingkilled: 75,370 mir

Surve/ilenipn nsgistance:

kegcription and when needed

Materiala:

Fquifment:

Thola:

Tools and material all from local bazar

ARS TJMAININK

Activilg

1. Hammin!s of clurgent vetar sulyro,icets: Identify, conduct reasibility gtudies, Nioritize, olitain Jocal npworal, complete RB-1 arid rib-? forms.

2. Surury, regign and preconstivition remrlis of cunrente yent sulprojects:

3. Molitiantion and congtruction of subwojects:

a. Lerions rear sulprojs.

b. Curvent yene gulpwoja.

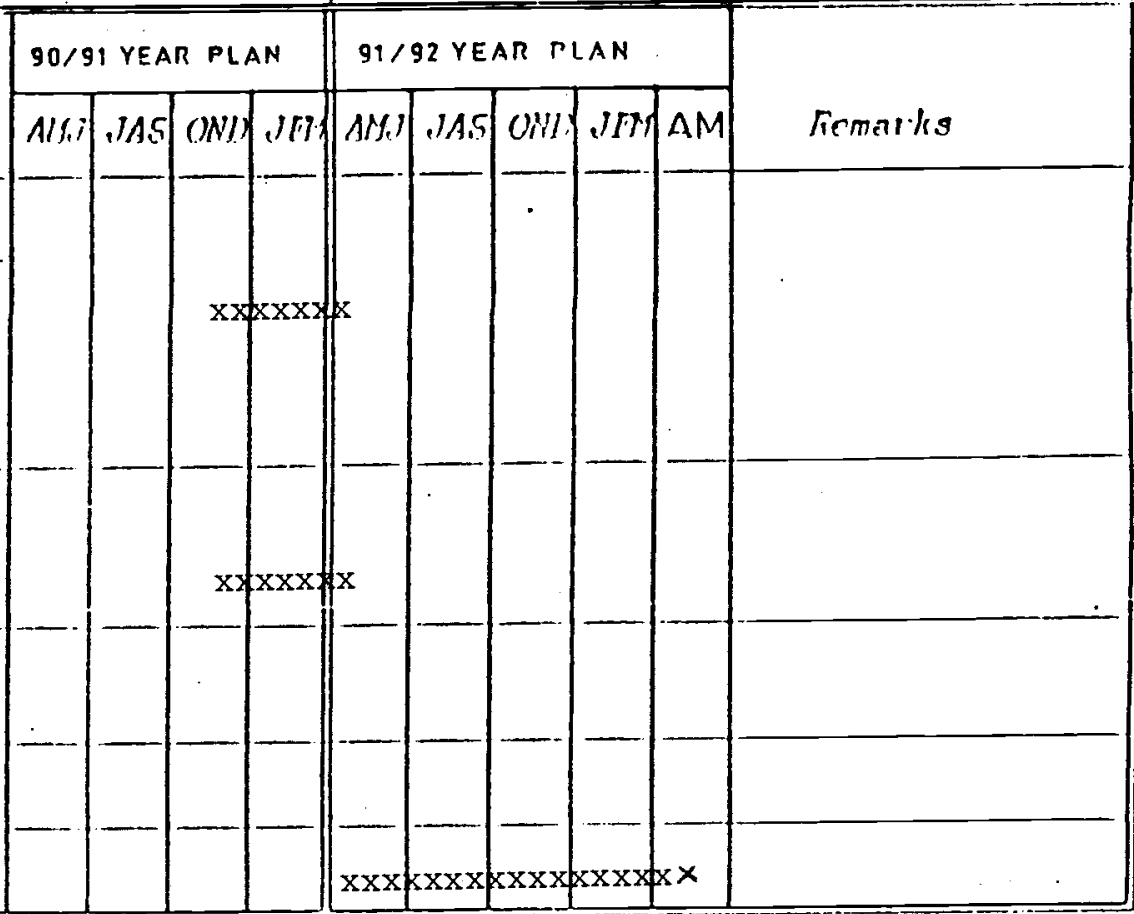




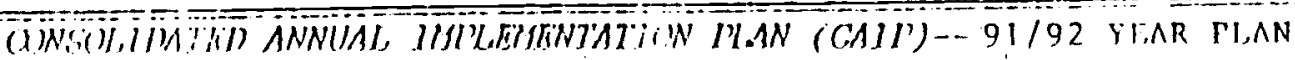

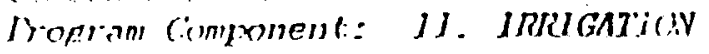

ARS Huminer \& Name:

12 Takhar

l.

Iotol gnlyojg: Irovioug yr clinent yr

Kares

Min

Flume 2, Intake 1 ,

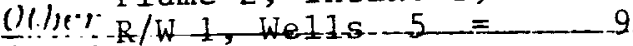

15

\begin{tabular}{|c|c|c|c|c|c|}
\hline 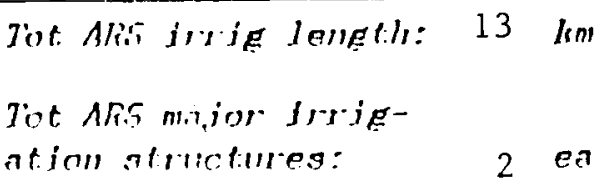 & $\begin{array}{l}\text { Tital ARis frite- } \\
\text { ation buipet: }\end{array}$ & $\begin{array}{l}\text { In congh: } \\
\text { In leod- } \\
\text { In rerti } \\
\text { lofal: }\end{array}$ & fink: & $\begin{array}{l}\operatorname{lig} 1,235,884 \\
\text { lia. } \\
\text { R.3. } \\
\text { Fis. } 1,235,884\end{array}$ & \\
\hline ARS $5 / \pi$ UIKNKF17. 11171 & Clops plammed: Wheat & $\therefore$ Corn & Iotal & emplesment: & \\
\hline $\begin{array}{l}\text { Jot farm families served: } 2190 \\
\text { linkerg culloged: } 634\end{array}$ & Jotal forllug sorved & $=1,790$ & $\begin{array}{l}\text { Shllle } \\
\text { Wnakit }\end{array}$ & $\begin{array}{l}\text { ed: } \quad 25 \\
\text { lled: } \\
31,042\end{array}$ & $\operatorname{mrd}$ \\
\hline
\end{tabular}

Smrey/denim asststance:

Fquiment:

Maleriala:

Toola:

All material and equipment supplied from local bazar

ARS TIIMGI,INK

Activity

1. Diaminily of curryent vear sulpoiects: ldentiry, conduct reasibility gtudieg, Frioritize, olitain Jocal arproinl, complete RB-1 and $K B-2$ forms.

2. Surver, iesign and preconstirlution reprotis of cllyrent year sulproiects:

3. Molifilization and construction of sulpro,jects:

a. Previons year ankpois.

b. Amrent year sulperia.

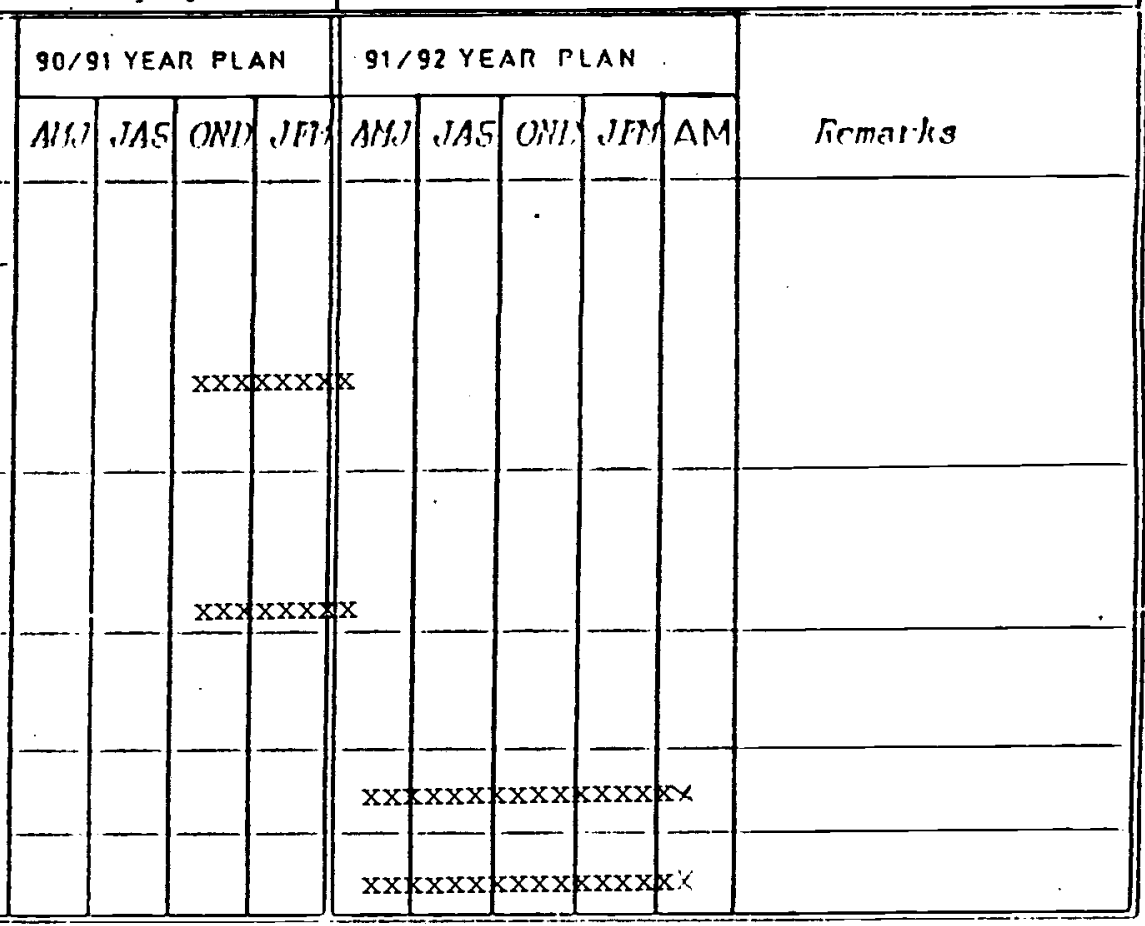




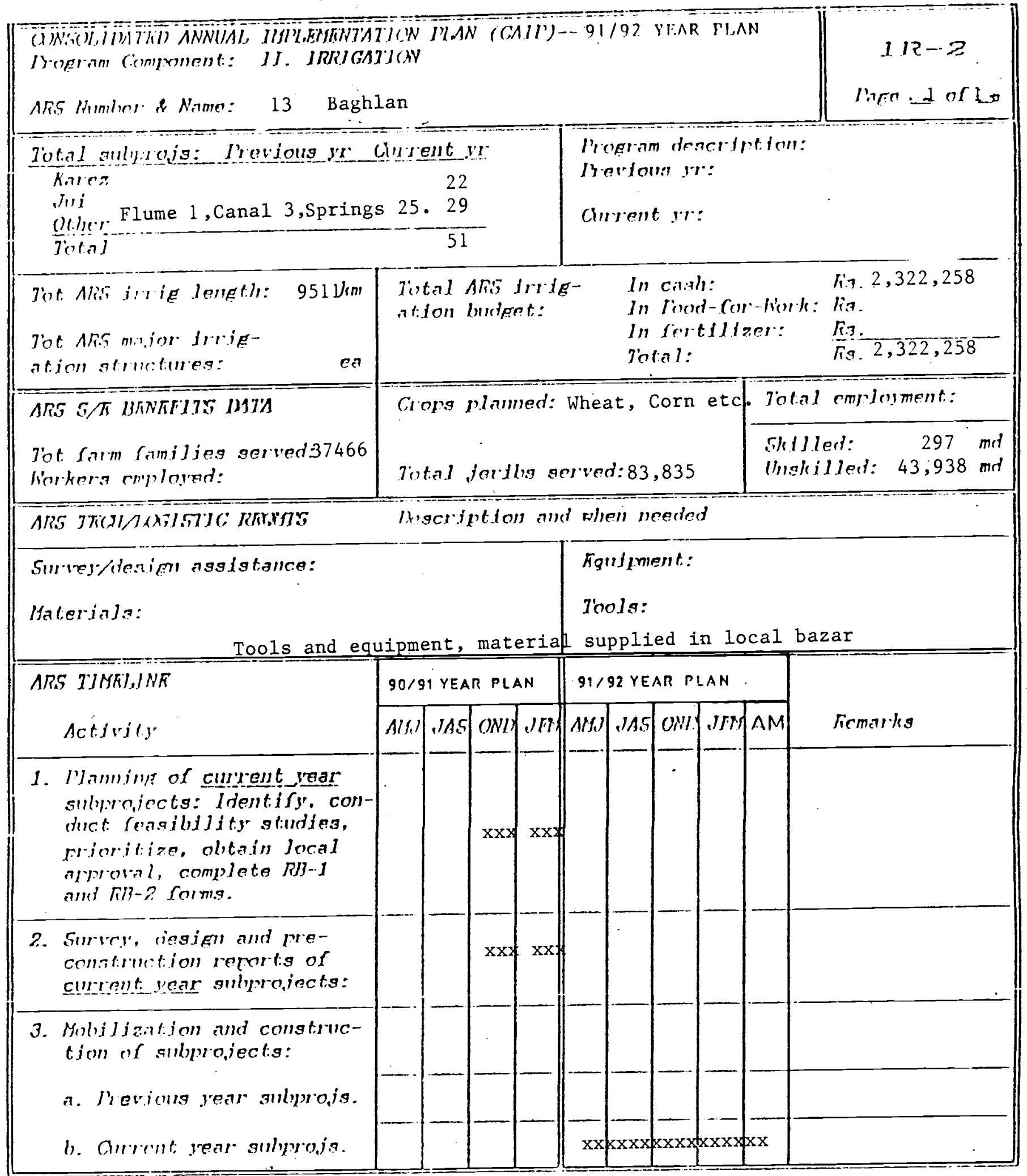




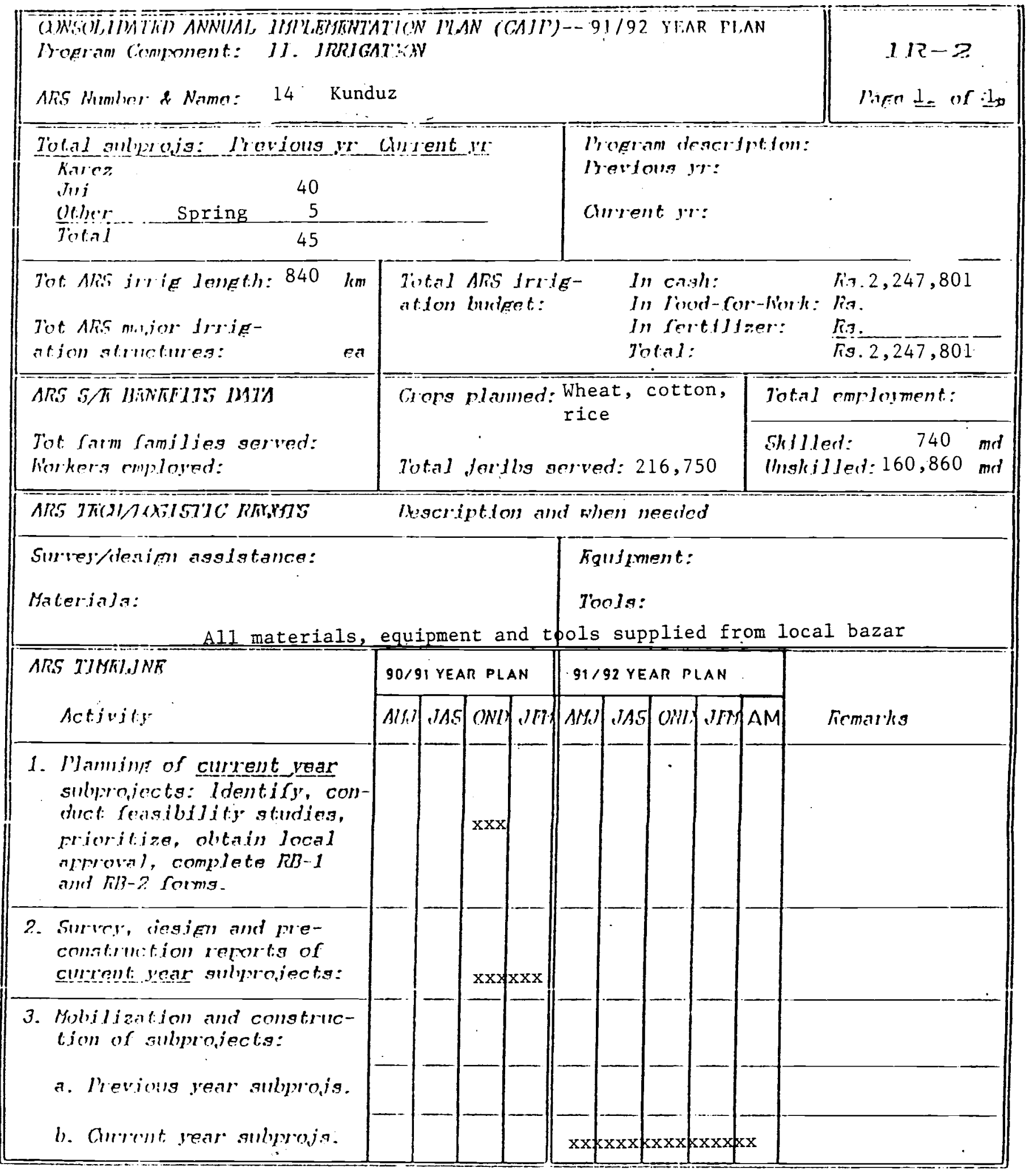




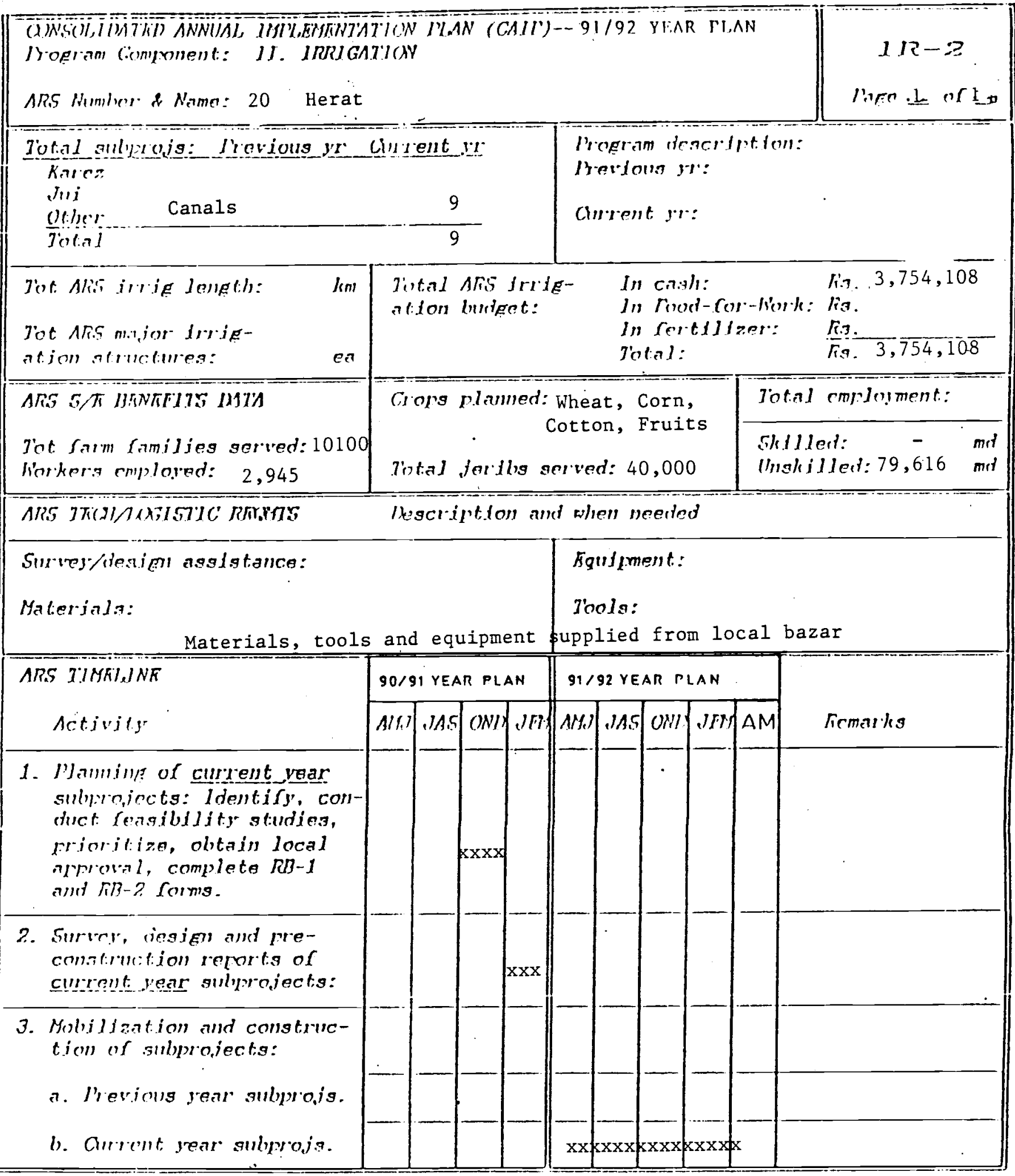




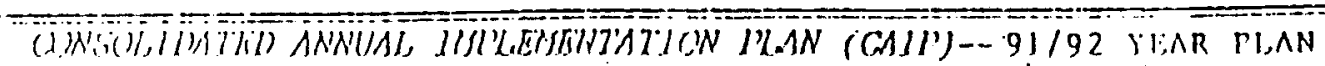

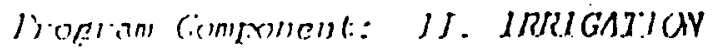

Mris liumlen \& Name: $24^{\circ}$ Kandahar

limen of of 10

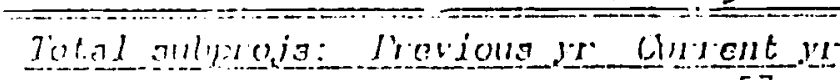

sine?

57

I1)i Flume 1, Dam 1,

Ot) Intake 1, Irish Bridge 1.

Joitial

4

61

linegram reacthtilon:

lieveloti:s yn:

Chrent yo

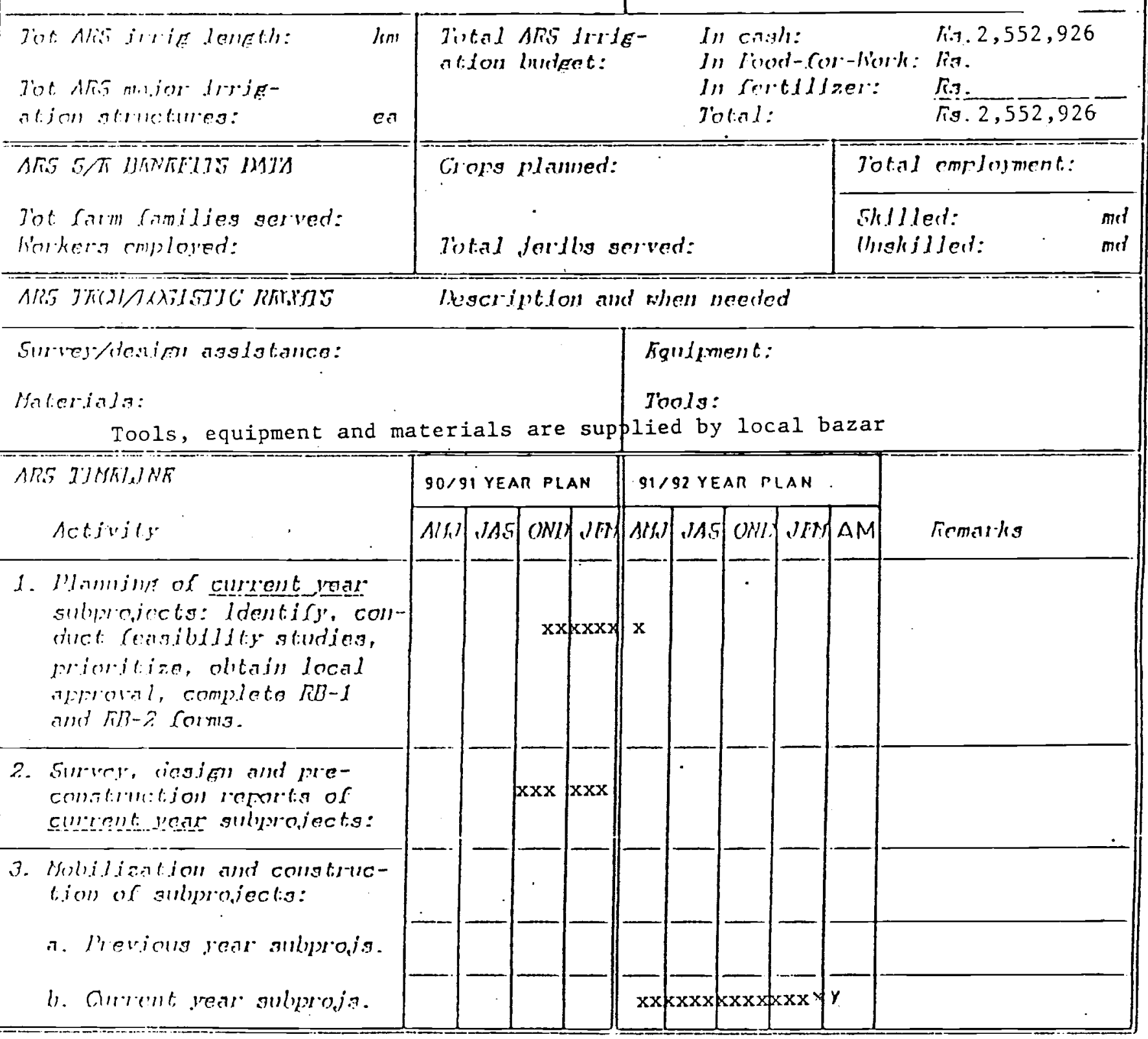




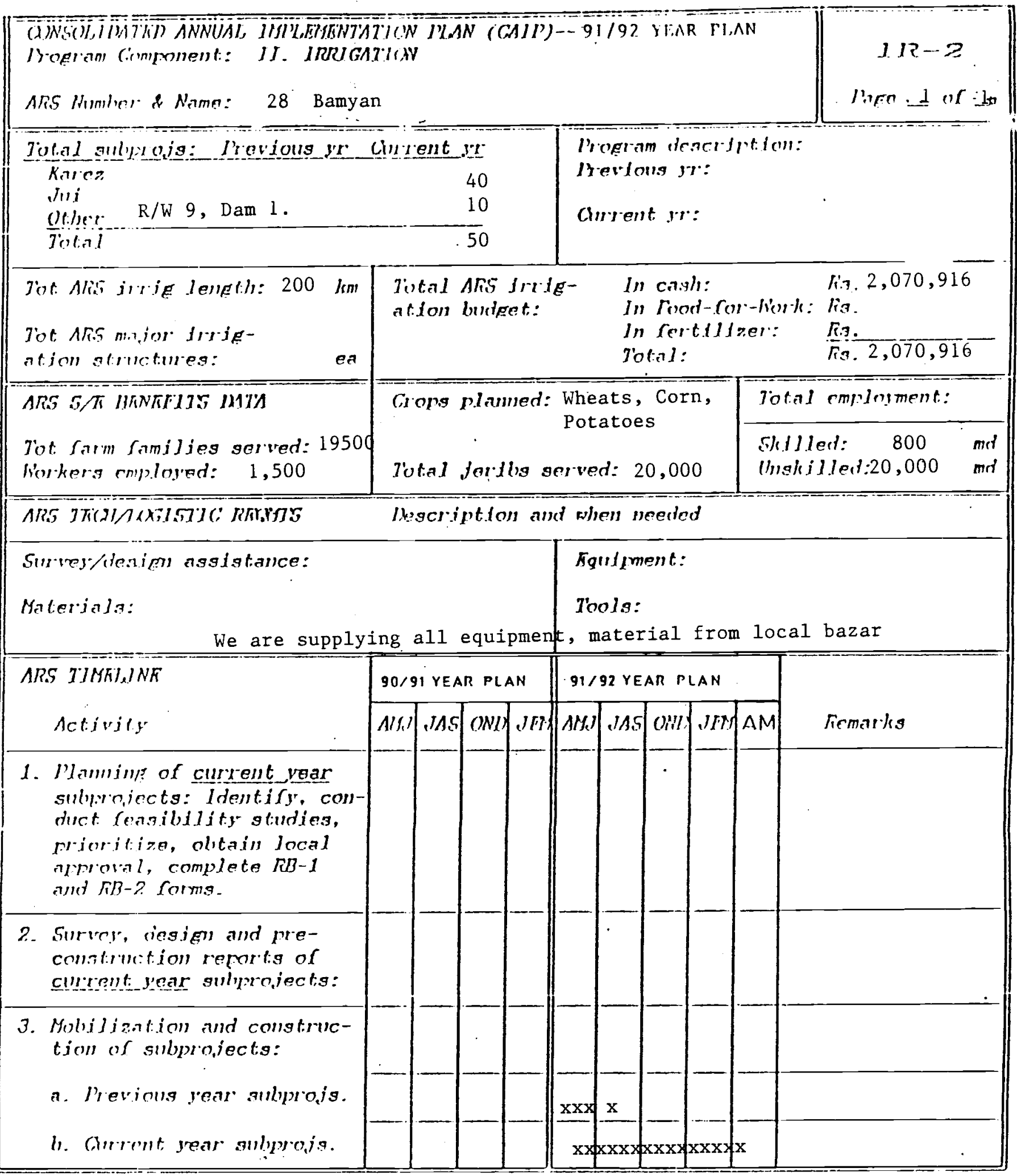




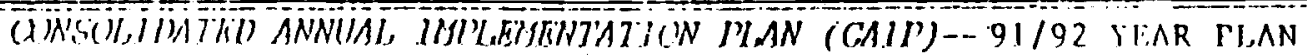

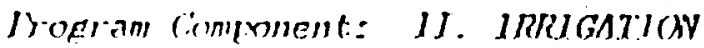

$112-2$

$\operatorname{laren} 1$ ort

Ahs Humler \& Name: 28 Paktika

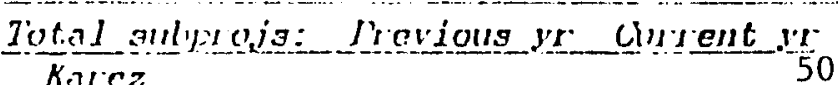

$.11 \mathrm{~F} 2, \mathrm{D} 5, \mathrm{R} / \mathrm{W} 3, \mathrm{IB} 2$, IW 8 , (l)er Spr 30 , Irish Bridge 1 . Ji, ini lingram deactiptidu:

Herlous yr:

Grrent, yr:
Iot Alit irrig length: $382 \mathrm{lsm}$

Tot AR. mirior thrig-

ation afinctures:

ARG $6 / \pi$ HINKFITS MUTS

Tht farm Camilieg served:

linkera riployed: 50,057 lival ARS ITHEation butget:
In c.n:3/1:

In Fout-cor-lierk: ra.

In fertlitizer:

Iolal:

R.3.

$1 0 \longdiv { 2 , 4 } \overline { 5 3 , 2 0 8 }$

fi. $.2,453,208$
Clopg plammed: Wheat, Corn, Potatoes, Barley

Total forllig served: 33,904 7otal employment:

Fillled: $6,530 \mathrm{mr}$
(hiskilled: 43,527 mi

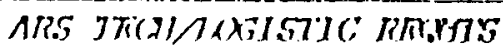

lescription and when needed

Shrej/icaim asslgtance:

Materialo:

Fquipment:

Thols:

Materials, equipment and tools afe supplied from local bazar

ARS TJMFINAK

Activity

1. Mimmis!s of current vear. subroiects: Irentiry, con duct feagibldity studies, rioritize, olitain local

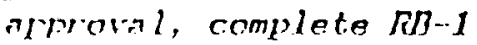
הII 5 B-? forms.

2. Suruer, iegign and preconstirvetion reports of current vare gulproiects:

3. Moliditation and construction of subproiects:

a. Mevinug year sulyroig.

b. Aurent, year sulpyoja.

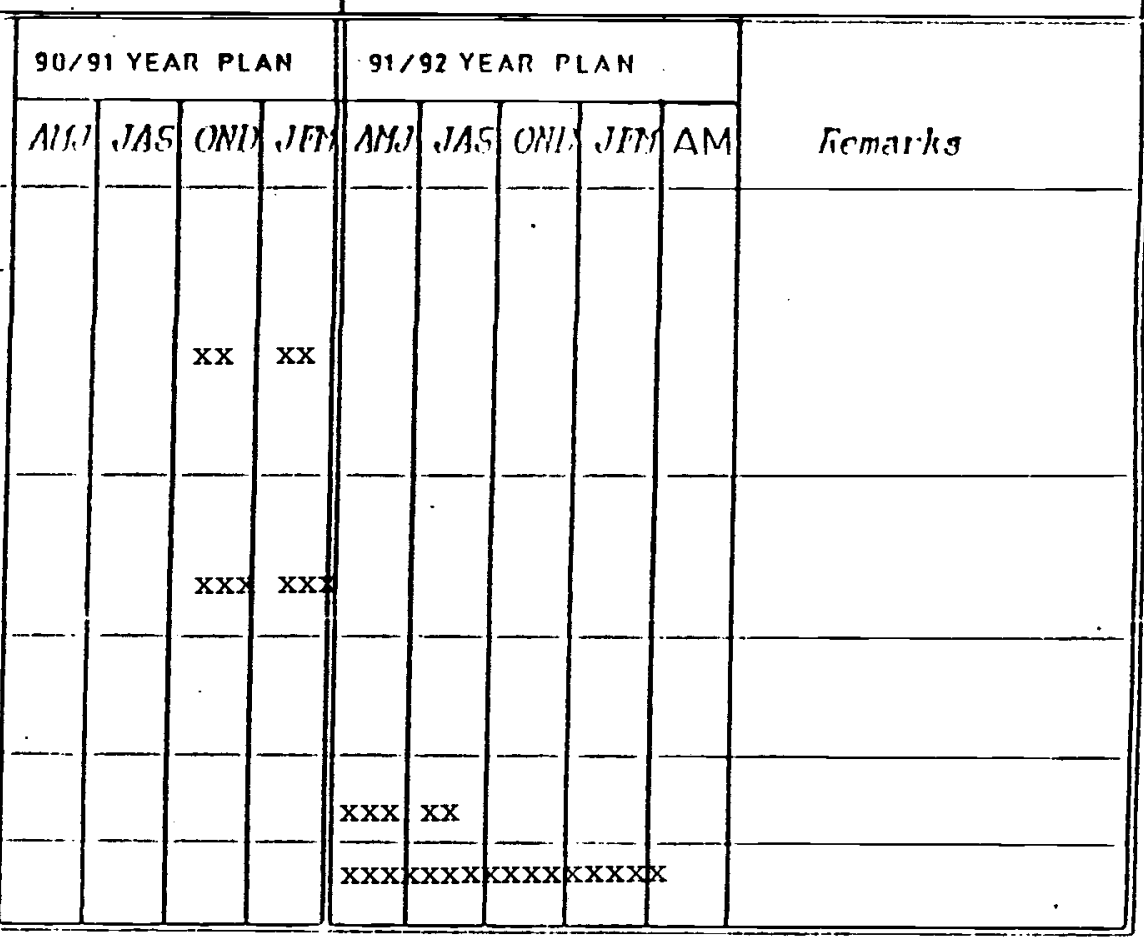




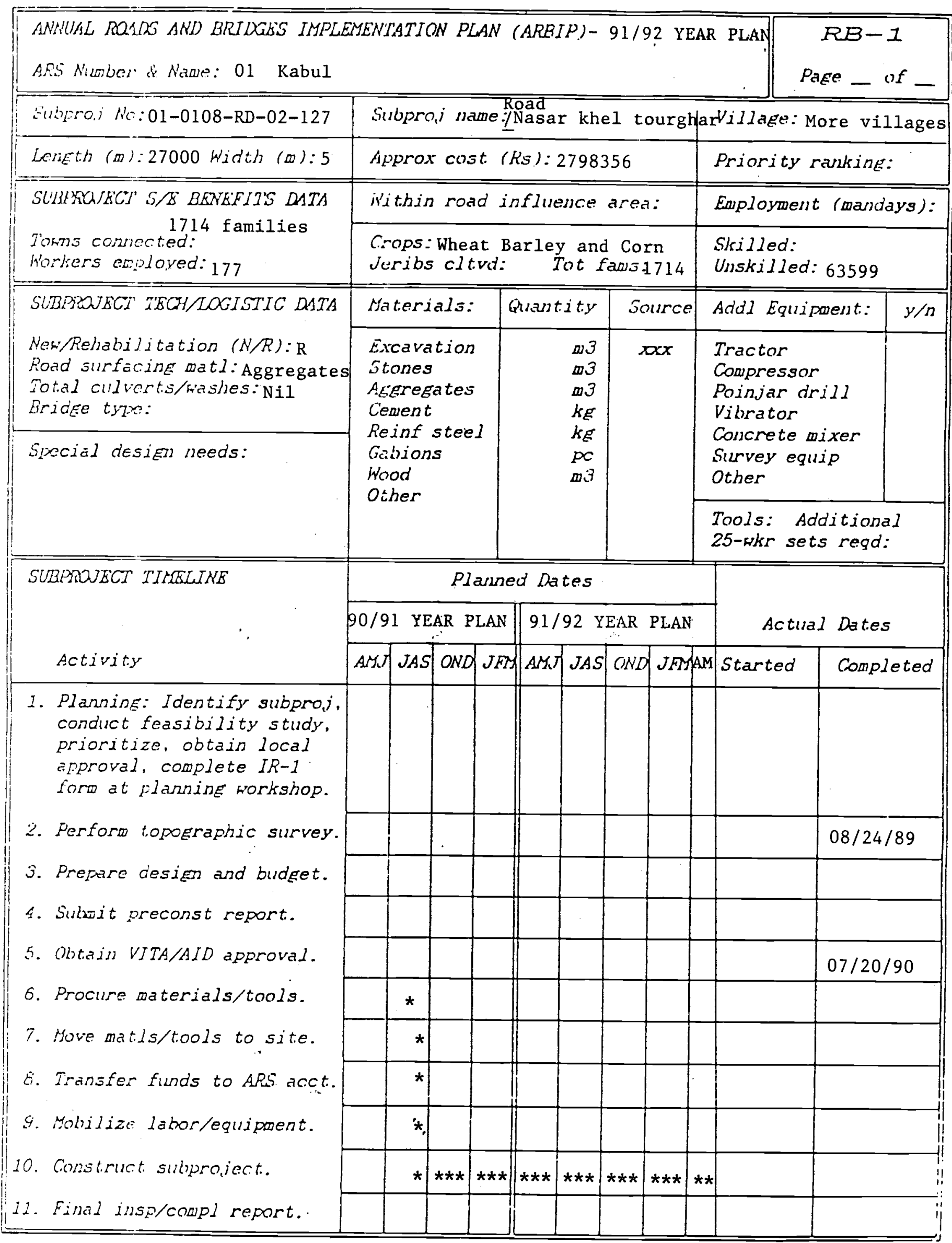




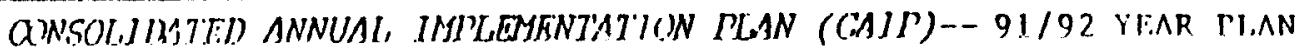

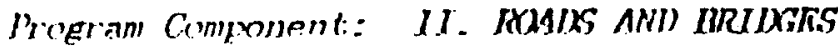

ARS Number: 01

ARS Name: Kabul

I'ike 1 of 1

Tot coad lengt:l) pimet: km

ARS ST WKNEFITS MMTA

(n)

Total bridgeg plamned: 1

RH Lnd (R.9): 414,875

No of torim commected:2 villagestotal farm tamilies:

Workers employed: 264

Tolal Terils:

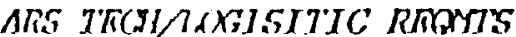

Nescription

Design Assistant is needed

Materials:

Fquipment:

Thols:

Tools and materials supplied from local

ARS TIMKIJINK

Activity

1. Plaming: Identify subprojects, conduct reasibility studies, prioritize, obtain local approtal, complete IR-1 and IR-2 forms.

2. Survey, design and proconatruction reports for subproject by priority.

Highest ranking $1 / 4 t h$ :

Next ranking 1/4th:

Next. rankjug 1/4th:

Lowes: ranking 1/4th:

3. Mobiliantion and construction of subprojects by priorily.

Hjghest: ramking 1/4th:

Next: 1rubing 1/4th:

Next ranking 1/4th:

lowegt ranking 1/4th:

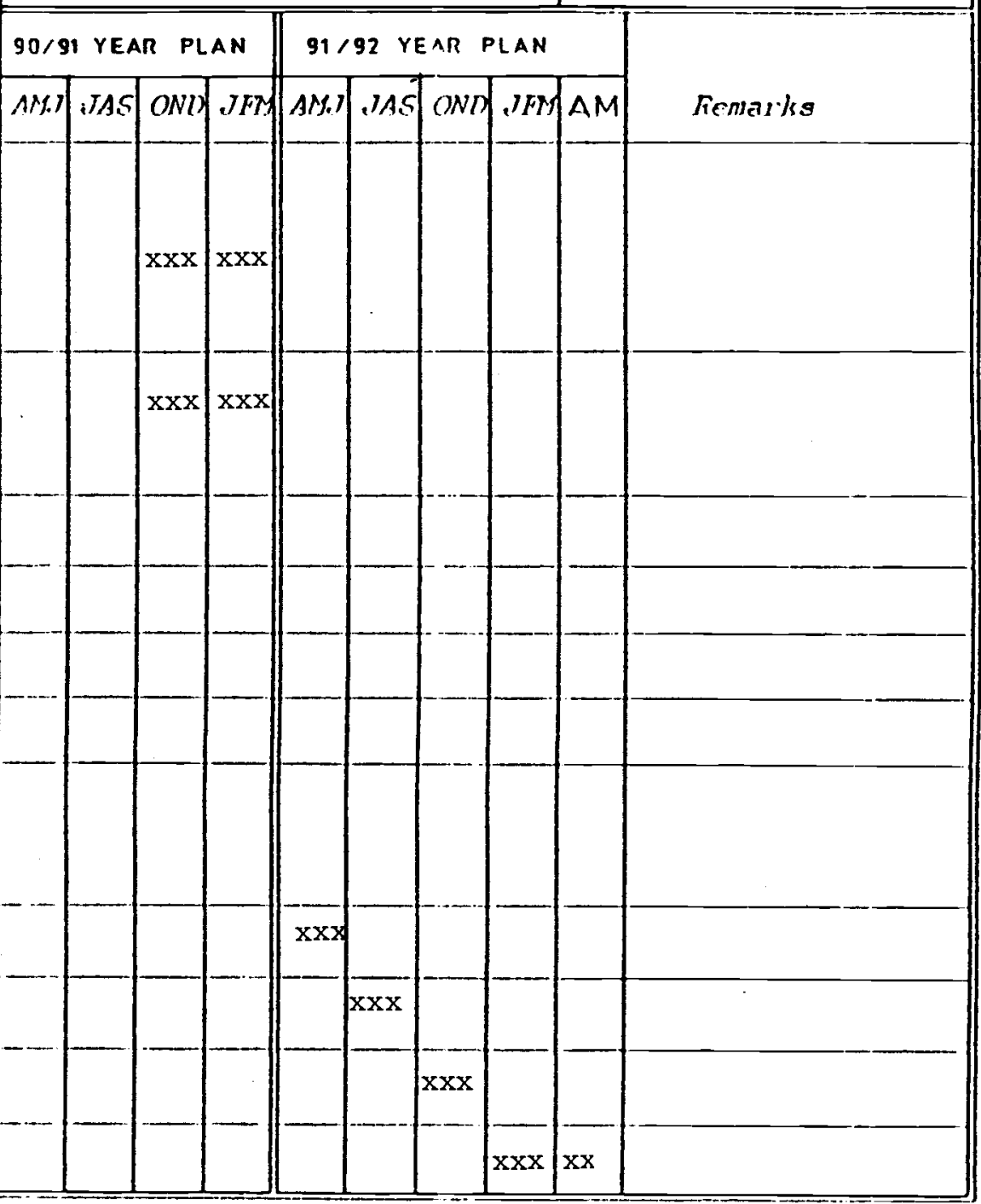




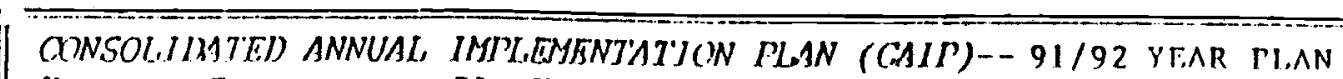

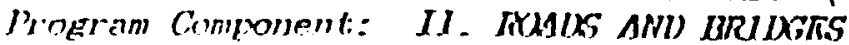

ATS Number: 02

ARS Name: Kapisa

Irike 1 or 1

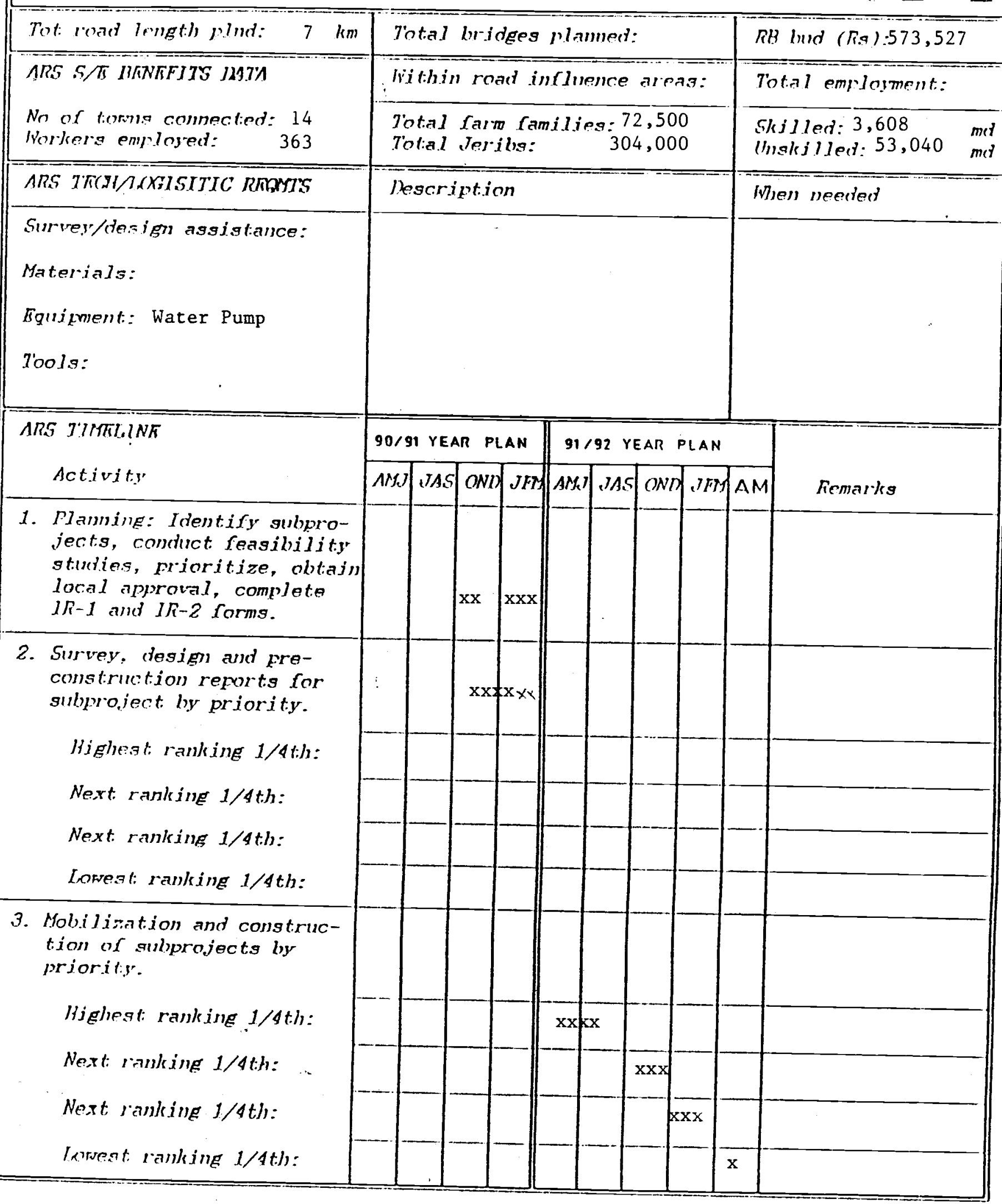


AfG Name: Parwan

Irike 1 of 1

Tot roat length find: 39 lin

Total bridges plamed:

RB lud (R.a) 1,095,053

ARS $5 / \pi$ BKINEFITS IMTA

No of towns comnected: 14

Workers employred:

ARS TK(JIRAXISITIC RMAMTS

Survey/design assistance:

Materials: Roller, Tractor

Bquikment:

Tools:

We supply all material and tools from lodal bazar

\section{ARS TITMRI.INK}

Activity

1. Plaming: Identify gubprojects, conduct reasibility studies, frioritize, obtain local approval, complete $1 R-1$ and $I R-2$ forms.

2. Survey, design and preconstruction reports for subproject by priority.

Highegt: ranking 1/sth:

Next ranking 1/4th:

Next ranking $1 / \phi t h$ :

Lowe? : ranking i/4th:

3. Mobilization and congtruction ar arshprojects ly priorily.

Highest: ranking 1/4th:

Next ranking 1/4th:

Next ranking $1 / 4 t h$ :

Leweal: ranking 1/sth:

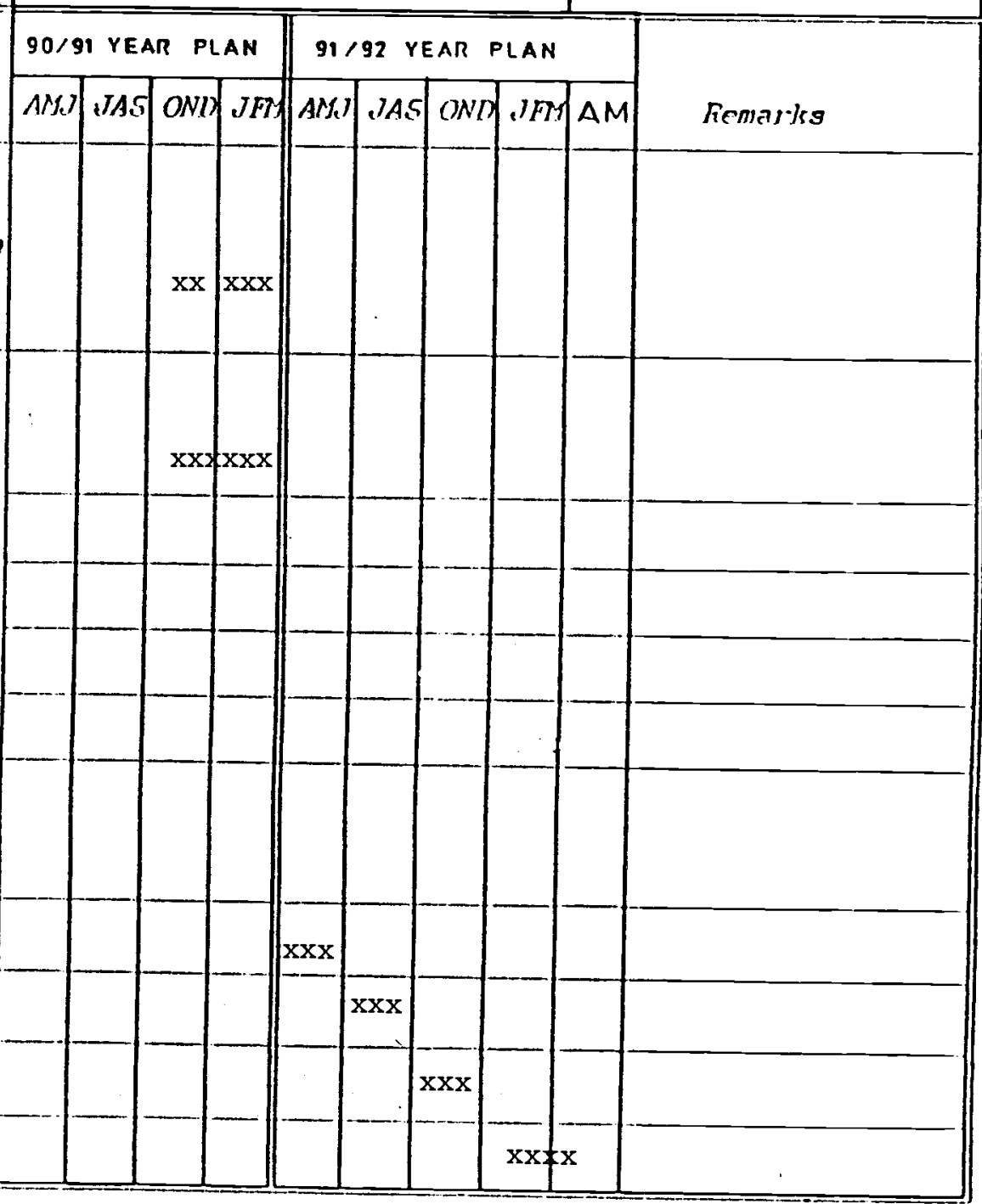


ARS Name: Wardak

I'TEe 1 of 1

\begin{tabular}{|c|c|c|c|}
\hline Tot road length plud: $\mathrm{km}$ & Total bridges plamned: 15 & \multicolumn{2}{|c|}{$R B$ lnd (Ra) $=450,000$} \\
\hline ARS $S / \pi$ WRNKFITS DMTH & h'jthin road influence areas: & Total emplc & \\
\hline $\begin{array}{l}\text { No of tomma comnectied: } \\
\text { ivorkers emplared: }\end{array}$ & $\begin{array}{l}\text { Total farm ramilies: } \\
\text { Total Teribs: }\end{array}$ & $\begin{array}{l}\text { Skilled: } \\
\text { Inakilled: }\end{array}$ & $\begin{array}{l}m e d \\
m e d\end{array}$ \\
\hline
\end{tabular}

Surver/design asgistance:

Materials:

Equipment:

Jools:

Tools and equipment supplied from local pazar

ARS TIMETIINK

Activit.r

1. Plaming: Identify subprojects, conduct feagibility studies, frioritize, obtain local approval, complete $1 R-1$ and $I R-2$ forms.

2. Survey. degign aud preconstruction reports for subproject by prjority.

Highesgt ranking 1/4t/s:

Next ranking $1 / 4$ th:

Next ranking 1/4th:

Lorvest. ranking $1 / 4$ th:

3. Mobilization and construction of subprojects by priority.

Highest: ranking 1/4th:

Next: ranking 1/4th:

Next ranking 1/4th:

larvegt: ranking 1/4t\}:

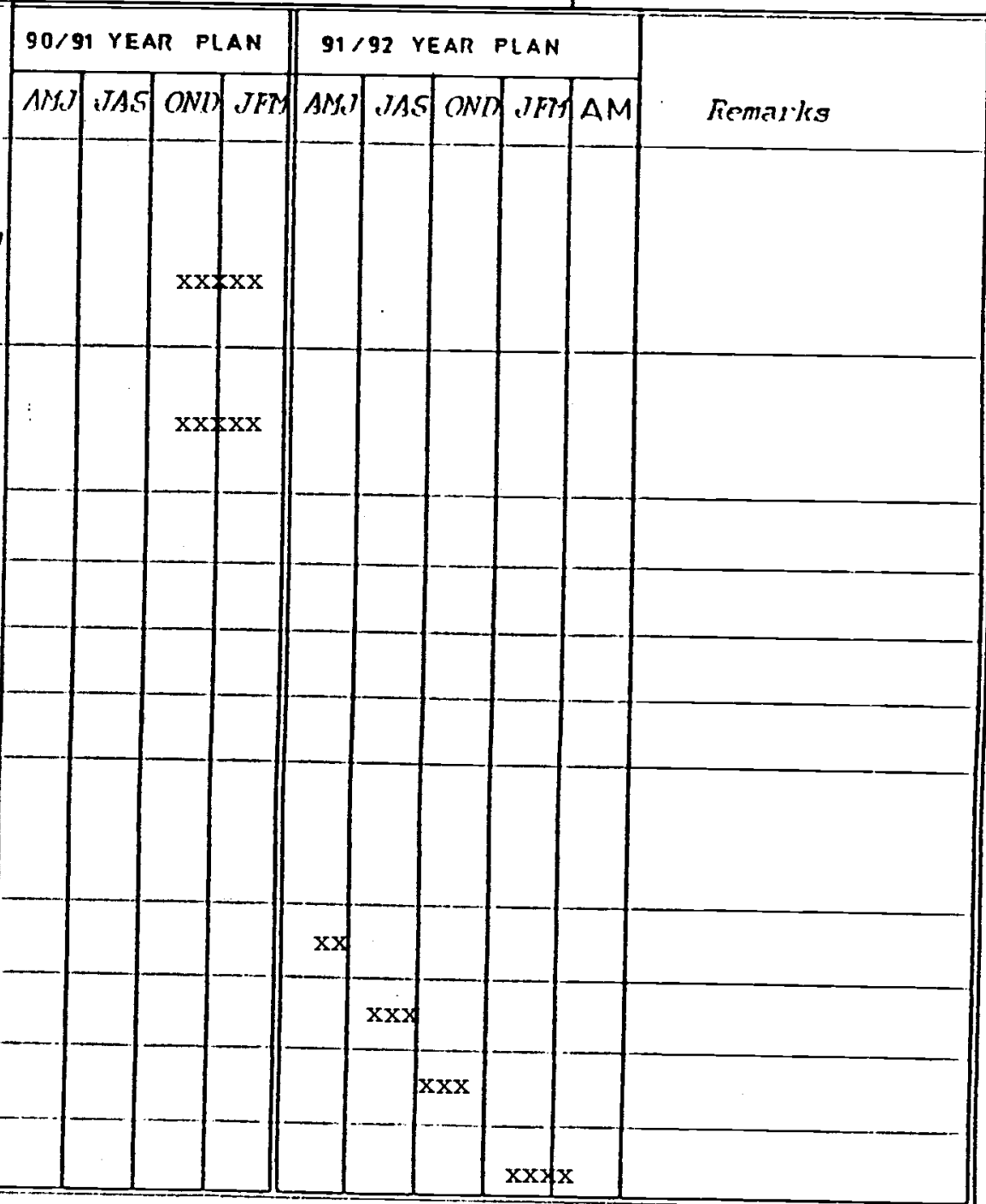


OONSOLIIXITTID ANNUAL, IMITIOMFNTATICN PLAN (CAIP)-- 91/92 YFAR PI.AN

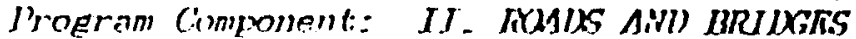

ATS Number: 05

ARS Name: Logar

Irige 1 of 1

Tot: roat Jength find: $20 \mathrm{~km}$ Total bridges plamed: 2

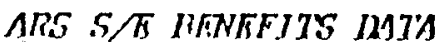

No of torma comnected: 150

Worjera employed:

lotal ram lamilies:

Total Teribs: 8,758

ARG TRII/ROXISITIC RHAMTS

Survey/design asgistance:

Materials:

Equiprient:

7'ools:

All tools and material supplied from local bazar

ARS TIMKI.INK

Activit:

1. Plaming: Ietentify subprojects, conduct feasibility studies, prioritize, obtain local ipproval, complete $1 R-1$ and $I R-2$ forms.

2. Survey, desien and preconstruction reports for subproject ly priority.

Higheas ranking 1/4t:h:

Next ranking 1/4th:

Next ranking 1/4th:

lowest: ranking l/4th:

3. Mobilization and construction of subprojects ly priorityr.

Highest ranking 1/4th:

Next: ranking 1/4th:

Next ranking 1/4th:

lakregt ranking 1/Atb:

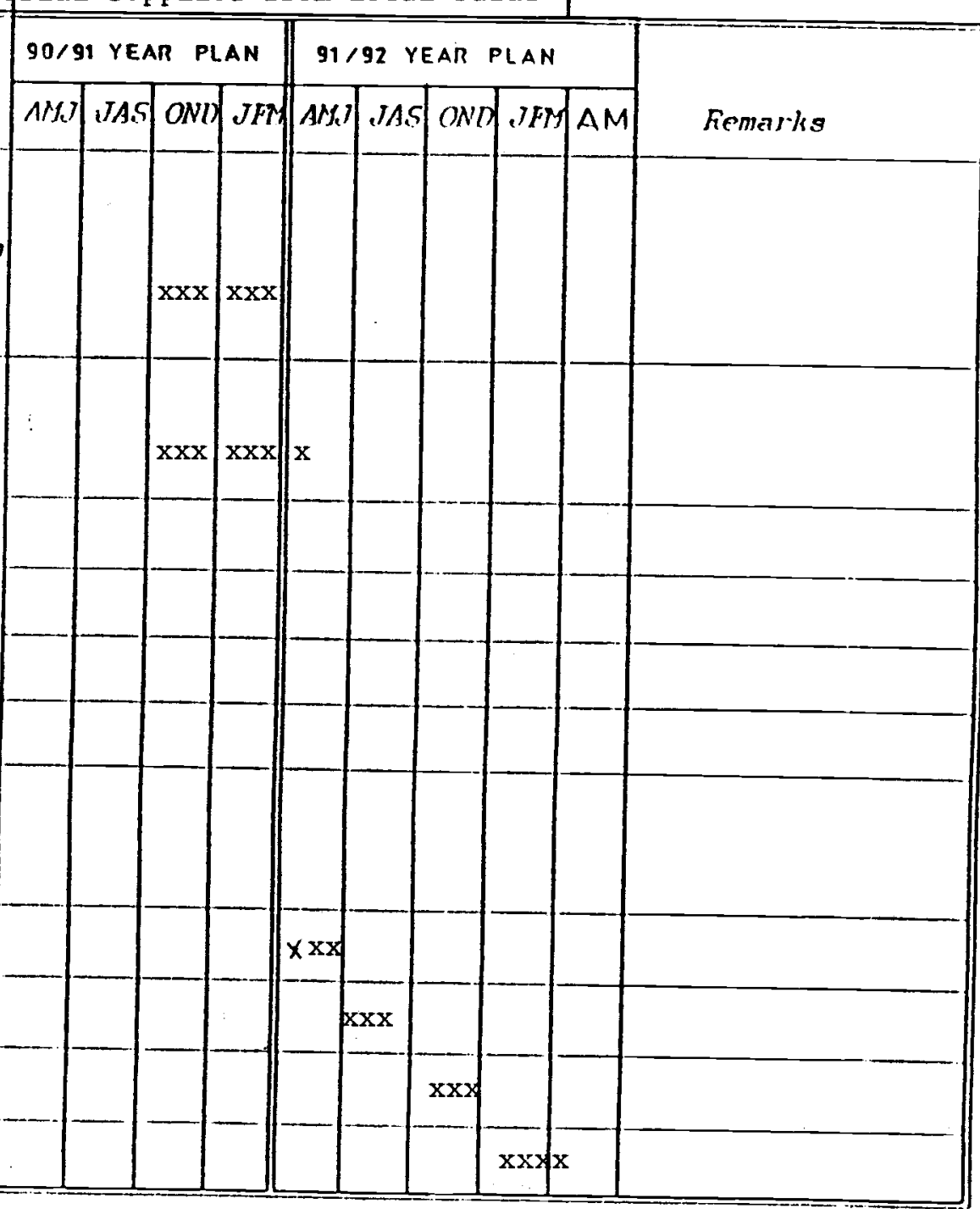


ARS Nimber: 06

ARs Name:

Ghazni

Irike 1 of 1

Tot: road length plind: $\mathrm{km}$

ARS $5, \pi$ UKNTFITS IMTA

No of toms connected: 9

Workers emloyed:

ARS TRIIIAXYISITTC RMAYTS

Survey/design agsistance:

Materials:

Equizment:

Tools:

All tools and equipment from local bazar

ARS TIITKI.INK

Activity

1. Plamming: Identify subprojects, conduct feasibility studies, prioritize, obtain local approval, complete $I R-1$ and $J R-2$ rorma.

2. Survey, design and preconstruction reports for subproiect by priority.

Highegt ranking 1/\$t: :

Next ranking 1/4th:

Next: ranking $1 / d t h$ :

Loregt ranking 1/4th:

3. Mobilization and construction of subprojects by priority.

Highest: ranking 1/4th:

Next: rronking 1/\$t:l:

Next ranking 1/4th:

lowegt ranlsing $1 / 4$ th :

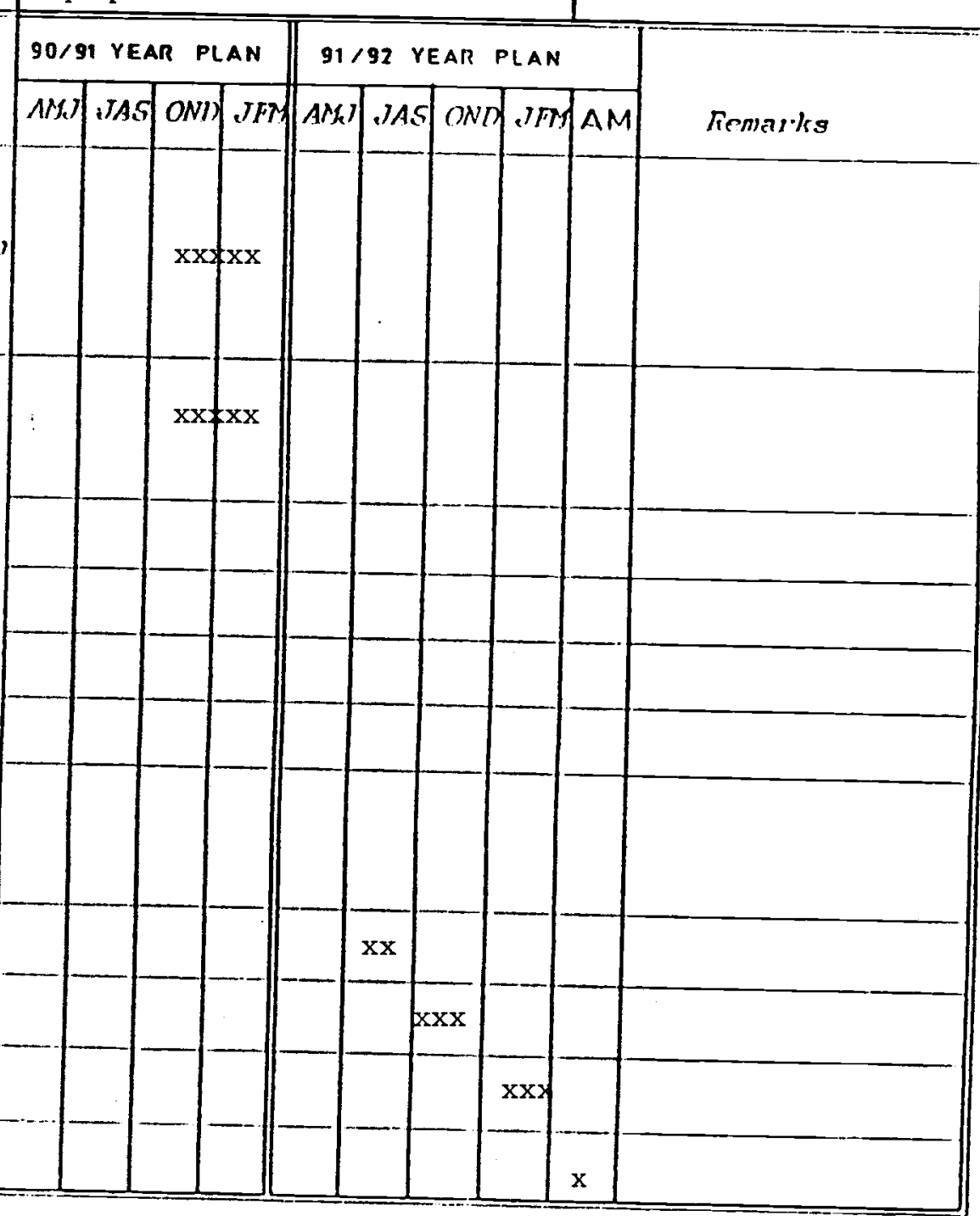




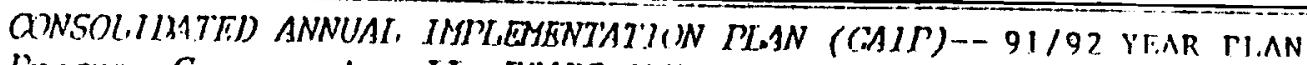

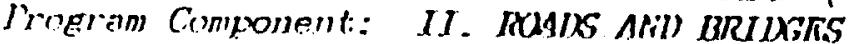

ARS Nimber:

07

ARS Name: Paktia

Irice 1 or 1

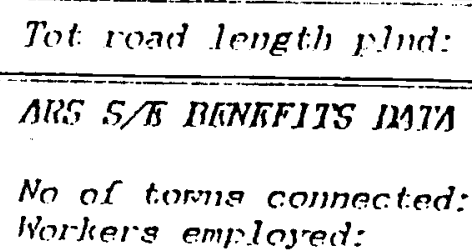

ARG TKCIIACBSISITTC RROMTS

Surrey/design assistance:

Materials:

Equigment:

Tools:

All material and equipment supplied from local bazar

ARS TIMRI.LNR

Activityr

1. Plaming: ldentify gubprojects, couduct reasibility studie.3, prioritize, obtain local approval, complote $1 \hat{R}-1$ and $I R-2$ forms.

2. Survey. design and preconstruction reports for subproject by priority.

Higlues t ranking 1/\&th:

Next ranking 1/4th:

Next ranking $1 / 4 t h$ :

Lowegt ranking 1/4th:

3. Mobilization and construction of subprojects by priorit: $\mathrm{J}^{\mathrm{t}}$.

Highest: ranking 1/4th:

Next: ranking 1/4til:

Next ranking 1/4th:

Lowest ranlsing J/Ath:

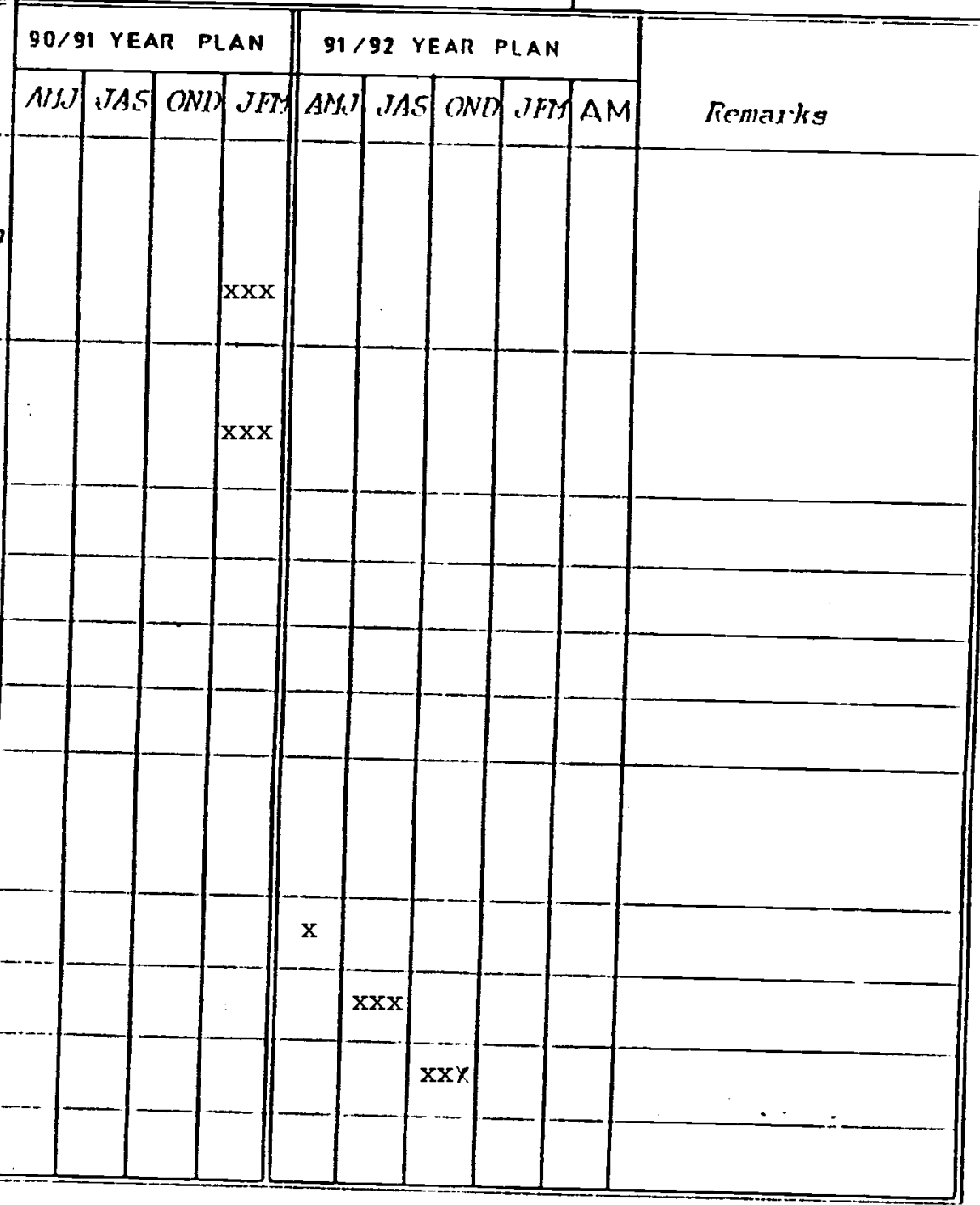


OONSOLIMATED ANNUAL, INTLIOMFNTAIION PLAN (CAIP)-- $91 / 92$ YFAR TI,AN

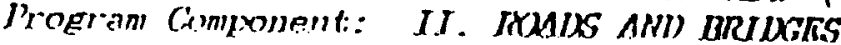

AR.S Numlier:

ARS Name: Konar

PrEe 1 or 1

Tot road length plud: $75 \mathrm{~km}$ Thtal bridgeg plammed:

ARS S/K HINKFITS DATA

No of torng comnectied: 8

Workers enployred: 312,000

ARS TKIIIRAXISITTC RRKMTS

Survey/design asgigtance:

Materials:

Equizment:

7'ool.9:

All tools and mater al supplied from local bazar

ARS TIMTI,INK

Activity

1. Plaming: Ictentify subrojects, conduct feasibility studies, prioritize, obtain local ipproval, complete $1 R-1$ and $I R-2$ forms.

2. Survey, design and preconstruction report.g for subproject by priority.

Higheg: ranking $1 / 4 t h$ :

Next ranking I/4th:

Next ranking 1/4t:J:

Lowegt: rasking 1/4th:

3. Nobilization and construction of subprojects ly prioril.y.

lighest ranking $1 / 4 t h:$

Next: ranlsing 1/4th:

Next ranking j/dth:

lenwest: lanking 1/Ath:

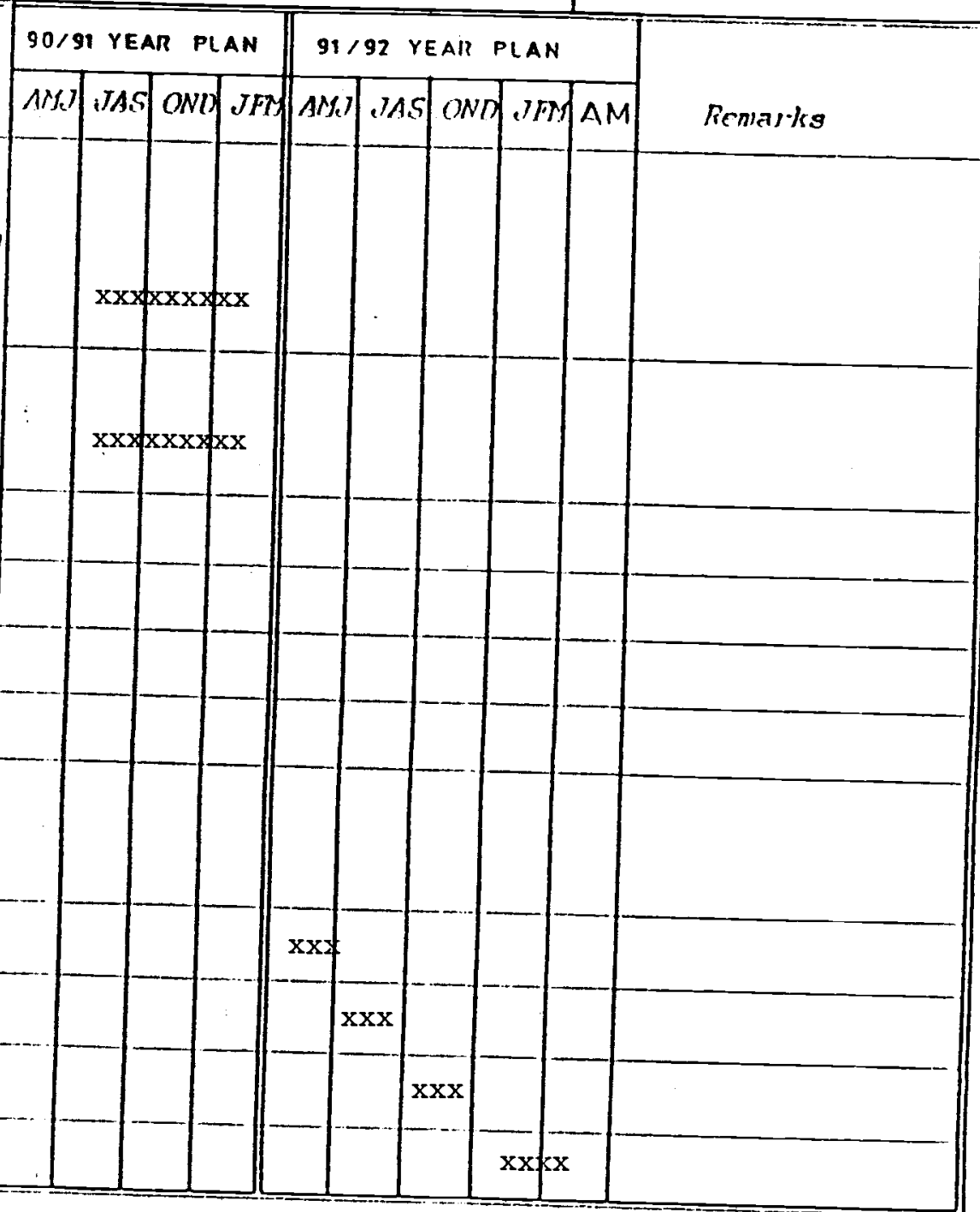


OONSOLIIKJTFI) ANNUAL, IMITIEMENTATION RLAN (CAIP)-- 91/92 YFAR TI,AN

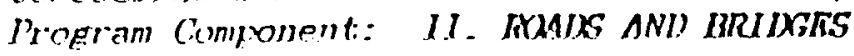

ARS Numler: 12

IRS Name: Takhar

Srige 1 of 1

Tot road length Find: $42 \mathrm{~km}$

ARS $5, \pi$ DKNKFITS MM7'

No of tomis commected:

Morkerg employed: 472

RH lind (Ra):1,570,000

ARS TK(HIRKAISITIC RRAYIS

Surver/design assigtance:

Materials:

Fquipment:

7ools:

Ali tools and equipment supplied from local bazkr

ARS TIMTLINE

Activity

1. Flanning: Identily subprojects, conduct feasibility stulies, prioritize, obtain local approval, complete $1 R-1$ and $I \pi-2$ forns.

2. Surtrey, design and preconstruction reports for subproject by priority.

Highesit ranking 1/4th:

Next ranking 1/4th:

Next ranking 1/4th:

Inregt. ranking 1/4th:

3. Molitization and construction of subprojects by priorily.

Higliegt ranking $1 / q t h$ :

Next: ranking 1/4t:h:

Noxt ranking $\mathrm{g} / \mathrm{dth}$ :

Loweat ranking 1/Ath:

\begin{tabular}{|c|c|}
\hline 90/91 YEAR PLAN & $91 / 92$ YEAR PLAN \\
\hline$A M O T A S$ OND JFT & AMN JAS OND TFM AM \\
\hline
\end{tabular}

Total Carm ramilies: 18,650

Total Jerils: $\quad 27,500$

Nescription
Total employment:

Shilled: $655 \mathrm{mel}$

linglitled: $71,250 \mathrm{met}$

Men needed

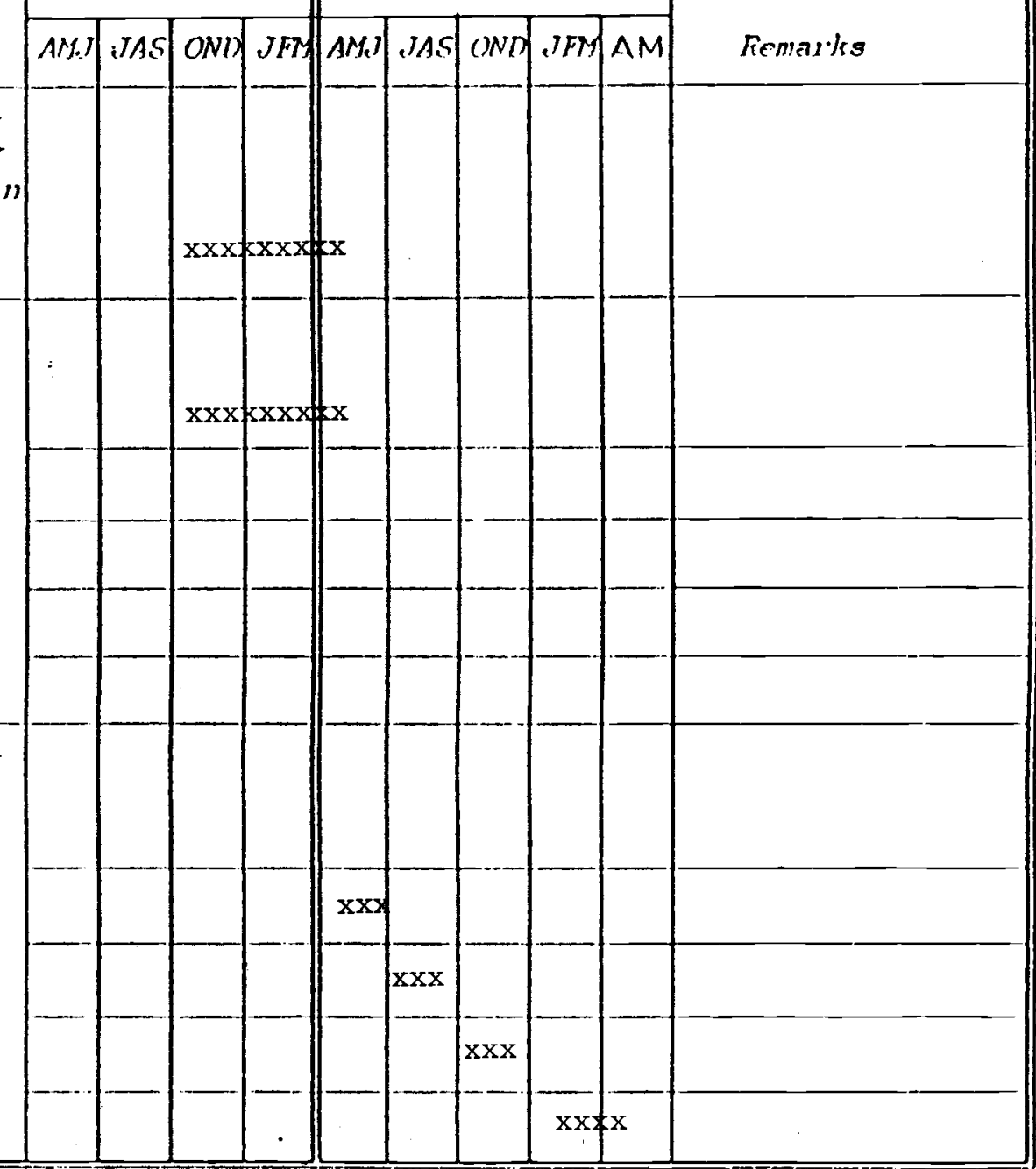


CONSOL.IIBTTED ANNUAL IMPLEMENTATION PLAN (CAIP)-- $91 / 92$ YF.AR TI.AN

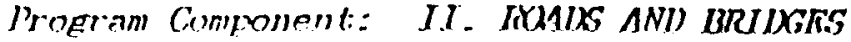

ARS Nimber: 13 ARS Name: Baghlan

NO.

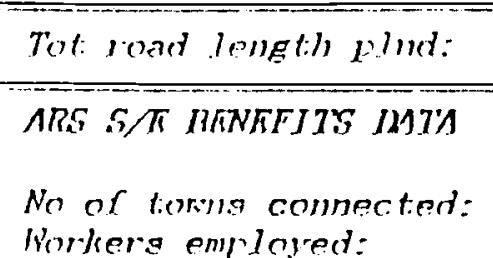

Norkers emlayed:

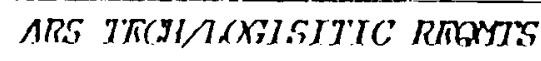

Ginrey/design assiglance:

Materials:

Equizment:

7ools:

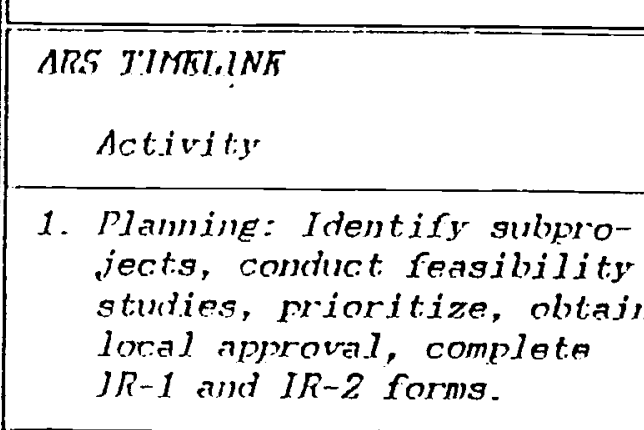

2. Survey, design and preconstruction reports for subproiect by priority.

Highest ranking 1/4th:

Next ranking 1/4th:

Next ranking 1/gt:h:

Lowent: ranking 1/4th:

3. Mobilization and construction of subprojects by priorit:yr.

Highest ranking 1/4th:

Next ranking 1/4th:

Nert ranking $1 / 4 t h$ :

Lwest: ranking 1/4th:

\begin{tabular}{|c|c|c|c|c|c|c|c|c|c|}
\hline \multicolumn{4}{|c|}{$90 / 91$ YEAR PLAN } & \multicolumn{5}{|c|}{$91 / 92$ YEAR PLAN } & \multirow[b]{2}{*}{ Femarks } \\
\hline$A M_{2}$ & JAS & $O N I$ & $J F M$ & $A M_{2}$ &.$T A S$ & OND &.$J F M$ & $A M$ & \\
\hline
\end{tabular}


OONSOL.IHITFD ANNUAL IMILEMENTATIION PLAN (CAIP)-- 91/92 YFAR TI,AN

Program Component: II. INASIXS ANI) RRIIXGKS

ARS Nimber: 14

ARS Name: Kunduz

IriEe 1 or 1

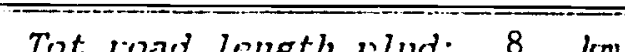

Total brideeg nlanes: 8

ARS $5 / \bar{T}$ NFNIFITS IMTM

No of temsma comnected:

Porkers emiloyed:

ARS TKCINCWISTITC RMOMTS

Surver/iesign assistance:

Materials:

Equigment:

Tools:

Ali materials, qquipment and tools supplied froh local bazar

ARS TIMELINK

Activitir

1. Plaming: Icientify subprojects, conduct feasibility stulies, prioritize, obtain local approval, complete $i R-1$ and $I R-2$ forms.

2. Survey. degign aud preconstiruction reports for subproiect by priority.

Highegt ranking $1 / 48 h_{3}$ :

Next ranking 1/4th:

Next ranking $1 / 4 t$ t :

Lorear: ranking 1/4th:

3. Nobilization and construction of subprojects by priority.

Highest ranking 1/4th:

Next: ranking 1/4th:

Next ranking $1 / d t$ s:

lerveat ranking 1/4th:

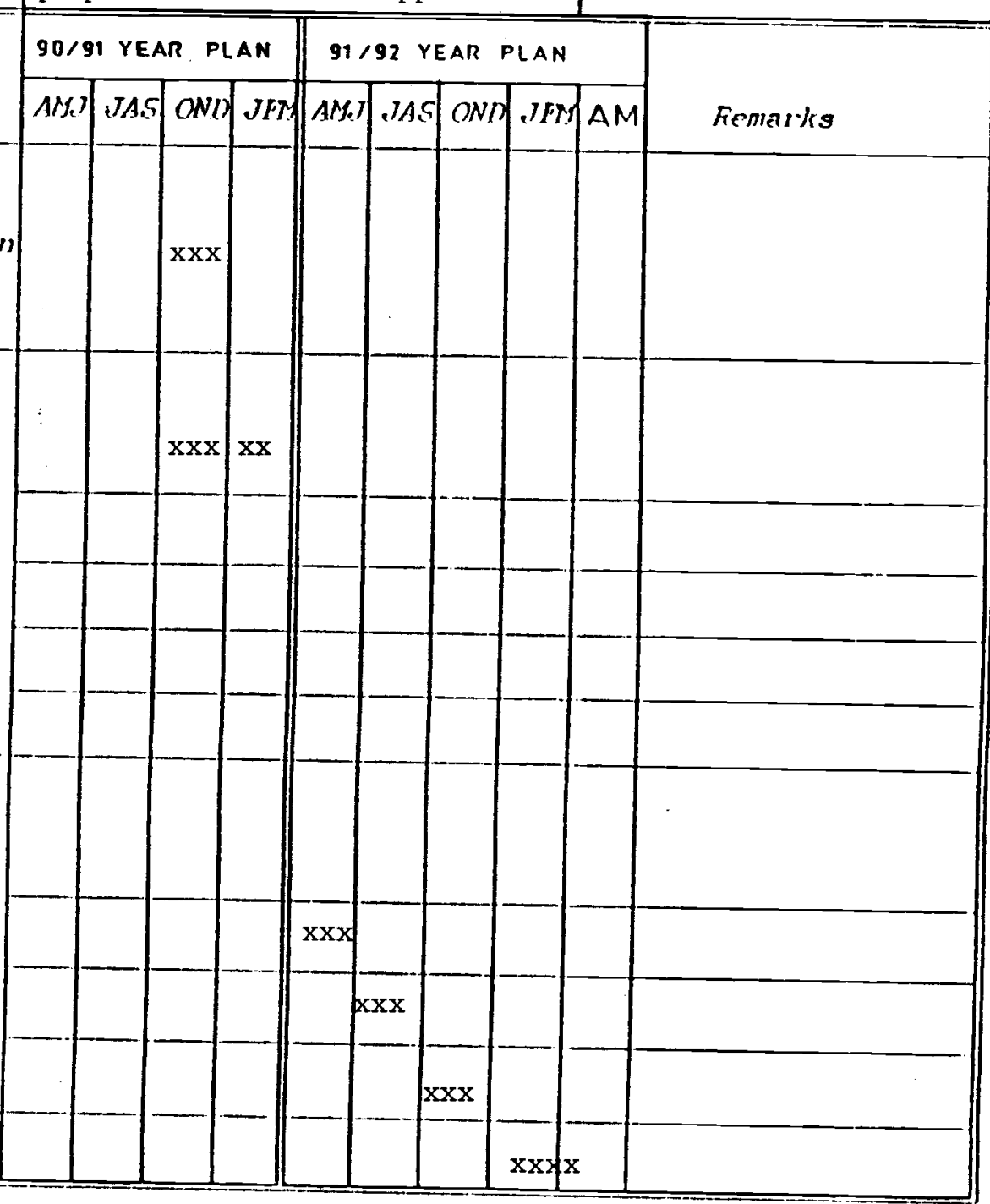


ARS Name: Herat

Prige 1 or 1

\begin{tabular}{|c|c|c|c|}
\hline Tot: eoad longth plud: $28 \mathrm{Jmm}$ & $\begin{array}{c}\text { Total brjdges plamed: } 3 \\
\text { culverts: } 22\end{array}$ & \multicolumn{2}{|c|}{ RB lund (R.9):2,961,350 } \\
\hline ARS $S / K$ LWNEFITS DMTA & Within road influence areas: & 7otal employment & \\
\hline 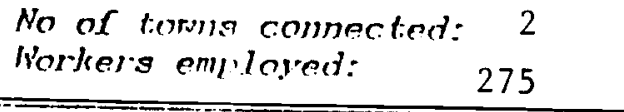 & $\begin{array}{l}\text { Total farm ramilies: } 20,000 \\
\text { Total Jeribs: } 100,000\end{array}$ & $\begin{array}{lr}\text { Skilled: } & 2,550 \\
\text { Unakjlled: } 12,290\end{array}$ & $\begin{array}{l}m d \\
m d d\end{array}$ \\
\hline ARS TKGIЛККВISITIC RRAMIS & Inscription & Menl needed & \\
\hline
\end{tabular}

Survey/design assistance:

Materials:

Equizment: :

Tools:

All equipment, to 1 s and material will be suppliep from local bazar

ARS TIMRLINK

Activity

1. Plannine: Identify subprojects, conduct feagibility studies, prioritize, obtain local ipprotal, complete IR-1 aud IR-2 forms.

2. Survey, design and preconstruction reports for subproiect by priority.

Highes t: ranking 1/4th:

Nert: ranking 1/4th:

Next ranking I/gth:

lowes: ranking $1 / 4 t h$ :

3. Mobilization and congtruction of sulprajects by priority.

Highest: ranking 1/4t, :

Next: ranking 1/4th:

Next reanking J/4th:

larreat ramlsing $1 / 4$ th:

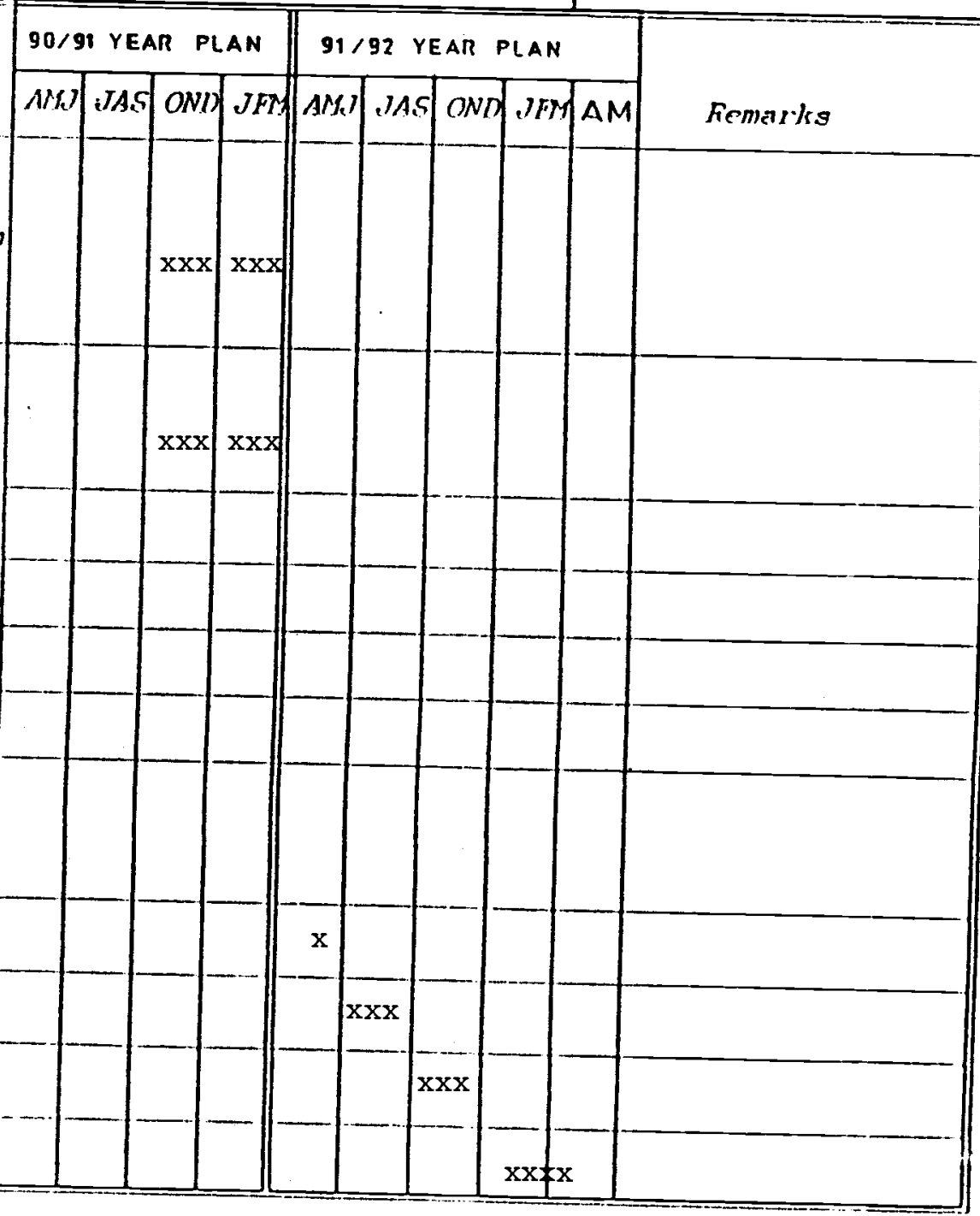


CONSOLIIBATHI) ANNUAL, INILEMENTATILN PLAN (CAIP)-- $91 / 92$ TIAR TI.AN

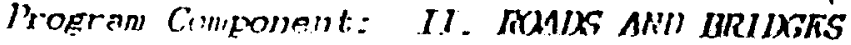

ARS Number:

ifRS Name: Farah

lige 1 of 1

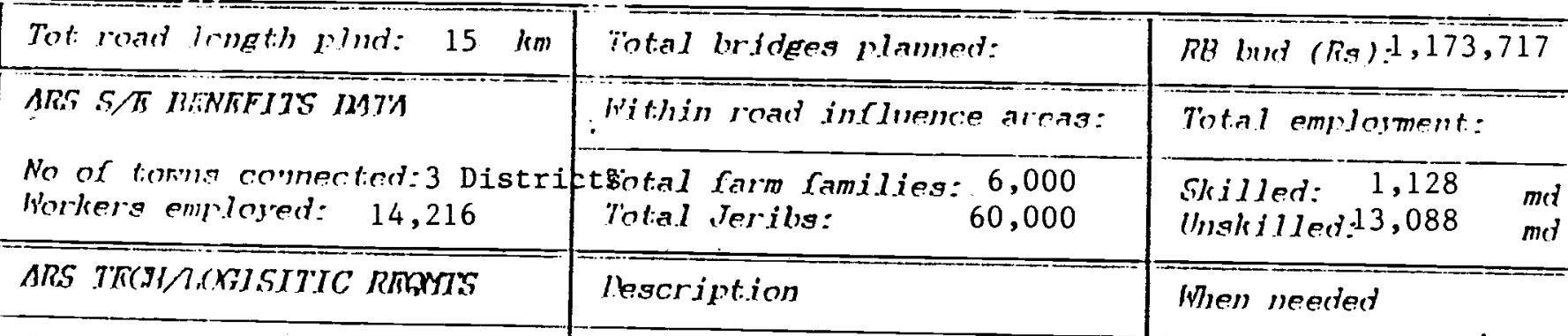

Survey/design asgigtance:

Materials:

Bquipment:

Tools:

All materials, equipment and tools supplied from loc $\$ 1$ bazar

\begin{tabular}{|c|c|}
\hline & $\begin{array}{l}\text { RS TUMRT.INA } \\
\text { Activily }\end{array}$ \\
\hline & $\begin{array}{l}\text { Planning: Identify subpro- } \\
\text { jects, conduct reasibility } \\
\text { studies, prioritize, obtaj } \\
\text { local approval, complete } \\
\text { lR-l and IR-2 forms. }\end{array}$ \\
\hline
\end{tabular}

2. Survey, degign and preconstruction reportis for sublurouect by priority.

Highegt ranking 1/4t:h:

Next: ranking $1 / 4$ th:

Next ranking $1 / \Delta(:)$ :

lowest ranking 1/4th:

3. Mohilimention and construction of subprojects by priority.

Highest: ranking 1/4th:

Next: rinlsing 1/4th:

Next rasking 1/\&th:

Lowest: ranking 1/4tb:

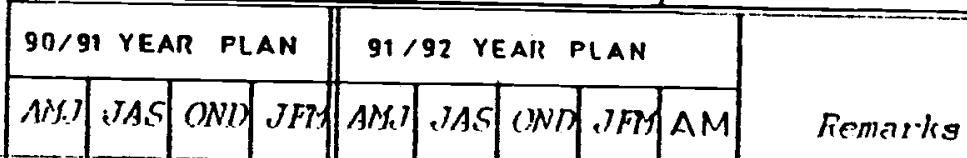




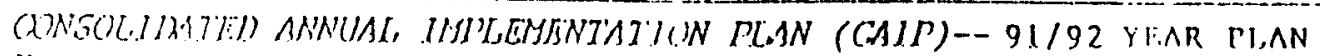

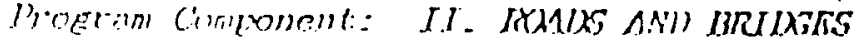

A 1.5 Nimler: :

24

iR.S Name: Kandahar

SiEC _ 1 orl

Tot: rond lenglh plat: 66 lom AŔ

himpera emiloyed:

$+\cdots$

Thtal budgeg plammed: Within roas influence areas:

FH lnet (R.9): 586,998

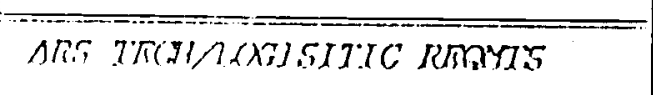

chetal farm Camilies: 511,968 Tolial Jerilus: 48,600

Itogcription

Total emploiment:

Skijled: $2,320 \mathrm{met}$

Unsirilled:67,230 mi

Sumer/dosign agaistance:

Materials:

Equipment:

7oolo:

We are supplying all faterials, equipment and tools from local bazar

ARS TIMELINT

Activily

1. Plaming: Identify subproject.g, couduct feasibility stucites, fricritize, obtajn local aproval, complete lh-l and IR-2 forms.

2. Surteyr. design and preconstrict:ion reports for subproject by priority.

Highesi: ranking $1 /$ Qt:h:

Noxt: rasking 1/\$th:

Next: trnking $1 / 4$ th:

Lorwes: teanleing $1 / 4$ th:

3. Molilitiation and construction of suhprojects ly riorit.y.

lighest: rawhing $1 / 4$ th:

Next ramlisig 1/4kh:

Next ;anking $\mathrm{g} / \Delta \mathrm{t}$ ):

forroot ranking $l / A(b)$ :

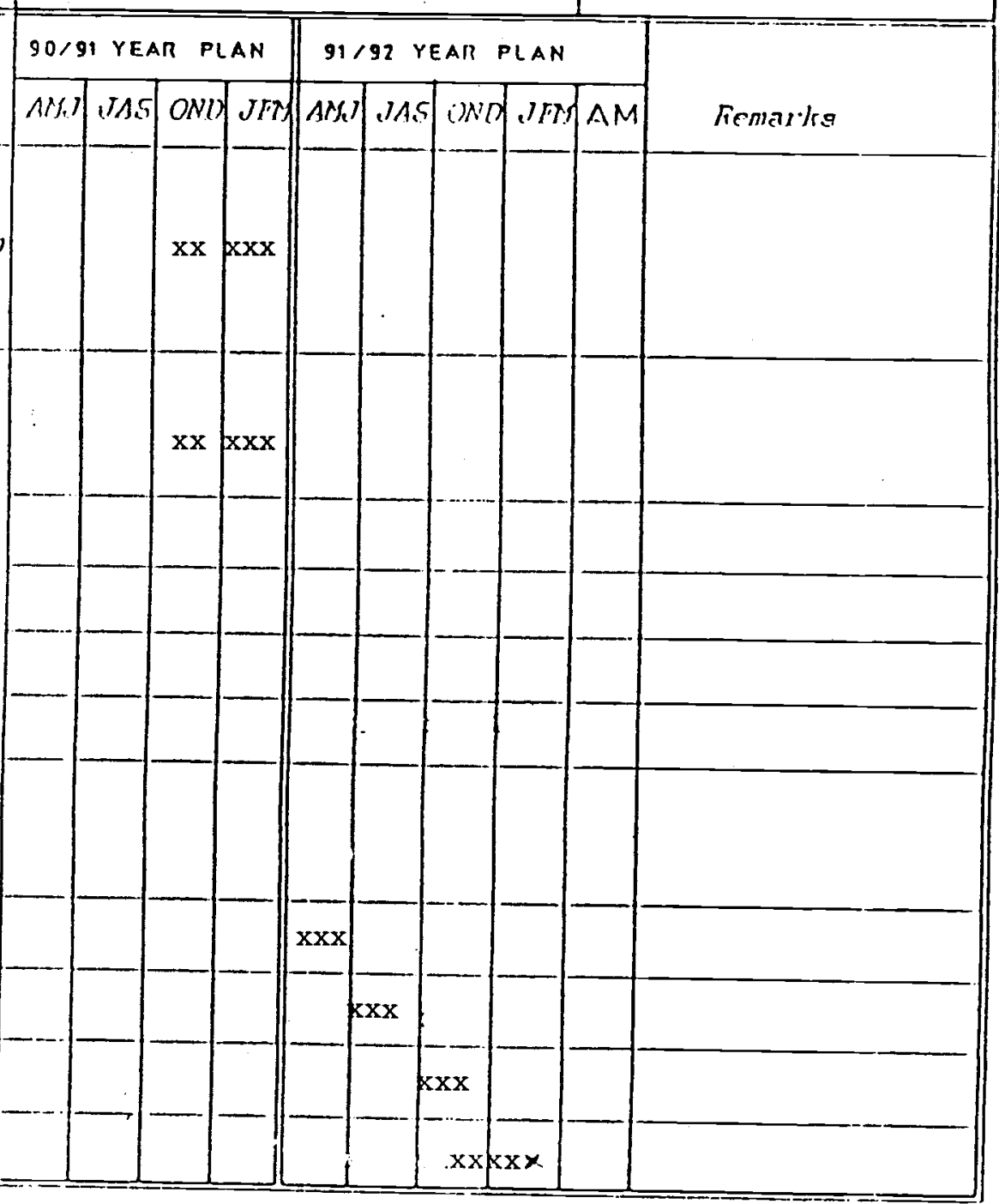


Program Component: : IT. IKAIJS ANI BRIDKTS

ARS Nimler: $\quad 28$

ARS Name: Bamyan

Irige 1- of 1

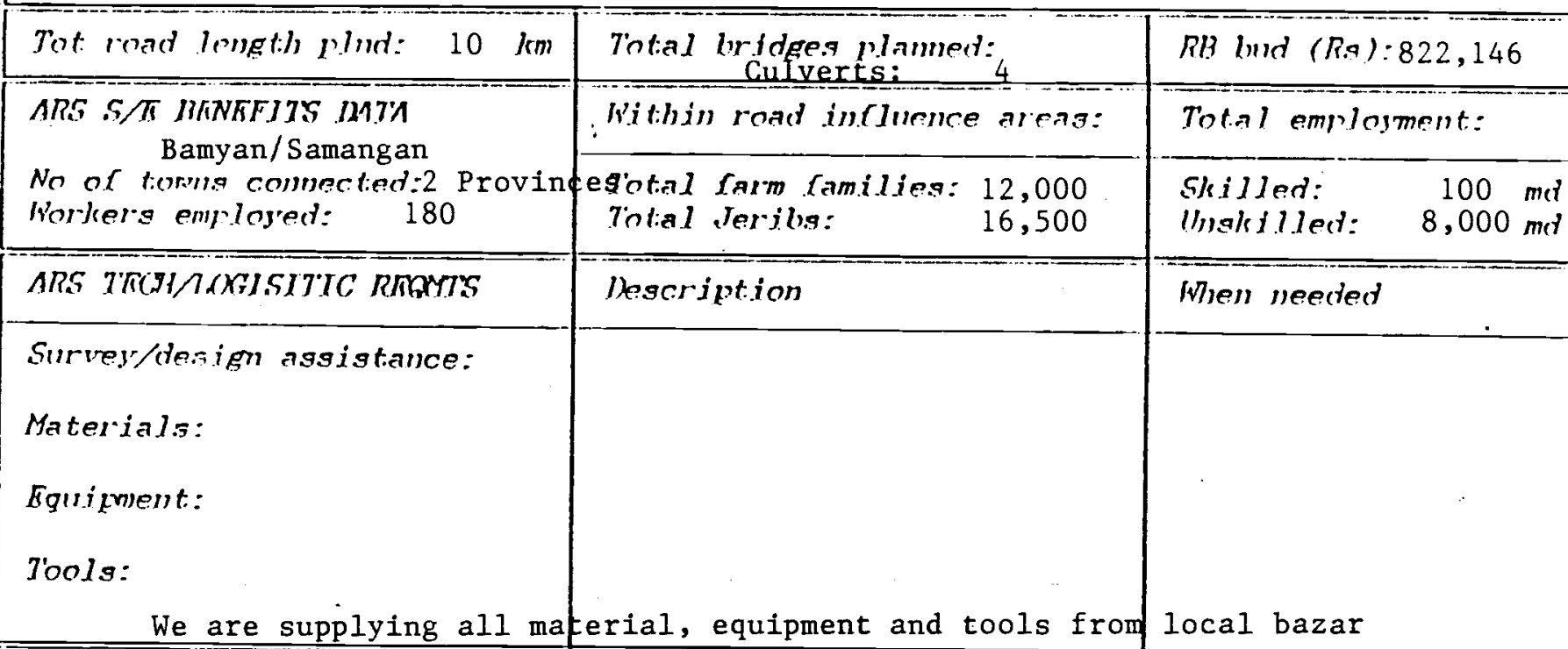

ARS TIMMLINK

Activil:y

1. Plaming: Identify subprojects, conduct reasibility studieg, prioritize, obtajn local approval, complete IR-1 and $I R-2$ forms.

2. Survey. design and preconstruction reportig for subproject by priority.

Highest ranking 1/4th:

Next ranking $1 / \phi$ th:

Next ranking $1 / 4$ th:

Lorvest: ranking $1 / 4$ th:

3. Mobilization and construction of subprojects by priority.

Higheat: ranking 1/4th:

Next rrolking 1/4th:

Next ranking 1/dth:

larsent: ranking I/Ath:

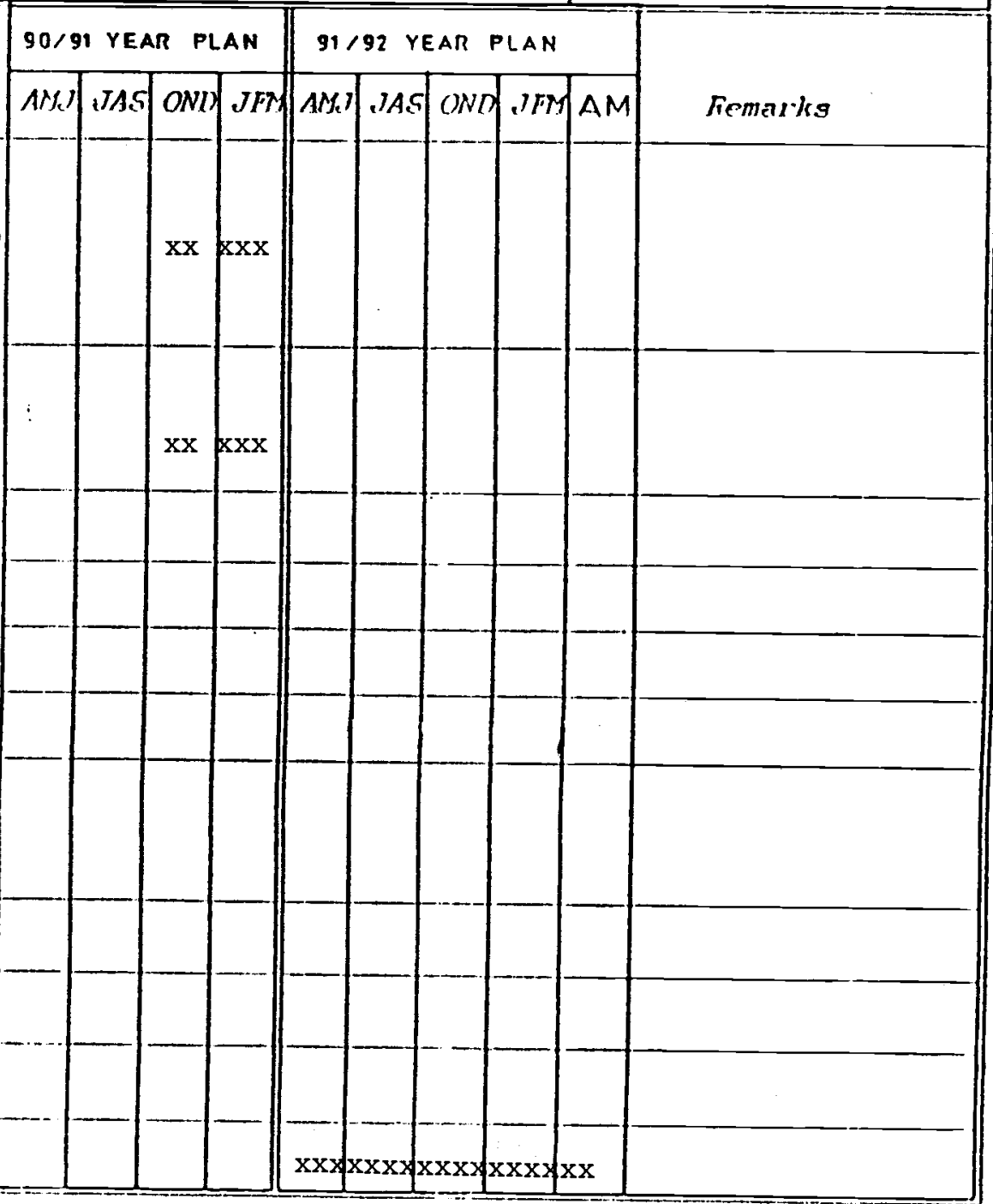


OONSOLINMTTED ANNUAL INITLEMENTATION PLAN (CAIP)-- 91/92 YFAR TI.AN

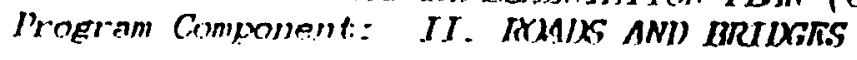

Form $R B-2$

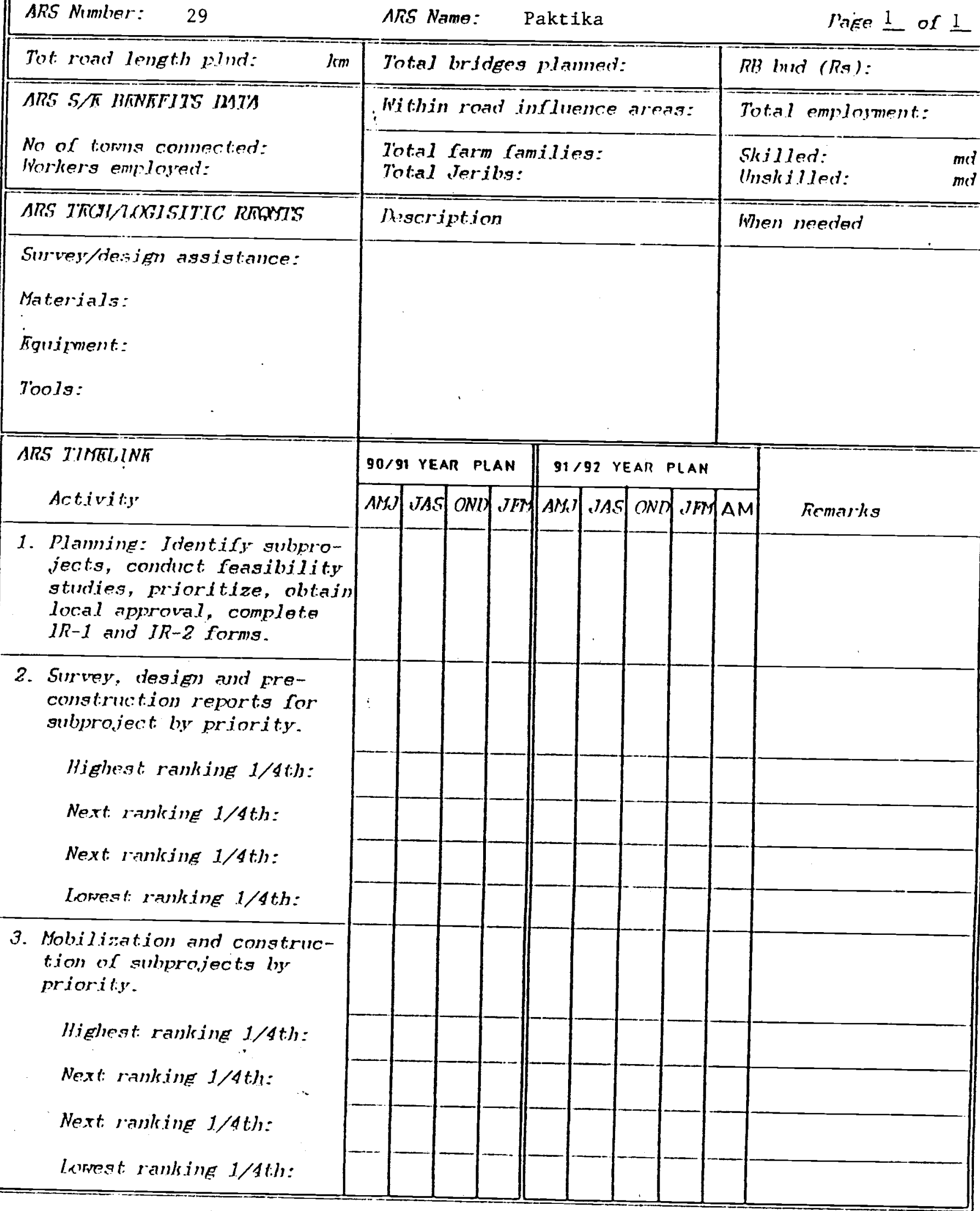




\author{
VITA/LLBI - CONTRACT
}

SCOPE OF WORK:

In carrying out its work in rural rehabilitation, the ARR staff ridy occasionaliy encounter a need for spacialized or complex enginecring services which exceed in-house capabilities. For examplt, design recommendaiions may, a some future time, be needed for focilities such as long-span bridges, large diversion structures, dams or powex generation facilities. Also, general advisory services may be needed related to the ongoing rural works progrars, in areas such as system analysis and the development of design standards for relatively simple facilities.

In order to meet the this need, VITA has entered into this "work order-type" contract with Louis Berger International, Inc. (LBII). LBII is willing to provide staff and facilities on short notice to carry out the following activities in support of repair and rehabilitation of physical infrastructure:

- temporary assignment of personnel to the ARR office in Peshwar, Pakistan

- reconnaissance trips inside Afghanistan for assessments, evaluations and preparation of technical recommendations,

- preparation of design sketches, outline specifications and cost estimates for the guidance of ARR staff, who would complete the design work,

- supervision of desigr work to be undertaken by the ARR staff, temporary local staff or local engineering firms that would bc subcontracted by VITA,

- execution of design work, using the firm's own in-house staff at its home or brancis offices.

Design work referred to above may include the preparation of working drawings specifications, cost estimates and other contract documents to be used in contracting with local or international construction firms.

For each assignment under this subcontract the contractor in consultation with the subcontractor will prepare a delivery order specifying the work to be accomplished, the timing of the assignment, the personnel to be assigned and a budget. The Delivery order will be signed by both parties and forwarded to USAID for their information. 


\begin{tabular}{|c|c|c|c|c|c|c|}
\hline $\begin{array}{l}0 \\
\frac{x}{\alpha} \\
\frac{\alpha}{\alpha} \\
\frac{\pi}{2} \\
\underline{\alpha}\end{array}$ & 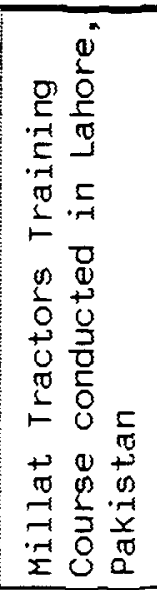 & $\begin{array}{l}0 \\
2 \\
0 \\
0 \\
0 \\
0 \\
0 \\
0 \\
0 \\
5 \\
0 \\
0 \\
0\end{array}$ & 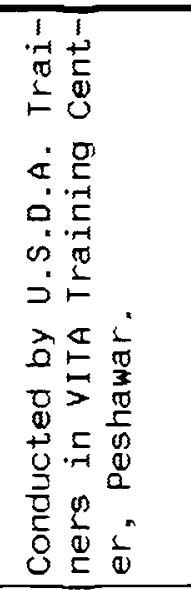 & 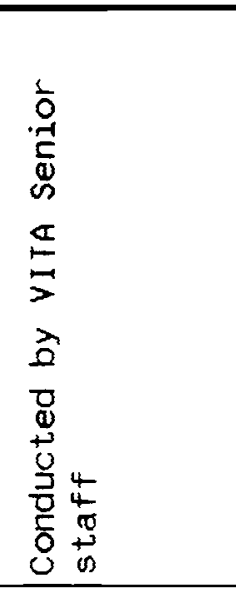 & $\begin{array}{l}1 \\
0 \\
0 \\
0 \\
0 \\
0 \\
5 \\
5 \\
0 \\
0 \\
0 \\
0 \\
0 \\
0 \\
0 \\
0 \\
0 \\
0 \\
0 \\
0 \\
0 \\
0\end{array}$ & 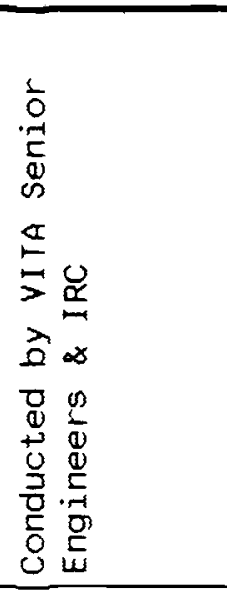 \\
\hline 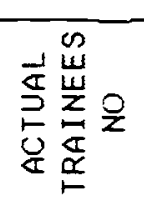 & $\infty$ & r & $\vec{N}$ & $\approx$ & $\stackrel{0}{\circ}$ & $\stackrel{\sim}{N}$ \\
\hline 운 & $\stackrel{\sim}{N}$ & $\stackrel{0}{-}$ & \pm & $2+$ & $\stackrel{0+}{-4}$ & पे \\
\hline 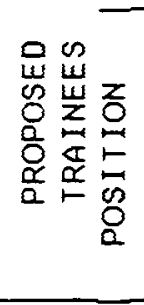 & 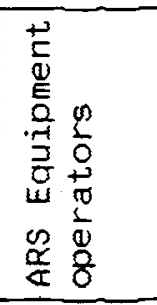 & 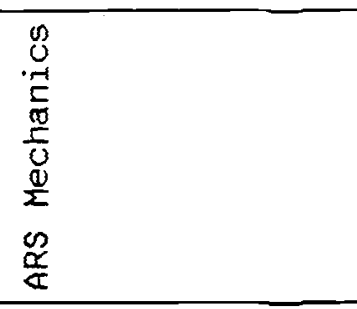 & 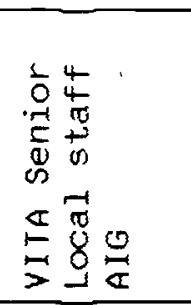 & 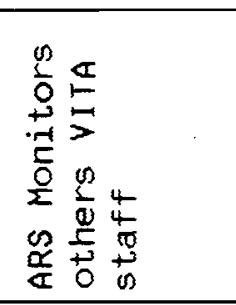 & 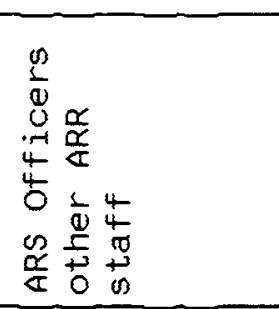 & 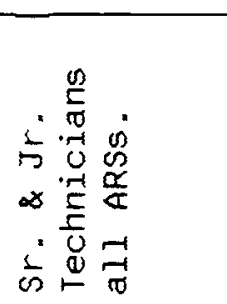 \\
\hline 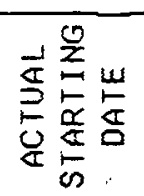 & \begin{tabular}{l}
$\stackrel{0}{2}$ \\
0 \\
0 \\
0 \\
\hdashline \\
-1
\end{tabular} & $\begin{array}{l}\stackrel{2}{2} \\
\stackrel{2}{2} \\
\stackrel{-1}{-1}\end{array}$ & $\underset{\substack{\infty \\
\sim}}{\stackrel{\alpha}{\alpha}}$ & $\underset{5}{5} \stackrel{\circ}{\circ}$ & $\begin{array}{l}\overrightarrow{2} \\
\frac{a}{2} \\
\Rightarrow\end{array}$ & 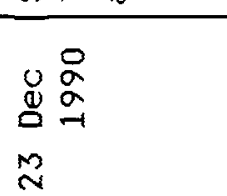 \\
\hline 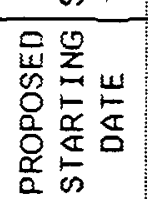 & $\begin{array}{l}1 \\
2 \\
0 \\
0 \\
0 \\
N\end{array}$ & $\begin{array}{l}2 \\
2 \\
0 \\
0 \\
N\end{array}$ & $\begin{array}{l}8 \\
2 \\
5 \\
5 \\
m \\
m\end{array}$ & $\begin{array}{l}8 \\
2 \\
5 \\
5 \\
0\end{array}$ & 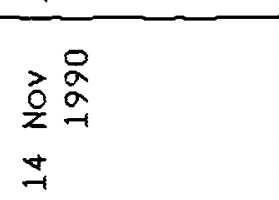 & 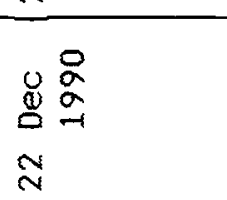 \\
\hline 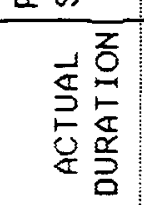 & $\begin{array}{l}0 \\
\frac{0}{0} \\
\frac{0}{3} \\
4\end{array}$ & $\begin{array}{l}0 \\
\frac{9}{0} \\
\frac{1}{3} \\
3 \\
+\end{array}$ & $\begin{array}{l}n \\
\frac{n}{w} \\
\tilde{W} \\
N \\
N\end{array}$ & $\begin{array}{l}n \\
\frac{w}{w} \\
\frac{w}{3} \\
N\end{array}$ & $\begin{array}{l}n \\
\frac{n}{0} \\
\frac{0}{3} \\
\frac{0}{3} \\
N\end{array}$ & $\begin{array}{l}0 \\
\frac{0}{0} \\
0 \\
\frac{0}{3} \\
0 \\
-1\end{array}$ \\
\hline 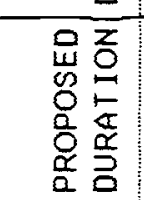 & $\begin{array}{l}\frac{0}{0} \\
\frac{w}{3} \\
+\end{array}$ & $\begin{array}{l}0 \\
\frac{2}{0} \\
\mathbb{W} \\
+ \\
+\end{array}$ & $\begin{array}{l}\frac{n}{2} \\
\frac{0}{0} \\
3 \\
+\end{array}$ & $\begin{array}{l}\stackrel{0}{2} \\
\stackrel{W}{\mathbb{W}} \\
\mathbf{3} \\
N\end{array}$ & $\begin{array}{l}\frac{n}{0} \\
\frac{0}{W} \\
\frac{w}{3} \\
N\end{array}$ & $\begin{array}{l}\frac{0}{0} \\
\frac{v}{0} \\
\frac{0}{3} \\
0 \\
0\end{array}$ \\
\hline 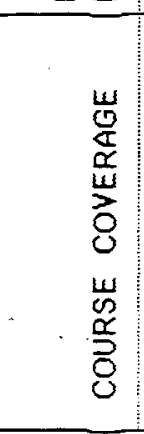 & 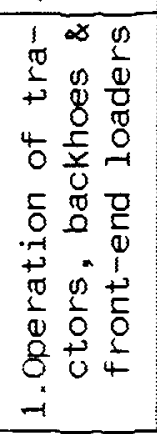 & 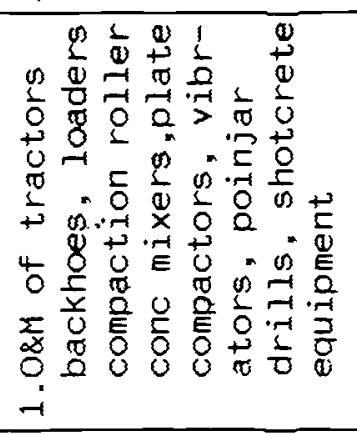 & 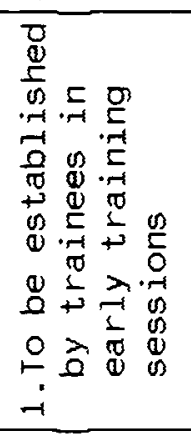 & 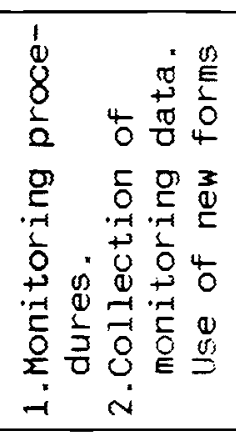 & 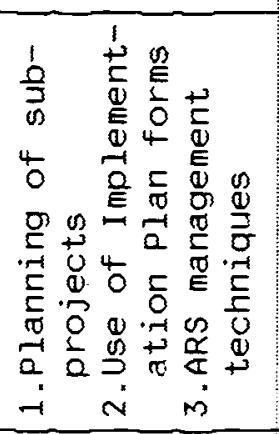 & 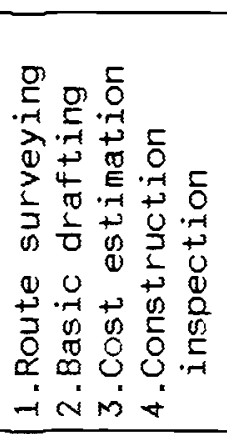 \\
\hline $\begin{array}{l}\frac{w}{\Sigma} \\
\frac{\sigma}{z} \\
w \\
\omega \\
\frac{\alpha}{3} \\
\delta\end{array}$ & 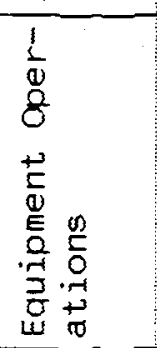 & 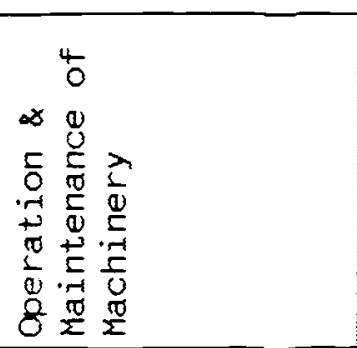 & 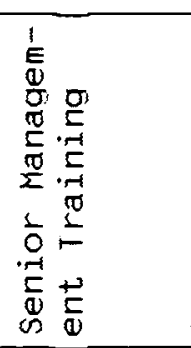 & 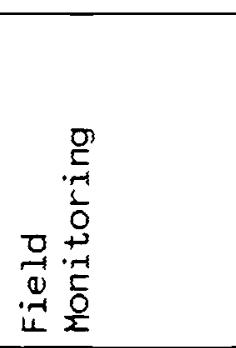 & 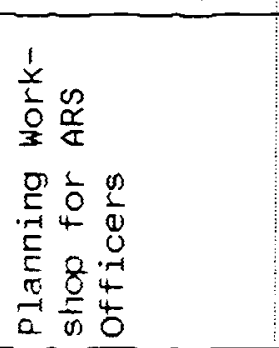 & 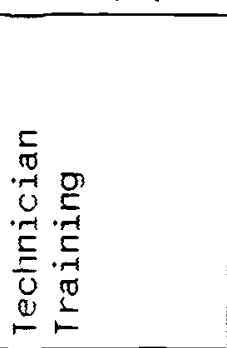 \\
\hline$\frac{0}{2}$ & $\rightarrow$ & $N$ & $\mathrm{~m}$ & $\forall$ & an & $\infty$ \\
\hline
\end{tabular}




\begin{tabular}{|c|c|c|c|c|c|c|}
\hline 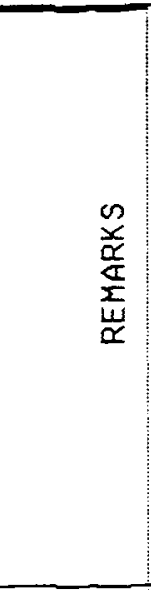 & 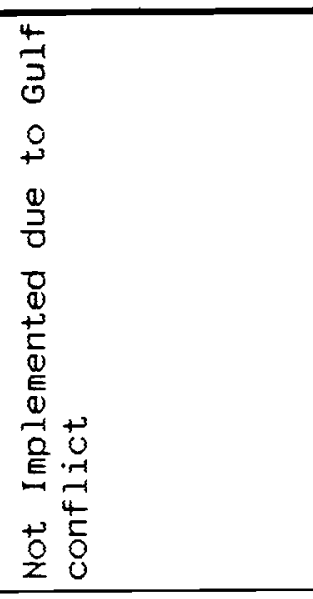 & 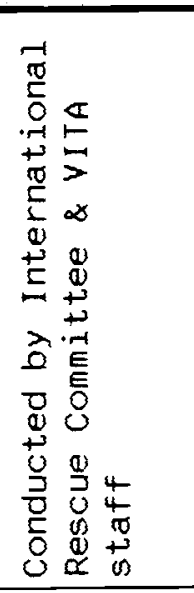 & 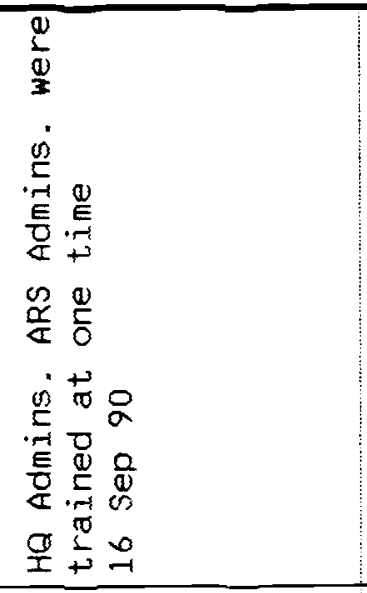 & 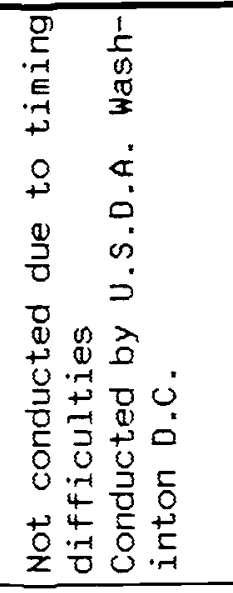 & 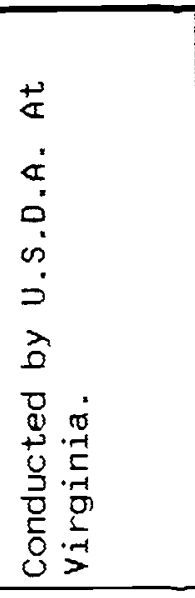 & 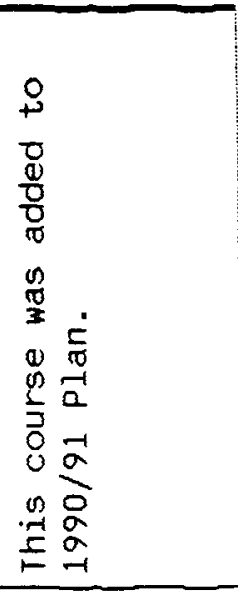 \\
\hline 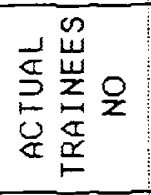 & & $\vec{\lambda}$ & 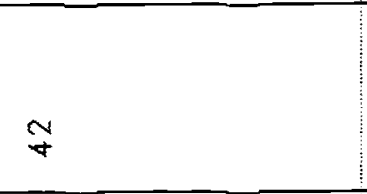 & $0-1$ & $\mathrm{~m}$ & $m$ \\
\hline$\stackrel{p}{z}$ & & 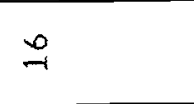 & $\stackrel{\sim}{ }$ & $\sim-1$ & $m$ & $i$ \\
\hline 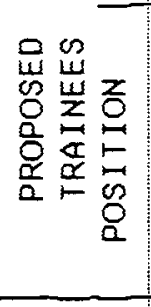 & 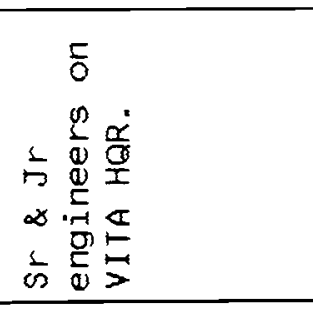 & 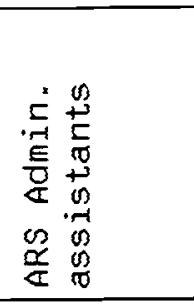 & 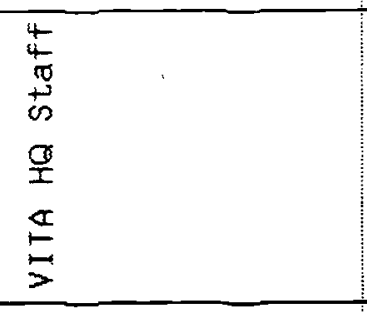 & 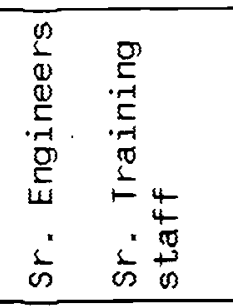 & 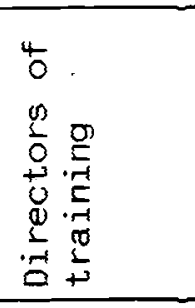 & $\begin{array}{l}\frac{0}{0} \\
\frac{0}{2}\end{array}$ \\
\hline 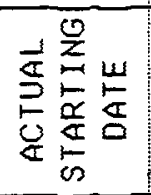 & & 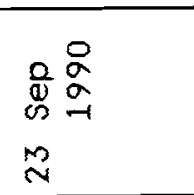 & 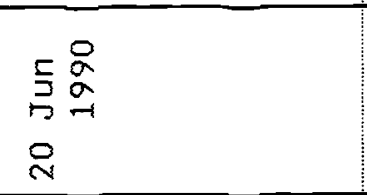 & $\begin{array}{l}9 \\
\stackrel{9}{9} \\
0 \\
\end{array}$ & 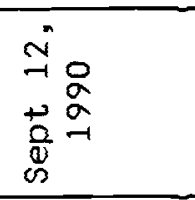 & $\frac{2}{2}$ \\
\hline 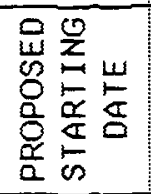 & 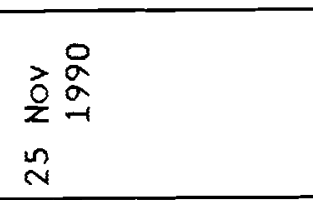 & 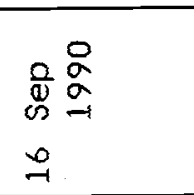 & 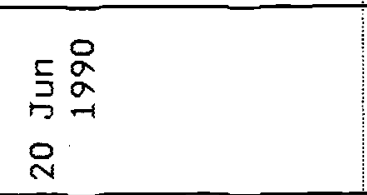 & $\begin{array}{l}1 \quad \frac{9}{E} \\
1 \\
0\end{array}$ & 今ं & 1 \\
\hline 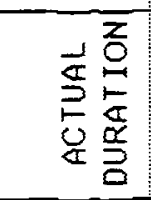 & & 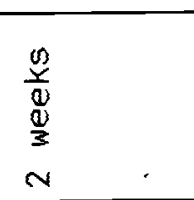 & 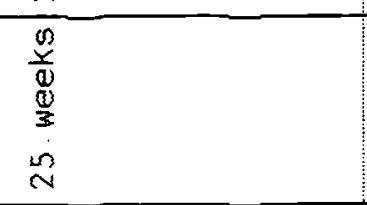 & 1 & 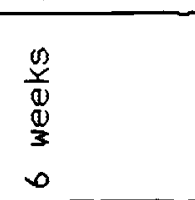 & 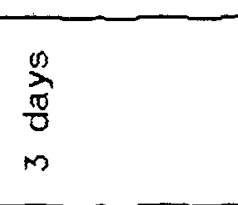 \\
\hline 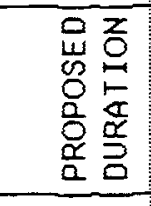 & $\begin{array}{r}\frac{\infty}{0} \\
\frac{1}{0} \\
3 \\
+ \\
\end{array}$ & 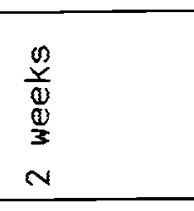 & 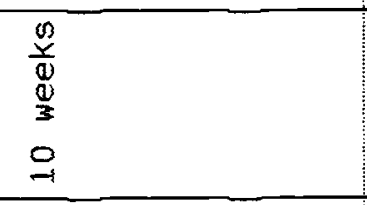 & 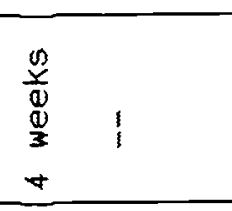 & $\begin{array}{l}\frac{n}{0} \\
\mathbb{0} \\
\mathbb{W} \\
\mathbf{3} \\
0 \\
\end{array}$ & 1 \\
\hline 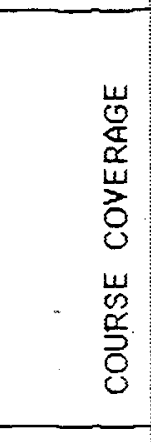 & 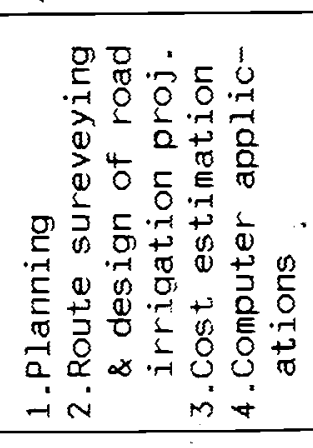 & 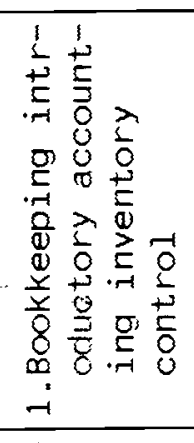 & 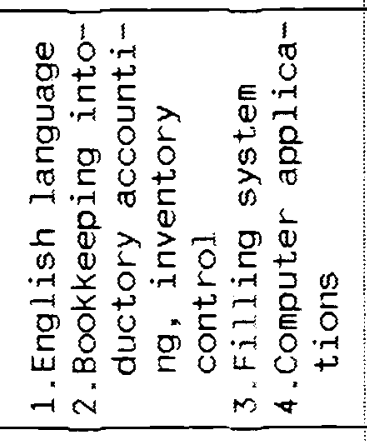 & 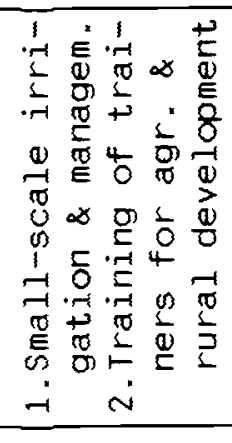 & 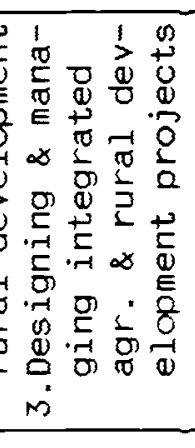 & 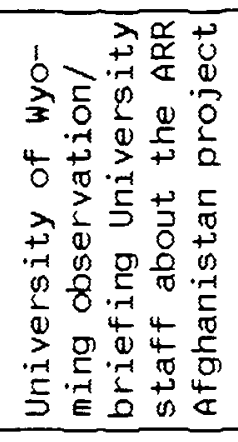 \\
\hline 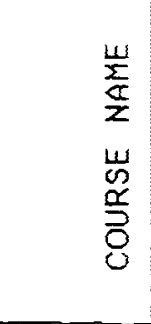 & 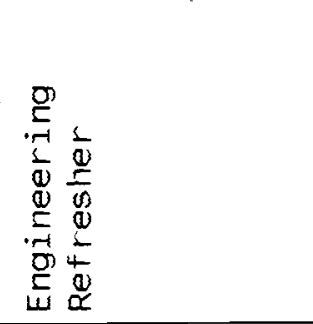 & 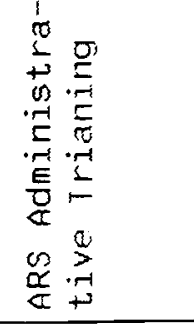 & 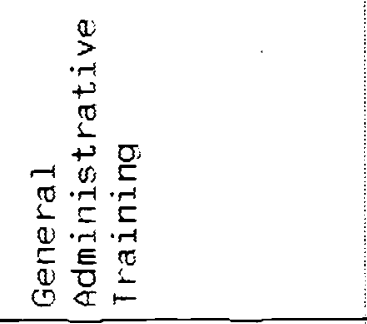 & 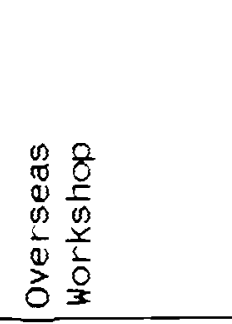 & & 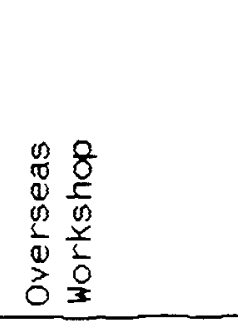 \\
\hline$\frac{0}{2}$ & r & $\infty$ & $a$ & 9 & & $\exists$ \\
\hline
\end{tabular}




\section{VITA-CID-UW TRAINING PROGRAM FOR AFGHAN ENGINEERS AND TECHINICIANS}

\section{INTRODUCTION}

The transfer of technology by upgrading human resources is vital to the success of any development process. This is particularly true for those involved in technical areas because of the need to refresh skills and to upgrade skills and apply improved technology to the solution of problems.

The People of Afghanistan are faced with the problem of rebuilding their Country following an unfortunate war which continues today on a limited scale. Most roads, bridges and irrigation systems have been destroyed. Through the efforts of Afghan engineers and technicians, most of whom are refugees living in Pakistan and the support of donor agencies such as AID and the assistance of organizations such as VITA, much has been accomplished to improve the quality of life of the people in Afghanistan. However, the number of requests for assistance exceeds the capability of VITA staff, and some locations are very difficult to reach, particularly with needed construction materials.

Most technically trained persons were forced to leave Afghanistan during the war and now live in Pakistan as refugees. While these persons received excellent training at Kabul University and similar institutions, they have not received training for more than twelve years except for training provided recently through VITA. Many have not participated in engineering or construction projects for many years. To apply the appropriate technology and engineering skills to problems faced by Afghanistan people, refresher and advanced training is needed.

Typically, the VITA organization provides and engineering design for a specific problem. The design is done in Pakistan with reconnaissance information obtained by technicians in the field. After the design has been completed and approved, a technician travels back into Afghanistan with the essential construction materials to actually construct the project. The technician serves as the construction supervisor and coordinates the efforts of the local people who build the project. The VITA staff must be highly commended for their concern and perseverance in assisting the people of Afghanistan in rebuilding their country. Clearly, much has been accomplished with very limited resources.

VITA recognized the need for training and entered into a cooperative agreement with the Consortium for International Development. The University of Wyoming was appointed Lead University to develop a training program to refresh and

APPENDIX ' $\mathrm{H}$ '

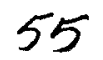

$\mathrm{H}-1 / 10$ 
upgrade VITA engineers and technicians during the winter of 1990-91. However, due to the Middle East War and resulting political unrest, the program was postponed until winter of 1991-92. This schedule is due to the fact that the VITA engineers and technician are active with projects in Afghanistan during the remaining months and are in Pakistan only during the winter. During March 1991, members of the CID-UW team visited VITA in Washington, DC. Team members discussed training needs with VITA and AID personnel, and a verbal report was given at the end of the meetings. This report covers recommendations of the team.

\section{TRAINING TEAM OBJECTIVES}

The overall objective of this training program is to support VITA in their efforts to serve the people of Afghanistan by enhancing the capability of VITA engineering and technical staff. Specific program objectives are:

1. To access the background of participants and refresh and update previously learned English language and technical skills.

2. To refresh engineering fundamentals for engineers and technicians serving on the VITA staff.

3. To enhance the expertise of VITA engineers and technicians in the specialty areas of route surveying, roadways, water supply, and irrigation.

\section{TRAINING PROGRAM}

The training program will include several interrelated elements including skills, assessment, technical English, engineering fundamentals and advanced level courses. Very importantly, the scope and level of the training will depend upon the basic skills assessment which will be conducted during the first few days of the program. Meetings with VITA personnel and others knowledgeable of individuals involved and skills needed have been very worthwhile. The first few days of the actual training program will allow instructors to make necessary adjustments based on first hand interaction with participants.

The overall scope and flow of the training program is illustrated in Figure 1. Referring to the figure, note that all participants will receive the first two program elements which include skill assessment and technical English. Following these, technicians and engineers will be separated for a review of the fundamentals of engineering. The final elements of the training program will provide advanced training. Again, the subjects will be similar, but the emphasis will be different for the engineers

and technicians. Engineering training will focus on project design and planning, whereas technician training will focus on project implementation, construction and management. Participants will select one of the advanced courses based on their individual interests and needs.

The training time schedule is also indicated in Figure 1. The program is intended to last for ten weeks per instructions from the VITA staff. Although specialty courses 
are indicated to ruti concurrenlly, they may be scheduled to run at different times depending upon participant interest and consultation with the VITA staff.

\section{Orieritalion and Technical English (64 hours, 2 weeks)}

Most of the technicians and virtually all of the Afghan engineers working in the VITA program received their advanced training in English. However, these individuals received their educational training more than twelve years ago. Further, they may not have worked professionally using the English language for many years if at all. A working knowledge of technical English is considered essential for communicating with VITA, AID and relief organizations. This is needed for preparing reports, and to allow individuals to upgrade lechnical skills either formally or informally, and development of compuler skills. Therefore, the first element of the program will be a 32 hour refresher course in technical English. This training will be strengthened during the skills assessment and during more
formal classes.

The lechnical English review will focus on language areas that are required by the participants. Specifically, the course will include terminology used in technical drafting, surveying and mapping, calculators and calculations, weights and measures, and engineering symbols. This will include the English vocabulary and simple English styles and formats typically used in technical communications.

\section{Skills and Basic Assessment (64 hours, 2 weeks)}

This element of the program is intended to refresh the skills of all participants in basic communications, enginecring calculations and engineering measurements. During this element of the program, participants will be monitored individually to determine their existing skill levels and potential for additional training.

\section{Fundamentals of Engineering for Engineers (128 hours, 4 weeks)}

This element of the program is intended to refresh the basic engineering knowledge of B.Sc. level Civil Engineers. It will review most of the courses covered in the first year and half of engineering education. In practice, these courses provide the basic background for analysis and solution of engineering problems. Very often, the engineer must rely on, this information for the 'solution" of problems in the field.

\section{Fundamentals of Engineering for Technicians (128 hours, 1 weeks)}

This element is similar to that described above for engineers except that there will be more emphasis on practical approaches rather than mathematics. More time will be devoted to each topic and examples and problems will be very practical compared to engineering training described above. It is anticipated that most technicians will have previous training or equivalent practical experience. It is important that the technicians understand design and analysis concepts so they appreciate a design or can appropriately modify a design in the field, as necessary, to meet performance
expectations. 


\section{SPECIALTY COURSES}

As previously indicated, the purpose of the specialty courses is to upgrade the skills of VITA engineers and technicians serving the people of $\wedge$ fghanistan. Referring 10 Figure 1, specialty courses are scheduled for two weeks each, some courses are for engineers or technicians, and somie courses may be taken by participants from both
groups.

\section{Route Surveying}

This course is intended to upgrade the skills of technicians and engineers in surveying highways and bridges. Site reconnaissance and identification of suitable local malcrials for construction projects will also be discussed.

\section{Roadways and Bridges}

This course is intended to upgrade the skills of engineers in design of highways including drainage and bridges. Bridge applications will be based on existing VITA designs of vehicle and foot bridges.

Irrigation

This course is intended to upgrade the skills of engineers in designing water conveyance and irrigation struclures. Methods of estimating the irrigation water requirements for a region will also be discussed in terms of securing necessary water. resources. Applications will be to gravity-surface irrigation systems. 
Course Description
= = = = = = = = = = = = = = = $===0$

Orientation \& Technical

English (Two weeks, 64 hours)

Skill \& Basic Assessment two weeks (64 hours)
Proposed by CID

$==$

1. Technical Drafting:

a. Preparation of Engineerings drawings

b. Reading and interpreting engineering drawings

2. Calculator \& caiculations:

a. Use of Engineering calcularotr

b. Technical calculators

c. Percentage

d. Concept of accuracy, precision \& errors

3. Elementry surveying

a. Surveying instruments

b. Scales \& verniers

d. Dlstance angies \& elevatlons

4. Weights \& Measures

a. Weights, volumes, \& lengths

b. Materlal classifications sleve analysis

c. Accuracy, precision \& errors

d. Engineering symbols
1. Map \& Maping
2. Keyboarding, calculator \& computer symbols

Fundamentals of Englneering for engineers (128 hours, lour weeks)
Recommended by VITA

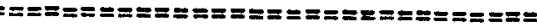

1. Technlcal Drafting:

a. Preparation of Engineering drawings

b. Reading and Interpreting engineering drawings

2. Caiculator \& caiculations:

a. Use of Englneering calculator

b. Concepls of accuracy

c. Practical problems

3. Units \& dimensions

a. Welghts, volumes, lengths etc.

b. Converslon

c. Scales

1. Mathematics

a. College Algebara

1. Algebralc varlables

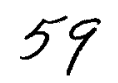

$\mathrm{H}-5 / 10$ 


\section{Solution of algebralc} equations

3. Formulation of algebraic problems

4. Quadratic \& cublc equalions

5. Simultaneous equations

6. Logarithms \& power functions

7. Examples \& problems

b. Trigonometry

1. Trigonometric functions

2. Trigonometric identities

3. Law of $\sin \theta s \& \operatorname{cosin} \theta s$

4. General plan trigonometry.

5. Exampies \& problems

c. Elementry calculus

1. Engineering application of Ilmits.

a. Scope of curve at a point

b. Area under acurve

2. Dlferentation of simple equation forms

3. Integration of simple equation forms.

4. Examples \& probiems

2. Engineering Mechanics

a. Statics

1. Forces moments and assoclated units

2. Resultant of a system of forces.

3. Resultant of moments

4. Equilibrlum of forces \& moments
2. Solution of algebralc equations

3. Formulation of algebralc problems

4. Quadratic equatlons

5. Simultaneous equations

6. Graphs \& curves

7. Examples \& problems

b. Trigonometry

1. Trigonometric functions

2. Trigonometrlc angles: Degrees, Radlans, Grads \& mills

3. Law of sines \& cosins

4. Examples \& problems

2. Engineering Mechanics

a. Statics

1. Forces moments and assoclated unlts

2. Resultant of a syslem of forces.

3. Resultant of moments

4. Examples \& problems 
5. Trusses \& cabies

6. Friction

7. Examples \& problems

b. Dynamics

1. Position, velocity \& acceleration

2. Partical motion

3. Moment of inertia

4. Rigid body motion

5. Work \& energy method

6. Impules \& momentum

7. Elementry vibrations

8. Examples \& problems

c. Mechanic of materials

1. Concepts of stress \& strain

2. Torsion

3. Analysis of beams

a. Moment of Inertia

b. Shear \& moment dlagrams

c. Flexure of beams

d. Shear In beams

e. Restrained beams

f. Peinforced concrete beams

4. Alveted \& welded connection

5. Columns

a. Critical loads

b. Evier equation

c. Exmirlcal formula

c. Mechanlc of materials

1. Concepts of stress \& strain

2. Torsion

3. Analysis of beams

a. Moment of Inertia

b. Shear \& moment diagrams

c. Flexure of beams

d. Shear In beams

e. Restralned beams

f. Reinforced concrete beams

$$
61
$$


The Route Surveying is here Included for Tngineers, part of "Fundementals", rather than a separale short course $\gg$
Fundamentals of Engineering for Techniclans (128 hours, four weeks)
6. Inelastic action

a. Limit moment \& torque

b. Residual stresses

7. Impact loading

8. Examples \& problems

d. Fluid Mechanic (Emphasis on water)

1. Properties of fluids

2. Fluid stallics

a. Pressure

b. Hydrustatic force on area

3. Fluid flow

a. Energy \& momentum

b. Measurement of fluld flow

4. Flow in plpe

5. Open chane flow

6. Pumps

7. Examples \& problems

\section{Mathematics}

a. Basic Mathematics operation, areas, \& volume

b. Basic algebra

1. Algebralc varlations
4. Impact loading
5. Example \& problems

d. Fluld Mechanic (Emphasis on water)

1. Fluid flow

a. Energy \& momentum

b. Measurement of fluld flow

2. Open chane flow

3. Examples \& problems

e. Houte surveying

1. Basic definttion

2. Geometry \& type of sample horlzontal covers

3. Design methods of curves

4. Sectlons \& volume of earth work

5. Cost estimation

1. Baslc mathematics

a. Baslc mathematics operatlons, areas, volumes, \& percentage 
2. Solutions of algebraic equations

c. Trigonometry

1. Definition of trigonometric functions

2. Right angle trlgonometry

d. Appilcations and problems

2. Engineering mechanics

a. Force \& moments

1. Definillon of force \& moments

2. Resultants \& equilibrium moments

3. Estimation resultant forces.

4. Applicallons \& probiems

b. Dynamic

1. Linear motion, velocity \& acceleration

2. Impact

3. Appilcation \& problems

c. Mechanics of materlais

1. Concepts of stress \& strain

2. Torsion \& Flexure of regular shaps

3. Use of tabular information for team design

4. Columns, critical loads, empirical solutions

5. Alveted \& welded connecllons

6. Applications \& problems

d. Fluid mechanics (water)

1. Fluid pressure

2. Pressure on areas b. Baslc algebra

1. Algebraic varlations

2. Solutions of algebralc equations

c. Trigonometry

1. Definttion of trigonometrlc functions

2. Right angie trigonometry

d. Applications and problems

2. Statics

a. Force \& moments

1. Definition of force \& moments

2. Resultants \& equillbrium moments

3. Estimation resultant forces.

4. Applications \& problems

c. Materials of constructions.

1. General

2. Stones

3. Brlck

4. Soil as construction materials

5. Agregale

6. Cement, llme \& mortar

7. Concrete

8. Timber

9. Steel

10. Gablon

d. Hydraullcs 


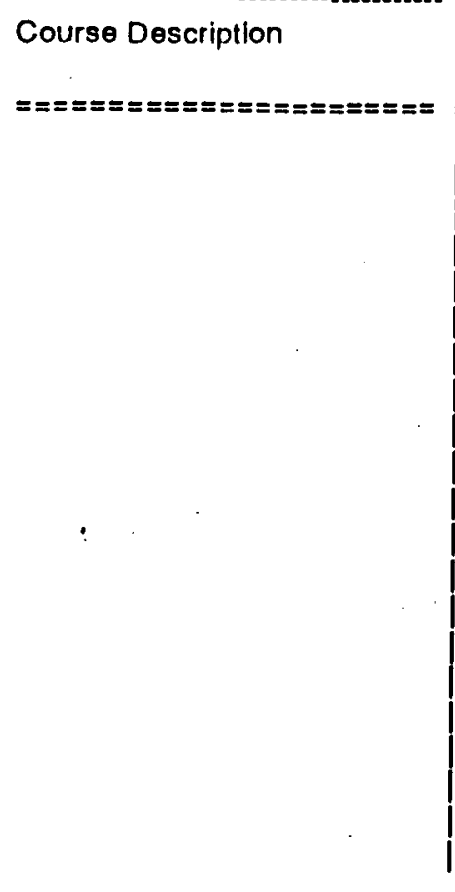

Proposed by CID

Recommended by VITA

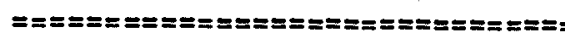

3. Fluld flow

4. Flow In plpe, tabular solutions

5. Open channel flow, fabular sulution

6. Pumps

7. Application \& problems

e. Elements of surveying

1. Horizontal measurements

2. Angles and direction

3. Vertical measurement

4. Examples \& problems
1. Fiuld flow

2. Open channel flow \& tabular solutions

3. Flow measurement

4. Appllcalions \& problems

e. Elements of surveying

1. Horizontal measurements

2. Angles and direction

3. Vertical measurement

4. Examples \& problems

SHORT COURSES - two weoks

A. Irrigation (for Engineers)

Irrigation

1. Design of earthen canals and related structures

2. Design of Irrigatlon water systems

3. River meandering and control

B. Roadways and Bridges (for Englneers)

as proposed

C. Project Management (for Techniclans)

as proposed

D. Cost Estimating (for Techniclans)

as proposed 
(iEINERAL DATA

ARS Nmbr/Name:

Subproj Nmbr:

Village:

District:

Subproject Type: Irrigation

Start date:

Rds \& Brdg

Commander:

Subtype:

Compl date:

Tanzeem:

Budget: Cash:

Fertilizer:

Food:

T'otal (Rs):

Monitor:

Inspect Date:

PLANNING

Was a subproject request form completed?

Was a feasibility study conducted?

Was a subproject planning form completed?

Was Form $1 \mathrm{R}-1$ or $\mathrm{RB}-1$ completed?

Was a Shura involved in planning/approval?

Was there consensus among local authorities/community that this subproject should be accomplished before other requested projects that were not approved or deferred?

IMPIEMENT'ATION

Have any umusual problems (technical or otherwise) been encountered in the construction of the subproject?

Were timely measures taken to solve these problems?

Describe any outstanding problems that have prevented or will prevent a successful outcome of the subproject. 
OVERALL IMPACT

i/pon completion of the construction work, will the subproject facility be fully functional?

In your opinion, will the subproject have a significant impact on agricultural production?

Has there been an adverse social impact? If so, explain.

Lloes the information appearing on Form IR-1 or RB-1

seem to be approximately correct, with regard to

benefits that will be provided by the subproject:

IR-1: $\quad$ Total farm families served?

Workers employed?

Crops irrigated?

Total jeribs irrigated?

$\mathrm{RB}-1$ : Towns connected?

Workers employed?

Jeribs cultivated within

road influence area?

Total families within road influence area?

CONRIUNITY PARTICIPATION

Have there been any problems in recruiting workers?

Vid community members request the subproject?

Have any labor or materials been contributed.

Has the community provided project staff with any other assistance (e.g., security)?

LABOR PAYMENISS

Are payments made directly to workers by ARR staff?

Are the workers actually receiving full payments, whether in cash, food or fertilizer? 


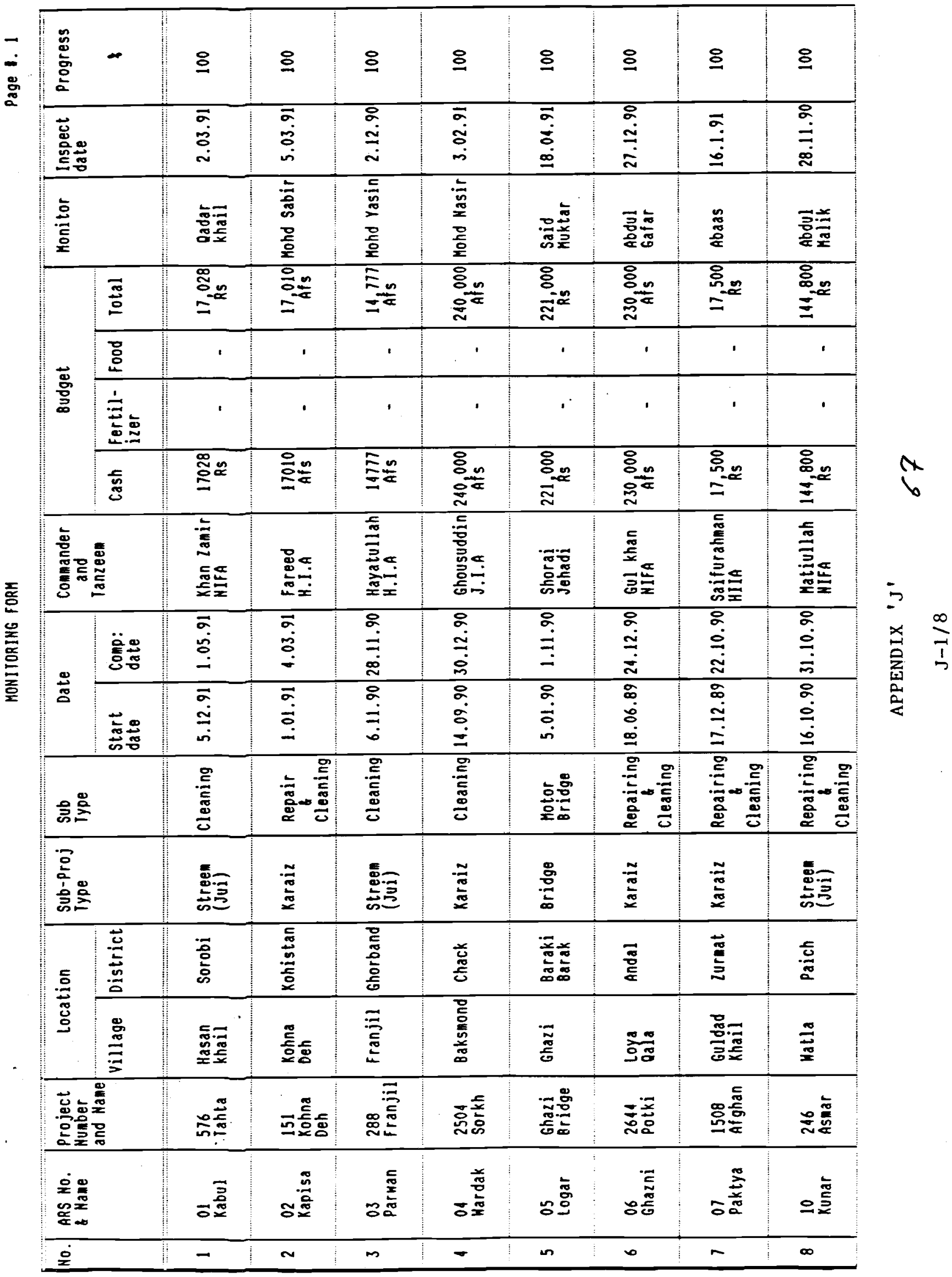




\begin{tabular}{|c|c|c|c|c|c|c|c|c|}
\hline 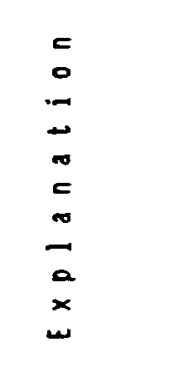 & & & & & & & & \\
\hline 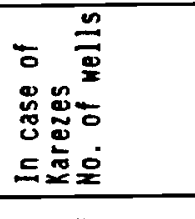 & ' & $\simeq$ & & $\bar{\sim}$ & & $\therefore$ & & \\
\hline 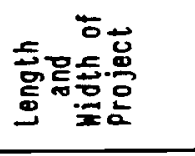 & & is & & $\begin{array}{l}-5 \\
\$ 8 \\
\$ 8\end{array}$ & $\stackrel{-50}{\pi}$ & 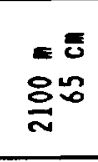 & $\stackrel{E}{\circ}$ & : \\
\hline 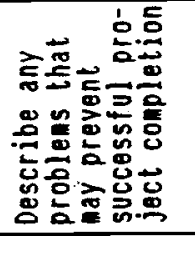 & 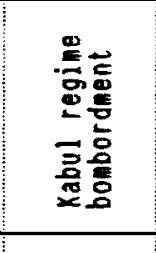 & ' & & & ' & ' & & ' \\
\hline 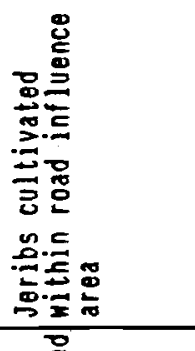 & & ' & ' & ' & . & . & ' & ' \\
\hline 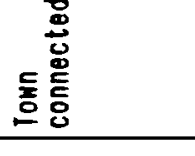 & & ' & ' & 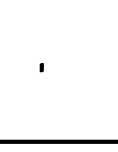 & ' & . & & ' \\
\hline 罢 & $\therefore$ & 8 & ঃ & 8 & ' & $\stackrel{8}{\circ}$ & $\cong$ & 品 \\
\hline 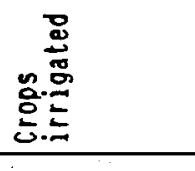 & 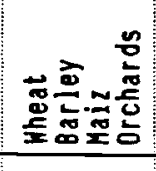 & 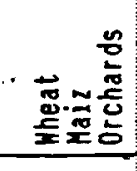 & 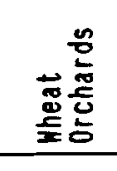 & 譬产 & . & 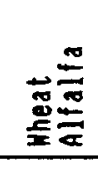 & 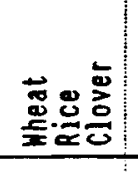 & 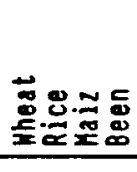 \\
\hline 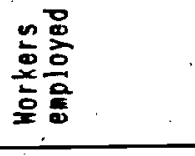 & 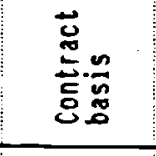 & $=$ & . & $=$ & & . & . & . \\
\hline$\dot{\dot{x}}$ & - & $\sim$ & $m$ & - & $n$ & $\circ$ & - & $\infty$ \\
\hline
\end{tabular}




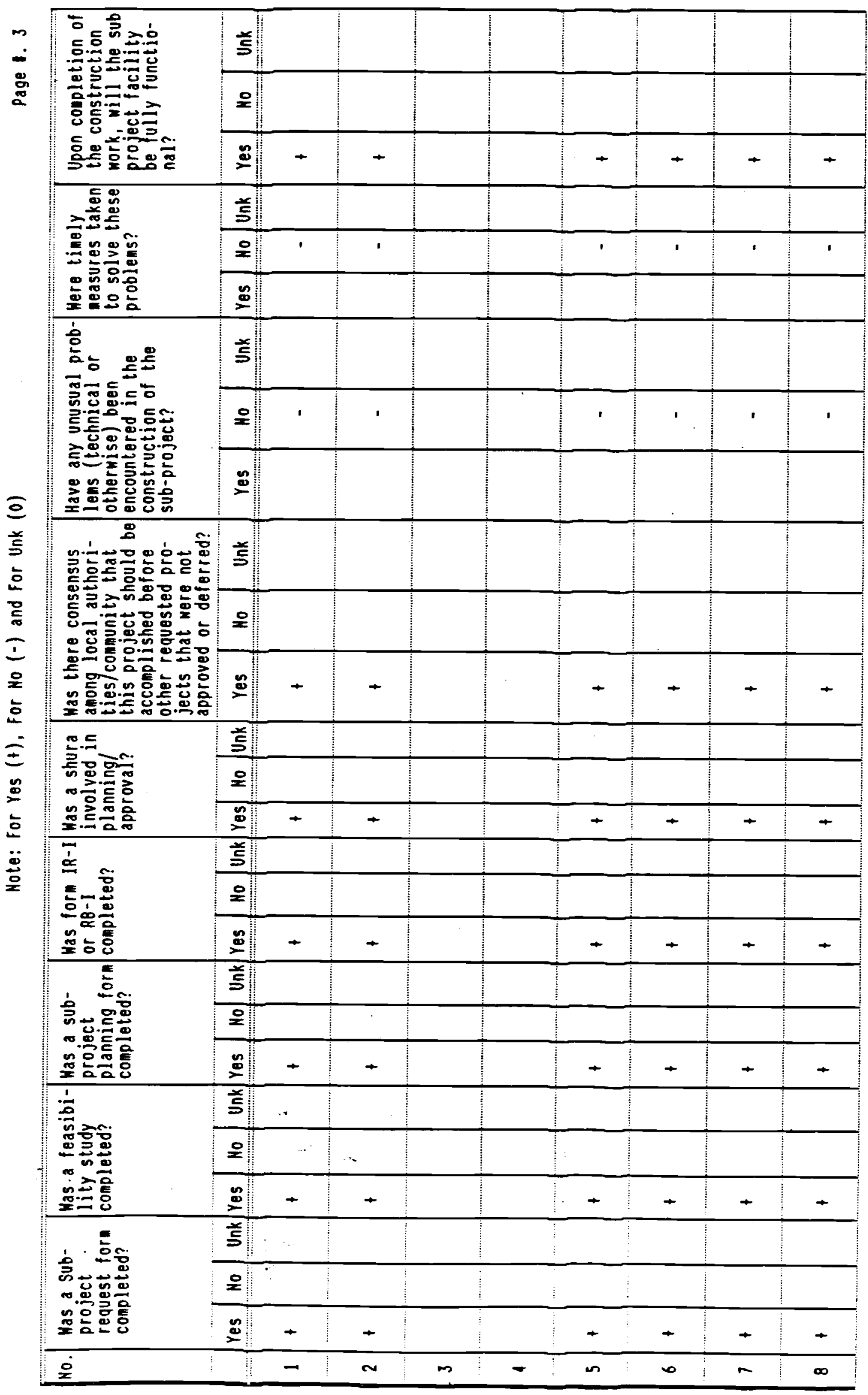




\begin{tabular}{|c|c|c|c|c|c|c|c|c|c|}
\hline $\begin{array}{l}\tilde{\alpha} \\
\vdots \\
\vdots \\
0 \\
\vdots\end{array}$ & - & 묘 & 음 & & & 임 & 으 & $\stackrel{\sim}{\sim}$ & 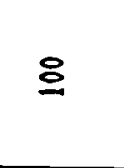 \\
\hline 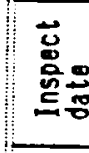 & & $\begin{array}{l}8 \\
\check{\check{1}} \\
\dot{2}\end{array}$ & $\begin{array}{l}\stackrel{2}{1} \\
\dot{m}\end{array}$ & & & $\begin{array}{l}8 \\
0 \\
0 \\
0\end{array}$ & a & $\begin{array}{l}9 \\
\vdots \\
\vdots\end{array}$ & $\begin{array}{l}\ddot{a} \\
\ddot{0} \\
\dot{0}\end{array}$ \\
\hline 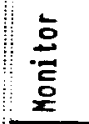 & & 을 & 몽융 & & & 흫홍 & 홓형 & 뫃치 & 可: \\
\hline \multirow{4}{*}{ 票 } & $\overrightarrow{\overrightarrow{0}}$ & $\begin{array}{l}\text { 8. } \\
8 \\
8 \\
8\end{array}$ & $\frac{8}{\frac{8}{6}}$ & & & 造电 & $\mathrm{O}^{-\infty}$ & 용 & $\frac{0}{a} a^{-\infty}$ \\
\hline & 8 & ' & ' & & . & $=$ & ' & ' & ' \\
\hline & $\begin{array}{l}\therefore \pm \\
\dot{\Xi}=\end{array}$ & ' & ' & & & ' & ' & ' & ' \\
\hline & 䍃 & 豆脶 & $\frac{8}{8}$ & & & 总出 & ${ }_{\text {옹 }} \infty$ & ㅎㅎㅎ용 & $\frac{0}{\alpha} \approx$ \\
\hline 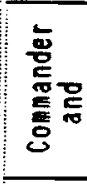 & & 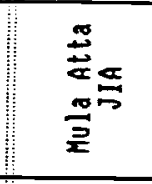 & 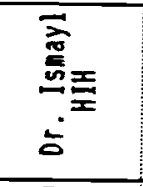 & & & 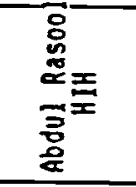 & 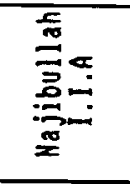 & 흥 & 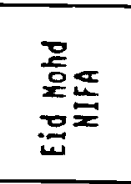 \\
\hline \multirow{2}{*}{$\stackrel{\infty}{=}$} & 关思 & ' & $\begin{array}{l}\dot{0} \\
\dot{0} \\
\approx\end{array}$ & & & $\begin{array}{l}8 \\
8 \\
0 \\
0 \\
0 \\
\end{array}$ & $\begin{array}{l}9 \\
\dddot{\dddot{m}} \\
\dot{m}\end{array}$ & ' & $\begin{array}{l}\stackrel{8}{0} \\
0 \\
0 \\
0\end{array}$ \\
\hline & 吾恋 & $\begin{array}{l}2 \\
\vdots \\
\vdots \\
\vdots\end{array}$ & $\begin{array}{l}8 \\
8 \\
8 \\
\end{array}$ & & & $\begin{array}{l}9 \\
\dot{0} \\
\dot{0} \\
0\end{array}$ & $\begin{array}{l}8 \\
\dddot{a} \\
\approx\end{array}$ & 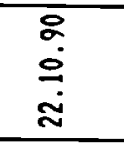 & $\begin{array}{l}8 \\
0 \\
0 \\
0\end{array}$ \\
\hline \multicolumn{2}{|c|}{ 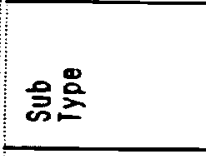 } & 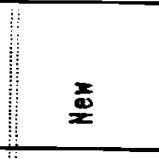 & 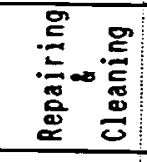 & & & 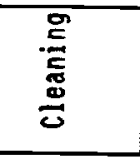 & 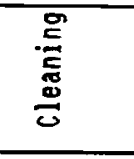 & $\stackrel{\bar{\Xi}}{\frac{\pi}{0}}$ & 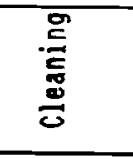 \\
\hline \multicolumn{2}{|c|}{ 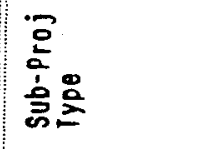 } & $\begin{array}{l}\square \\
0 \\
0\end{array}$ & $\begin{array}{l}\bar{\pi} \\
\mathbb{5} \\
\end{array}$ & & & 竧焉 & 言离 & $\begin{array}{l}\text { 웅 } \\
0\end{array}$ & 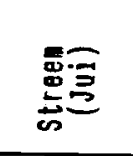 \\
\hline \multirow{2}{*}{ 总 } & 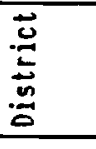 & 竞 & 咅言 & & & 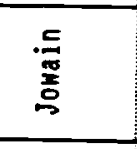 & 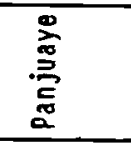 & 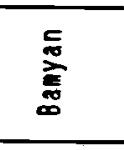 & $\begin{array}{l}\overrightarrow{7} \\
\bar{E} \\
0\end{array}$ \\
\hline & $\stackrel{\Xi}{\Xi}$ & 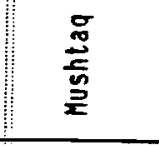 & 홍옹 & & & 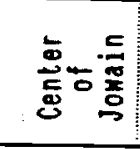 & : & 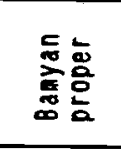 & 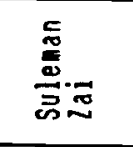 \\
\hline \multicolumn{2}{|c|}{ 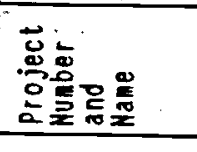 } & 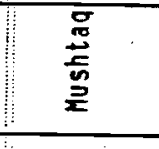 & 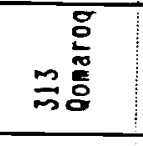 & 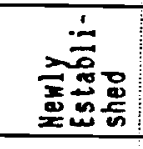 & 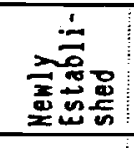 & 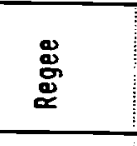 & $\begin{aligned} & \frac{2}{2} \\
& \approx \stackrel{\circ}{\circ} \\
&=\end{aligned}$ & : & ミ总 \\
\hline \multicolumn{2}{|l|}{ 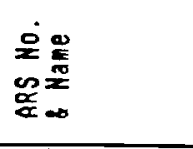 } & 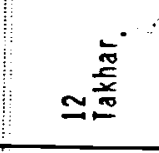 & 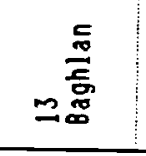 & $=\frac{\tilde{z}}{\underline{z}}$ & 유히호 & $\approx \stackrel{5}{\frac{5}{5}}$ & ন & $\underset{\infty}{\infty}$ & 紊 \\
\hline \multicolumn{2}{|l|}{$\dot{0}$} & $a$ & 으 & $\equiv$ & $\simeq$ & 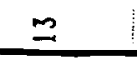 & $\equiv$ & $\cong$ & 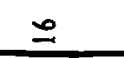 \\
\hline
\end{tabular}




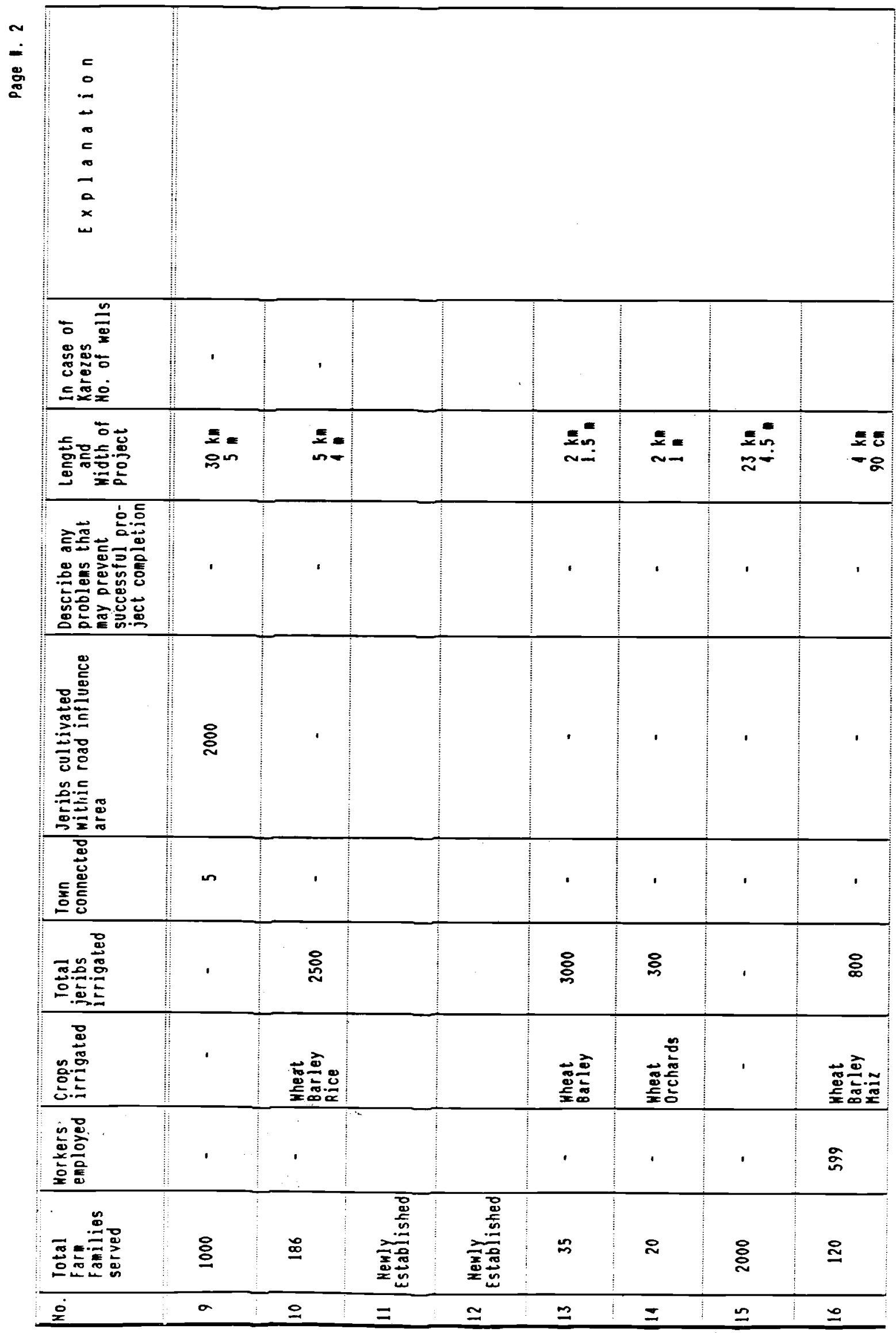




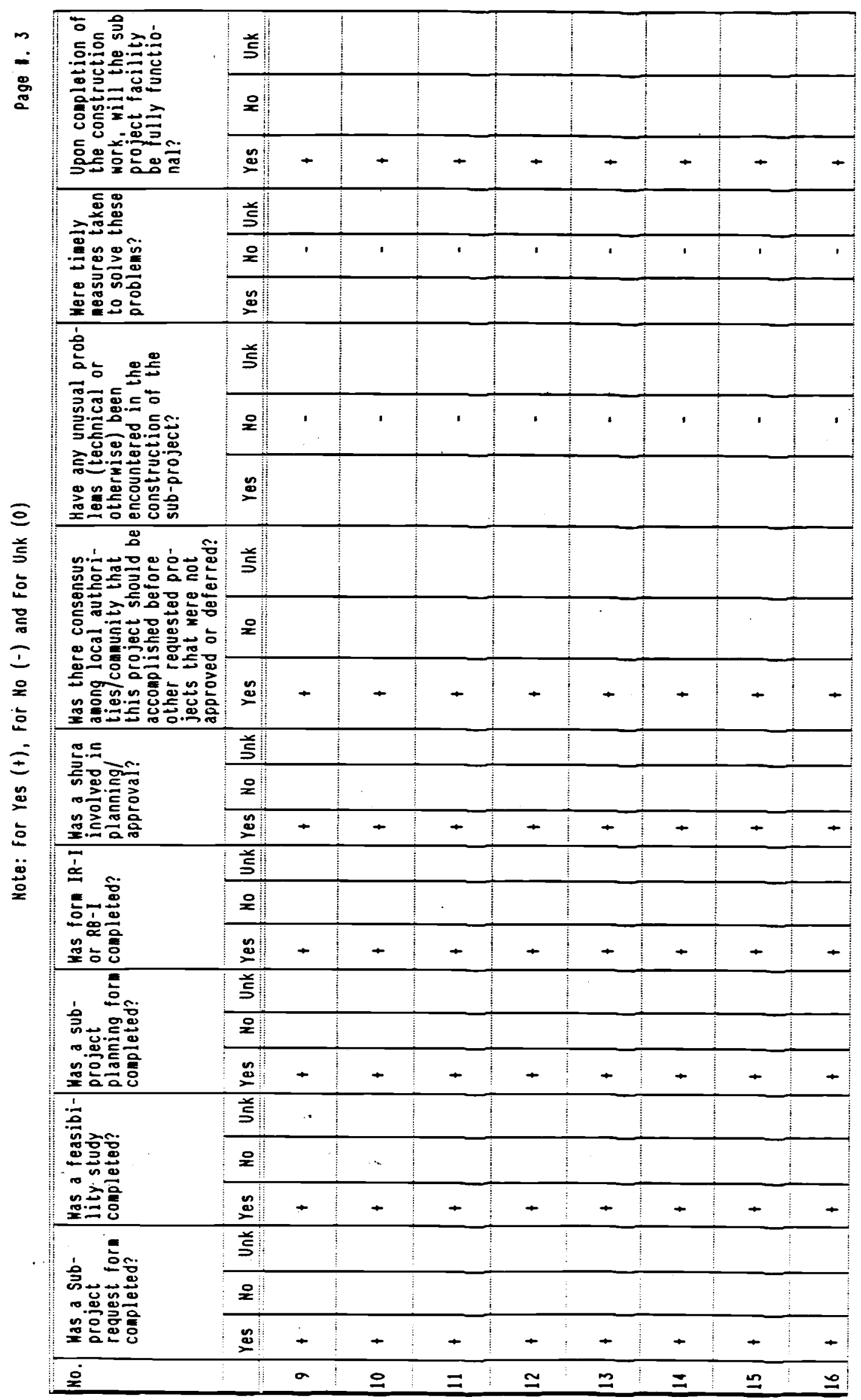

$N \frac{\infty}{i}$ 


\begin{tabular}{|c|c|c|c|c|c|c|c|c|c|}
\hline 交立. & 节: & & & & & & & & \\
\hline 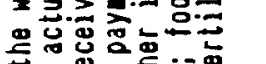 & 웆 & & & & & & & & \\
\hline 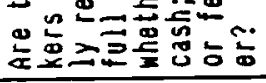 & 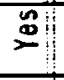 & - & - & + & + & + & - & + & - \\
\hline $\begin{array}{c}9 \\
\geq \infty\end{array}$ & 总 & & & & & & & & \\
\hline 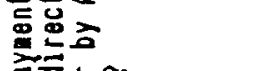 & 우 & ' & , & ' & . & ' & ' & ' & ' \\
\hline 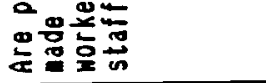 & $\stackrel{\infty}{\infty}$ & & & & & & & & \\
\hline 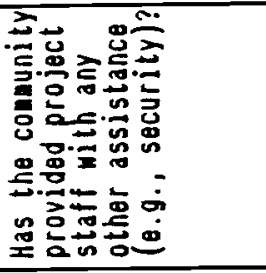 & $\begin{array}{l} \\
\text { 우 } \\
\text { ' } \\
\boldsymbol{\Phi}\end{array}$ & 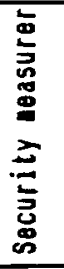 & 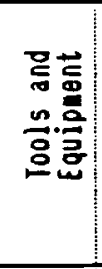 & 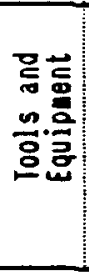 & 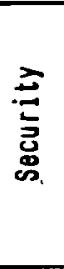 & $\cdot$ & 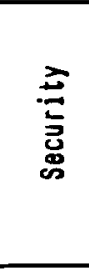 & 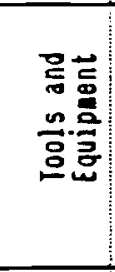 & - \\
\hline 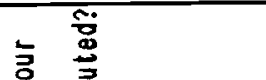 & 兰: & $\because$ & & & & & & & \\
\hline 至言 & 운 & & & & & ' & & & \\
\hline 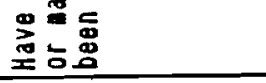 & $\stackrel{\boldsymbol{\Phi}}{\boldsymbol{=}}$ & - & - & + & + & & - & - & - \\
\hline 至 & 兰 & & & & & & & & \\
\hline 爱怘 & 오 & & & & & & & & \\
\hline 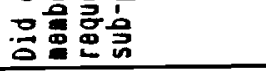 & $\stackrel{\infty}{\infty}$ & + & + & + & + & + & - & - & - \\
\hline 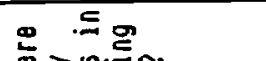 & 兰 & & & & & & & & \\
\hline 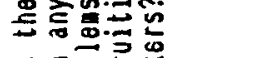 & 웆 & . & , & . & ' & ' & ' & ' & ' \\
\hline 产㐓范灾兑 & $\stackrel{\infty}{\infty}$ & & & & & & & & \\
\hline 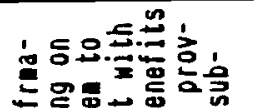 & $\stackrel{-}{5}$ & & & & 0 & & & & \\
\hline 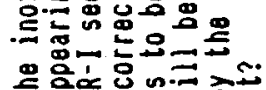 & 유 & & & & & & & & \\
\hline 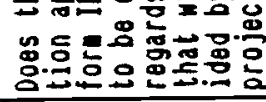 & $\stackrel{\infty}{\infty}$ & - & - & - & & - & + & - & - \\
\hline 医 & 兰 & & & & & & & & \\
\hline 心 & 우 & · & ' & ' & ' & - & ' & , & ' \\
\hline 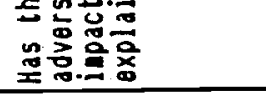 & $\stackrel{\boldsymbol{m}}{\mathbf{\infty}}$ & & $=$ & & & & & & \\
\hline 岕营苛 & 总 & & & & & & & & \\
\hline 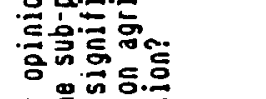 & 요 & & & & & & & & \\
\hline 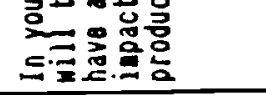 & $\stackrel{\boldsymbol{\Phi}}{\boldsymbol{\infty}}$ & - & $\rightarrow$ & - & - & $\rightarrow$ & $\rightarrow$ & + & - \\
\hline$=$ & 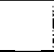 & $a$ & 으 & $=$ & $\simeq$ & $=$ & $=$ & $=$ & 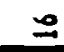 \\
\hline
\end{tabular}




\begin{tabular}{|c|c|c|c|c|c|}
\hline \multicolumn{6}{|c|}{$\begin{array}{l}\text { LEVEL-1 MONITORING } \\
\text { SUB-PROJECTS/INSPECTED } \\
\text { (JUNE 1990-APRIL 1991) }\end{array}$} \\
\hline NO & PROVINCE & IRRIGATION & $\begin{array}{c}\text { BRIDGES \& } \\
\text { ROADS }\end{array}$ & TOTAL & REMARKS \\
\hline 01 & Kabul & 43 & --- & 43 & \\
\hline 02 & Kapisa & 23 & 6 & 29 & \\
\hline 03 & Parwan & 46 & 6 & 52 & \\
\hline 04 & Wardak & 129 &.-- & 129 & \\
\hline 05 & Logar & 175 & 1 & 176 & \\
\hline 06 & Ghazni & 58 & -- & 58 & \\
\hline 07 & Paktia & 142 & -- & 142 & \\
\hline 10 & Konar & 49 & 11 & 60 & \\
\hline 12 & Takhar & 1 & 1 & 2 & \\
\hline 13 & Baghlan & --- & --- & --- & New ARS \\
\hline 14 & Kundoz & --- & --- & --- & New ARS \\
\hline 20 & Herat & --- & --- & --- & New ARS \\
\hline 21 & Farah & 40 & --- & 40 & \\
\hline 24 & Kandahar & 23 & --- & 23 & \\
\hline 28 & Bamyan & 13 & 1 & 14 & \\
\hline 29 & Paktika & 138 & $-\cdots$ & 138 & \\
\hline \multicolumn{6}{|l|}{. } \\
\hline \multicolumn{2}{|c|}{ TOTAL } & 880 & 26 & 906 & \\
\hline
\end{tabular}


Level -3 Monitoring

(Sub-projects Inspected April, 1990 - March, 1991)

\begin{tabular}{|c|c|c|c|c|c|}
\hline $\begin{array}{l}\text { No. of } \\
\text { Project }\end{array}$ & Province & Irrigation & $\begin{array}{l}\text { Bridges and } \\
\text { Roads }\end{array}$ & Total & Remarks \\
\hline 01 & Kabul & 43 & 1 & 44 & \\
\hline 02 & Kapisa & 23 & - & 23 & \\
\hline 03 & Parwan & 46 & 1 & 47 & \\
\hline 04 & Wardak & 129 & 4 & 133 & \\
\hline 05 & Logar & 175 & 2 & 177 & \\
\hline 06 & Ghazni & 58 & - & 58 & \\
\hline 07 & Paktia & 142 & 2 & 144 & \\
\hline 10 & Kunar & 49 & 3 & 52 & \\
\hline 12 & Takhar & 1 & 3 & 4 & \\
\hline 13 & Baghlan & - & - & - & \\
\hline 14 & Kunduz & - & - & - & New ARS \\
\hline 20 & Herat & - & - & - & New ARS \\
\hline 21 & Farah & 40 & - & 40 & \\
\hline 24 & Kandahar & 23 & 1 & 24 & \\
\hline 28 & Bamyan & 13 & 1 & 14 & \\
\hline 29 & Paktika & 138 & - & 138 & \\
\hline & Total: & 880 & 18 & 898 & \\
\hline
\end{tabular}


PARWAN

: in

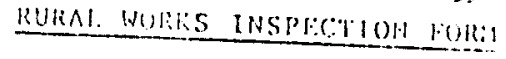

Lutc Insperted

inspected isy

$$
\begin{aligned}
& \text { : } 25.12 .90 \\
& \text { : } \\
& \text { Eng. M. Ibrahim i } \\
& \text { Ghorband and Fandaqistan Roads } \\
& : \quad \text { : 03-0302-RD-02-68 and 72: } \\
& 4.7 .89 \\
& \text { September, } 1990
\end{aligned}
$$

iroject lame

rroject fumber

Date Project Surveyed

Datc Project Limpicmentation

scarted

Objective of Inspection

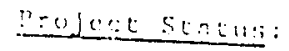

a. Percentage completed

E. Work Stoppert \& Reason

$92 \%$ and $70 \%$

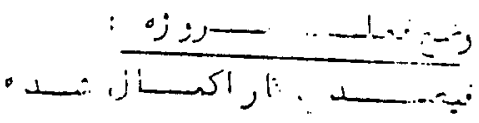

is:

Due to shortage of some professional workers.

Due to weather or climate which was snowing for three months

inbour on $5120:$

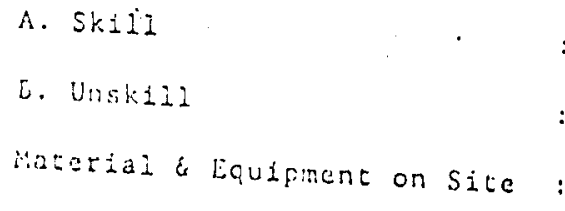

A. Skili

L. Unskili

Aasarial \& Equpinent on Site:

Gonstruction ?'ogtess

$: \frac{\text { Some exists }}{\text { Exists }}$ Some exists $:$

$92 \%$ and $70 \%$

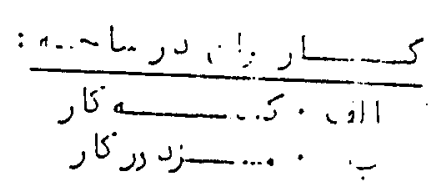

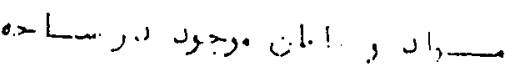

نLi- $L$ L tri,

Conscruction probien

Shortage of personnel, climate problems.

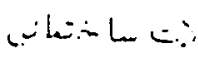


or Sysiea Operation

OS System Hes Development

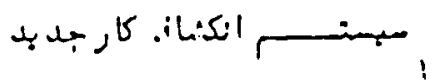

RA:InB CIAdCS:

Excellent 4 
Date Insperted Inspected by

Project Name

Project Number

Date Project Surveyed

Date Project Lmplementation Staried

Objective of Inspection
:

\begin{tabular}{l}
$\frac{18.1 .91}{\text { Eng. Najibullah }}$ \\
\hline Eng. Sardar Mohammad \\
\hline
\end{tabular}

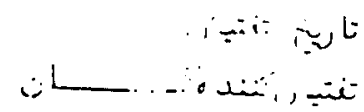

jos......

שיח

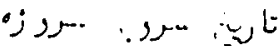

t

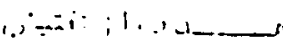
according to the maps and guiding the technical personnel.

Projectesentus:

A. Perceniage Completed

B. Work Scopped \& Rason'
$30 \%$

Some security problems Climate problems

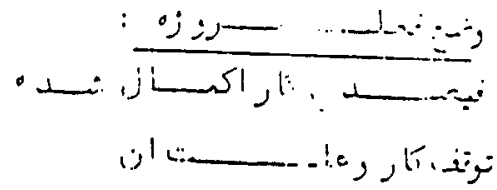

Liabour on site:

A. $\operatorname{ski11}$

E. Unski11

Matc:ial \& Equipent on Sice:

Corstruesion progress

1
Exists

Exists

Some exists

$30 \%$

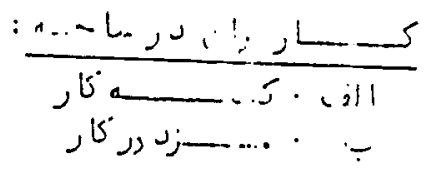

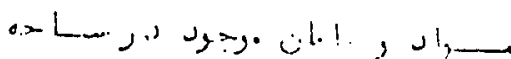

بين.

1. Shortage of some equipment:

2. Shortage of some technical workers whil L 
"irmenil $\frac{\text { 1. Because they prepare their material and equipment }}{\text { from Peshawar, therefore it takes time to provide them:- }}$

\section{Shortage of professional personnel.}

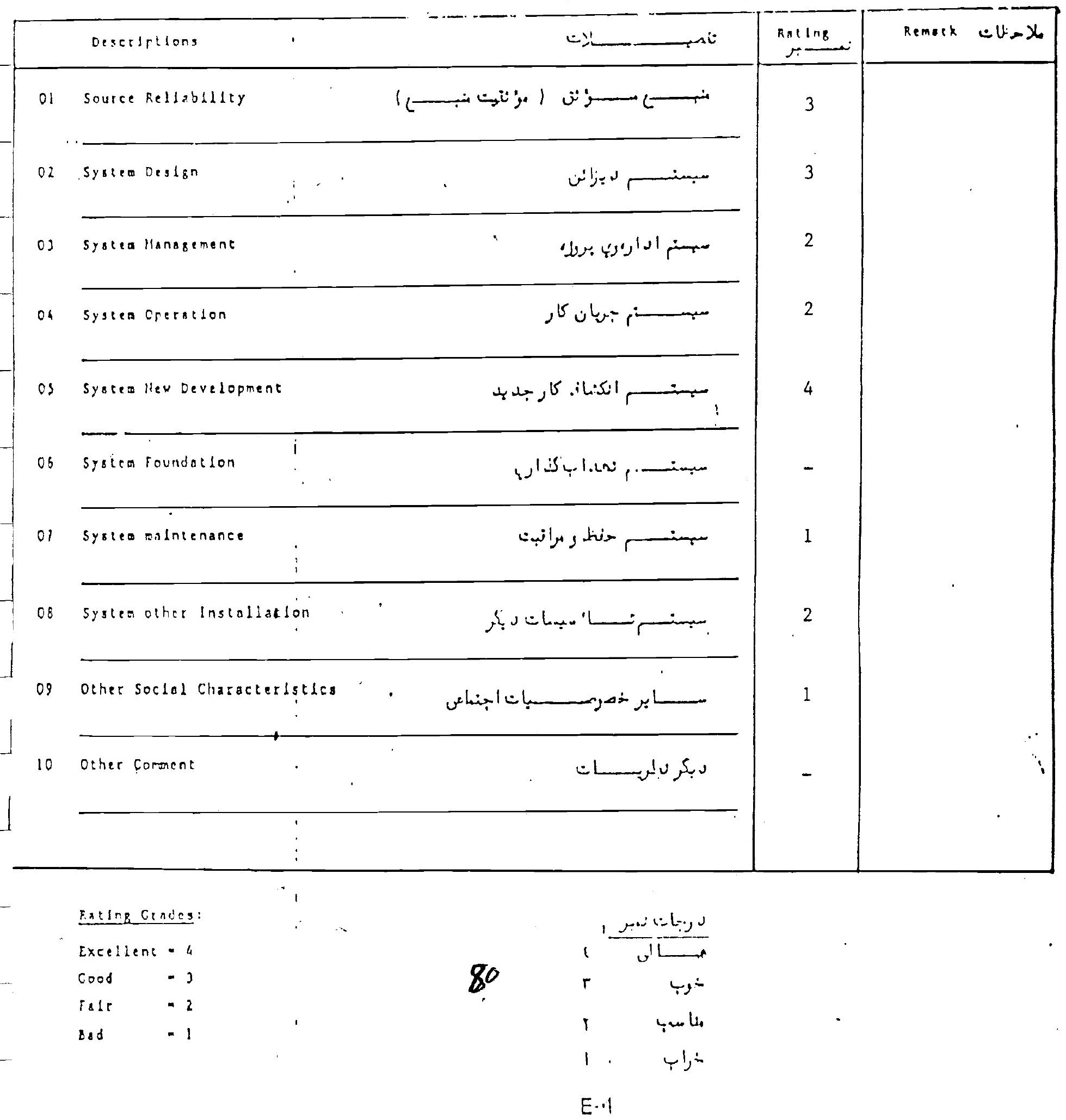


Dite inspected

Inspected by

Project Viame

Project Number

Date project Surveyed

Date project lapleticntation

seatece

Cbjective of Insicetion
$: \quad 15.1 .91$

Eng. M. Aziz Salehi

Eng. Sardar Mohammad

Tech. M. Nader

$: \frac{\text { Naray Bridge }}{27.5 .89}:$

7.10 .90

To inspect whether work is beịng done

on the project according to the maps and guide the professional personnel in the project.

Projectestincu:s:

A Percencage conpleted

3. Work.stopped \& Reason'

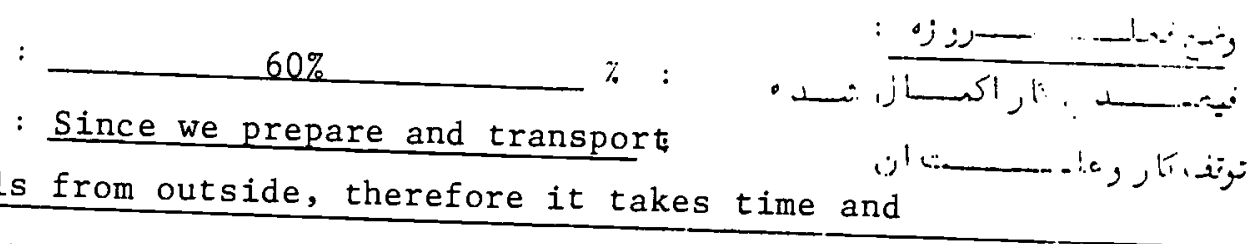

some materials from outside, therefore it takes time and

does not reach on proper time in the field.

abour on S1:2: :

\section{A. Sriil}

B. Unsk12i

Haterial \& Equipment on Sice :

conscruation l'rogress

$60 \%$

Masons, Technicians, Carpenter and Engineer were working jointly

to complete the construction works.
Exists

Exists

Partially, some of them

not exist.

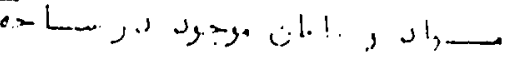

Oolisäuctan Problen

1. Because the cable was going to be prepared from Lahore by RoNCo, it took
long time to be prepared.

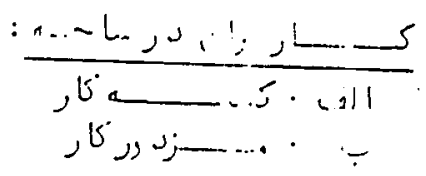

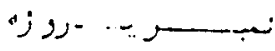

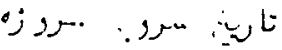
E

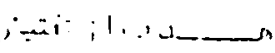
$i=i-i t$ $\therefore$ L . . . . ;

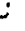



cement, steel bars and other equipment).

2. Lack of machines and other equipments.

Desertetions

ビ

$\operatorname{Rat} \operatorname{lng} 8$

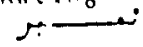

Remark

ol Source Rellabllty

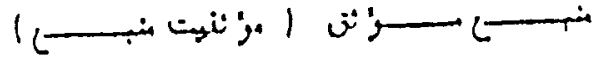

02 Systeo Des $18 n$

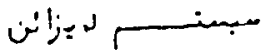

of Systen Operation

سمبس

Os Syoter lies Development

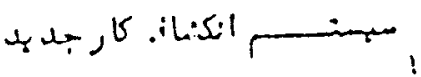

os system toundation

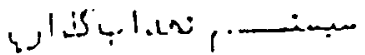

01 Systeo Dabutenance

عبهن

Q8 System other Installation

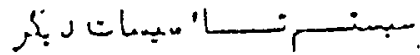

RatInB Gindes:

Excellens- 4

$\operatorname{cood}-3$

Falt $\quad 2$

Dad -1

\begin{tabular}{|c|c|}
\hline \multicolumn{2}{|c|}{ נ } \\
\hline 1 & ب \\
\hline$\tau$ & - \\
\hline 1 & ملاسب \\
\hline 1 & $+1,=$ \\
\hline & \\
\hline
\end{tabular}




\section{BTATEMENT OF WORK}

The following statement of work has been submitted by the Reconstruction Group of the center for International studies of the Massachusetts Institute of Technology (MIT) in response to a request for a proposal from the Volunteers in Technical Assistance (VITA) to conduct a feasibility study for establishing an independent Afghan-managed rural works entity.

Section 1 of this statement describes the subcontracting organization. Section 2 describes the tasks of the proposed feasibility study. Section 3 describes the methodology of the proposed feasibility study. Section 4 provides a description of the administrative staffing and schedule for the proposed study. section 5 provides a list of names of the consultants for the proposed team.

\section{SECTION 1 -- The subcontracting Group}

This statement of work has been submitted by members of the Workshop on Reconstruction in the Middle East, a group located within the Center for International studies (CIS) of the Massachusetts Institute of Technology. The Center for International studies, established in 1951, is the principal research center within MIT in the field of international and comparative studies. Its senior staff consists of forty faculty members drawn from various departments at MIT; it includes engineers, urban planners, historians, political scientists, and economists.

CIS has a long history and a record of research and applied projects in developing countries, in Latin America, sub-Sahara Africa, Southeast Asia, East Asia, the Middle East, and south Asia. The Reconstruction Group, is concerned with understanding the processes and problems associated with post-war reconstruction in the Middle East, broadly defined to include the region from Egypt to Afghanistan. Attention has been given to the role of international financial institutions, especially the World Bank and the International Monetary Fund, in large scale reconstruction, as well as to the role of local financial resources provided by governments, institutions, private entrepreneurs, and people of the region. The group has been concerned with the problems of relocating populations, rebuilding depleted skills and indigenous capabilities, assessing internal and external investment strategies in light of construction needs, evaluating appropriate modes of management of reconstruction activities, and exploring alternative modes of governance for communities undergoing reconstruction.

The Group is made up of faculty representing various disciplines such as civil engineering, urban and regional planning, political science, history, and architecture. Participants also include 
scholars in anthropology, economics, and psychology. This interdisciplinary mix has provided the group with an awareness of the complex interaction of the political, social, economic, and physical planning aspects of efforts to reconstruct war-torn regions. Members of the Reconstruction Group have had considerable field experience as consultants to governments and private agencies, conducting feasibility studies, evaluating programs and institutions, proposing designs for new institutions, establishing training programs, and developing project proposals.

\section{SECTION 2 -- Tasks of the Feasibility study}

VITA and USAID are concerned with how a program for agricultural and rural rehabilitation in Afghanistan, functioning out of Peshawar and Quetta with field offices working with local authorities within Afghanistan to repair and rehabilitate irrigation systems, roads, and bridges, can be evolved into an Afghan entity "capable of broadening its funding base and expanding its impact on rural infrastructure." The Reconstruction Group will assess the feasibility of this objective, first by considering the local, national, and international environment within which such an entity would function, and second by preparing a detailed set of recommendations for the establishment of such an entity.

An effective Afghan entity needs to be able to work with local authorities to carry out reconstruction and development activities. These activities must be able to be effectively monitored by donor agencies. Finally, the entity must have the standing or legitimacy to warrant support from a variety of bilateral and multilateral donors.

These tasks are made unusually difficult by the special political circumstances under which such an entity must function. Afghanistan's war has not yet ended, although the VITA program is within provinces considered peaceful. More fundamentally, the Agricultural Rehabilitation and Reconstruction (ARR) schemes function without the approval of Afghanistan's central government and the proposed Afghan entity may need to function independent of any central authority. Moreover, the tasks of the field offices have been limited primarily to the rehabilitation of irrigation systems, roads, and bridges and in the past have been intentionally kept small so as to minimize military attacks.

Taking all of these circumstances into account the present feasibility study will pay special attention to the following issues:

What responsibilities should be assigned to the proposed Afghan entity? Should it continue to be primarily concerned with rural public works or should it take on broader functions to enhance agricultural productivity and nurture small scale enterprises including agro-businesses? Given the 
present political situation within Afghanistan as well as the managerial and technical capabilities of the existing staff, what would be a realistic set of tasks for the proposed entity? A related question is whether the proposed entity should be a non-revenue generating institution dependent upon external support or whether it is feasible to take on activities that would provide some self-generating revenue.

Should the mandate of the proposed Afghan entity be the reconstruction of war-torn areas to which refugees are or will be returning, or should it also focus on the development of regions in which there has been little destruction but which have a high potential for agricultural productivity? What criteria should be used for selecting sites for assistance?

- Can the proposed entity engage in province-wide planning in the present political context or should the interactions be primarily if not exclusively with local institutions? How can the proposed entity most effectively interact with local and provincial authorities?

- What alternative institutional arrangements are possible? A private construction firm contracted to do public works? A non- profit quasi-public entity designed to work with local and provincial governments? What kind of entity will bridge the interests of donors with those of local authorities? Would donor organizations work directly with the proposed entity, reviewing accounts, evaluating performance, etc. or would an intermediary institution (e.g. an NGO) be necessary?

Which of the various alternatives are most likely to attract support from a consortium of bilateral and multilateral donors? Who are the potential participants in such a consortium? What are their requirements for participation?

\section{SECTION 3 -- Methodology}

Given the rather large range of issues the team must explore, and the complexity of a study that entails familiarity with agencies in Washington, the functioning of Agriculture and Rural Rehabilitation offices in Peshawar, Quetta, and in field offices in Afghanistan, and the need to explore alternative proposals with potential donors, the tasks will be divided into four discrete stages.

\section{Stage 1}

The first task will cover the period May 1 through July 30, 1991 and will be used by the Reconstruction Group as an information

$$
\begin{gathered}
N-3 / 9 \\
85
\end{gathered}
$$



gathering period. It is anticipated that during this time the MIT
team will:

- Familiarize itself with information available at A.I.D. and VITA on the existing ARR field operations. Information gathered will focus on but not be limited to:

* how projects are initiated, designed, reviewed, selected, implemented, monitored, and evaluated;

* the ARR's staffing pattern, recruitment scheme, compensation arrangements, training program, and level of managerial and technical skills;

* the relationship between ARRs and other programs and institutions, including other NGOs, international agencies and the local authorities with whom the ARRs interact at the local level. Prepare a briefing book with background materials on Afghan-
istan.

Meet with senior Afghan officials as well as representatives of USAID, State, World Bank and others who have first hand knowledge of Afghanistan or who can impart useful and relevant information for the purpose of this study. It is anticipated that these discussions will allow the team to understand how the VITA program fits into the overall AID program in Afghanistan; assess overall policy for the country in the relevant future; and gain some knowledge of historical and comparative examples of institutional arrangements for dealing with the reconstruction of rural infrastructures within the Afghani context.

\section{Stage 2}

The second stage will cover the period of July 31 to August 23 , 1991 and will comprise the team's first visit to Pakistan. During this time the MIT team will concentrate on the following:

a. Completion of its assessment of the existing ARR field operations, their organizational structure and manpower resources, and its relationship with local and provincial authorities. The task will concentrate on assessing the capacity of the existing staff and program to become an independent Afghan institution, and what is needed to create such an institution however its structure and mission are defined.

b. Meetings with officials of other NGOs in Peshawar with major responsibilities for rural reconstruction in

$$
86 \mathrm{~N}-4 / 9
$$


Afghanistan, both to hear their assessment of the ARR projects and to familiarize themselves with the scope of other rural reconstruction activities.

c. Meetings with local representatives of bilateral and multilateral donor organizations in Peshawar and Islamabad to review with them alternative proposals for converting the ARR organization into an independent Afghan-managed entity since their assessment and support is likely to be critical in whether or not their governments would take part in funding the proposed entity.

d. Preparation of a preliminary feasibility report so that feedback can be received from both VITA and USAID personnel. The report will describe the traditional, political, legal, and cultural constraints within which the proposed entity must function, report on the role played by other indigenous non-governmental entities in Afghanistan's development, suggest what missions might be performed by the proposed entity, indicate the overall structure of the proposed entity and how it might relate with local and regional authorities, with the government of Afghanistan, and with potential donors, indicate how the ARR could be converted into the proposed entity, and suggest how a consortium of donors might best be assembled. The report will also provide a preliminary plan for developing within the organization the managerial and technical skills necessary to plan and implement projects and to assure financial responsibility. While the team may recommend a single proposal, they have the option of presenting several alternative arrangements to VITA and USAID.

A tentative agenda for this trip is as follows:

July 31,1991

August 2, 1991

August $2 \& 3,1991$

August 3 afternoon

August $3-13,1991$

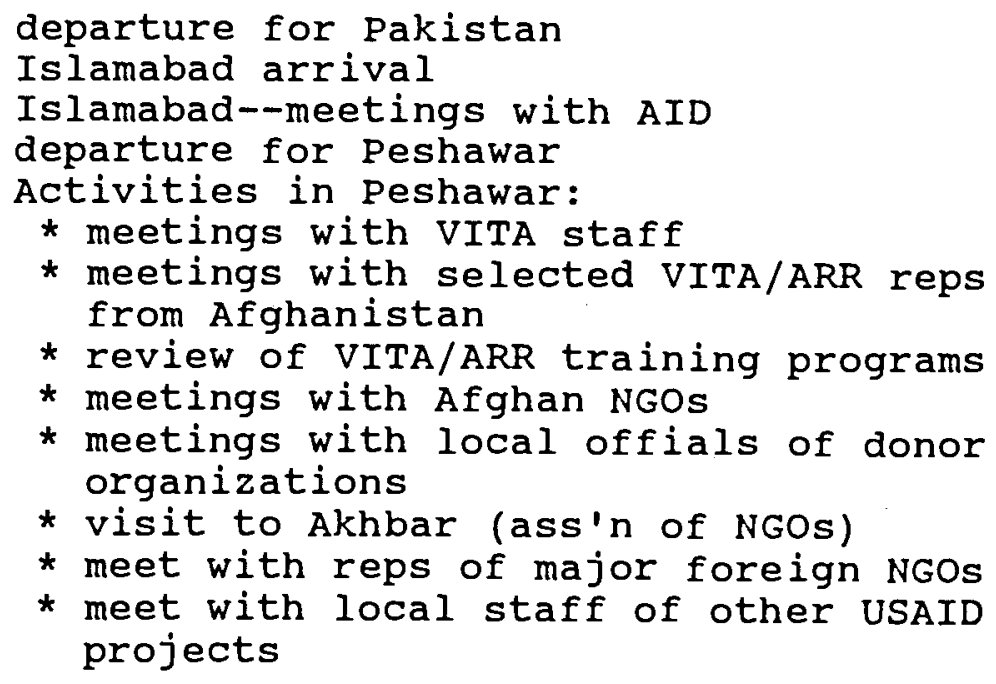
projects

$$
\text { N- } 5 / 9 \quad 87
$$


August 14, 1991

August $14 \& 15,1991$

August 16, 1991

August 17, 1991

August $18,19,20$

August 21, 1991

August 22, 1991

August 23, 1991
* meetings with appropriate Pakistani officials

Depart for Islamabad

Meetings with donors in Islamabad (UNDP UNOCA, CEDA, Swedish Committee, UNHCR, WFP, Japanese, German, etc.)

Afternoor departure for Peshawar

Meetings in Peshawar

Report preparation

Briefing/report presentation to VITA staff in Peshawar

Briefing/report presentation to USAID staff in Islamabad

Departure for the U.S.

\section{Stage 3}

Upon returning to the U.S. the MIT team will work to organize a resource conference in Washington DC, Boston or New York. The conference is to be held in the Fall of 1991 and serve as a forum for bringing together donor representatives, as well as senior Afghan officials for discussion of its recent findings in Pakistan and further analysis of funding potential.

Conference participants will include representatives from relevant United Nations Agencies, including the United Nations Development Fund (UNDP) the International Fund for Agricultural Development (IFAD), and UNOCA; bilateral donors, including representatives of the Official Development Assistance (ODA) program in Japan (e.g. representatives from JICA and OECF), the Canadian International Development Assistance program (CIDA), USAID, and various Arab funds for development; and the World Bank.key objective of the meeting will be to determine what the requirements are for the various donors, whether a consortium for creating an Afghan fund for rural reconstruction and development is feasible, and which of several institutional arrangements would be most attractive to donors.

Senior Afghan officials that will be invited will include but not be limited to the following: Mr. Abdul Tawab Assifi, former Minister of Mines and Industry; Mr. Ghulam Ali Ayeen, former Governor and Minister of Education; Mr. A. Siraj, former Minister of Health; Dr. Noor Ali, former Minister of Commerce and former President of Afghanistan's Industrial Bank; and Mr. Abdul Aziz Ferough, former Deputy Minister of Planning.

It should be noted that members of the Reconstruction Group and other faculty at MIT are reasonably knowledgeable as to what agencies and individuals might appropriately be invited, though the final list of invitees would be prepared in consultation with USAID and VITA.

$$
N-6 / 9 \quad 88
$$


MIT participants will include Professor Ralph Gakenheimer, Professor of Urban Planning and Civil Engineering; Professor Philip S. Khoury, Acting Dean, School of Humanities and Social Sciences; Professor Fred Moavenzadeh, Department of Civil Engineering and Director of the Center for Construction Research and Education; Professor Nazli Choucri, Department of Political Science and Associate Director, Technology and Development Program; and Professor Myron weiner, Department of Political Science and Director, Center for International studies. other Cambridge area academic participants will include Professor Ali Banuazizi, Department of Psychology, Boston College and Research Fellow, Center for International studies; and Professor Tom Barfield, Chair, Department of Anthropology, Boston University.

Background materials on the existing VITA/USAID reconstruction program in Afghanistan will be distributed to the conference participants, along with a background paper describing the issues which must be addressed in creating a consortium of donors. Upon completion of the conference, a report will be submitted to VITA and USAID which will specify the requirements, priorities and preferences of various donoros as well as the institutional arrangements they would consider most attractive. The report will provide an essential input to the team's efforts to find a balance among donor preferences, Afghanistan realities and feasible institutional options.

\section{Stage 4}

The fourth stage will mainly require a second visit to Islamabad/Peshawar. The primary purpose of this second visit will be to meet with local representatives of donor institutions and with VITA and USAID and review the final findings of the Reconstruction Group. This brief trip will be a brief trip and it will take place no later than January, 1992 .

The final report that will be prepared by MIT will include a description of the traditional, political, legal, and cultural constraints within which the proposed entity must function, report on the role played by other indigeneous non-governmental entities in Afghanistan's development, suggest what missions might be performed by the proposed entity, indicate the overall structure of the proposed entity and how it might relate with local and regional authorities, with the government of Afghanistan, and with potential donors, indicate how the ARR could be converted into the proposed entity, and suggest how a consortium of donors might best be assembled. The report will also provide a preliminary plan for developing within the organization the managerial and technical skills necessary to plan and implement projects and to assure financial responsibility. While the team may recommend a single proposal, they leave open the possibility that several alternative arrangements might be suggested to VITA and to USAID.

$$
N-7 / 9 \quad 89
$$


Proposed work on the feasibility study will commence on May 1 , 1991 and be completed by March 31, 1992.

A part-time staff coordinator will be appointed to organize the conference of potential donors, provide overall administrative support and be the administrative contact point for VITA. A graduate student will be appointed to assist in the preparation of background materials. The coordinator, with part-time secretarial and graduate student assistance, will take responsibility for the logistics involved in arranging briefings in washington, distributing materials to the team, preparing for the meeting with potential donors, getting the team to pakistan, and assisting in report preparation. The subcontract budget provides for all the costs associated with the conference and for the relevant administrative costs and operating expenses other than travel by team members to Washington and to Pakistan.

\section{BECTION 5 -- List of Proposed consultants}

Listed below are the proposed consultants for participation in the feasibility study. It is our understanding that VITA and AID will make the final selection, and may request the names of additional or alternative consultants. VITA and AID will negotiate appropriate compensation for each of the consultants, as well as provide for per diems and travel expenses. A budget is submitted indicating the number of days and compensation proposed for each of the consultants:

Ali Banuazizi, Research Affiliate, Center for International Studies and Professor, Department of Psychology, Boston College and co-director of the MIT project on the state and restructuring of society in Afghanistan, Pakistan and Iran, with UN consulting experience on developing social indicators for evaluating programs and policies.

Professor Thomas Barfield, Chair, Department of Anthropology, Boston University, a rural anthropologist with expertise on Afghan social organization, who has done considerable consulting work relating to Afghanistan.

Nazli Choucri, Professor of Political Science and Associate Director of the MIT Technology and Development Program, with expertise on international organizations and development.

Ralph Gakenheimer, Professor of Urban Planning and civil Engineering, MIT, a specialist on infrastructures in developing countries, with considerable experience in Arab countries and in Latin America.

$$
\mathrm{N}-8 / 990
$$


Philip s. Khoury, Acting Dean, School of Humanities and Social science, Professor of History with knowledge of the comparative history of post-war reconstructions, and a specialist on the role of tribes in Midale East state formation.

Fred Moavenzadeh, Director, Center for Construction Research and Education, Department of Civil Engineering, experienced in the design of engineering organizations in developing countries.

Jerome Rothenberg, Professor of Economics, MIT, a specialist in urban economics with expertise in the analysis of structural change and the financing of infrastructure services.

Myron weiner, Director of the Center for International studies and Ford International Professor of Political Science, co-director of the Ford Foundation-funded project at MIT on the state and restructuring of society in Afghanistan, Pakistan and Iran, with previous experience consulting on institutional development programs, policies, and evaluations in South and Southeast Asia.

While all members of the team will contribute to all aspects of the study, individual members will take initial responsibility for preparing specific sections of the feasibility report. Professors Barfield and Banuazizi will take responsibility for describing the traditional political, legal and cultural constraints within which the proposed entity must function. Professor Khoury will indicate how the proposed entity might relate to local and regional authorities, including an exploration of the relevant legal issues. Professors Gakenheimer and Moavenzadeh will provide a preliminary plan for developing within the organization the managerial and technical skills necessary to plan and implement projects. Professors Choucri and Weiner will focus on the relationship between the proposed entity and potential donors, their requirements, priorities and preferred institutional arrangements. Professor Weiner, as director of the team, will take responsibility for final report preparation, with support from the team's administrative coordinator. Because of the individual areas of expertise and the division of labor anticipated in the preparation of the final report, it is not anticipated that consultants will be participating in both trips. 


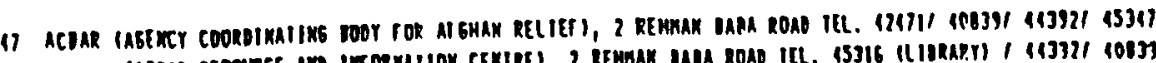

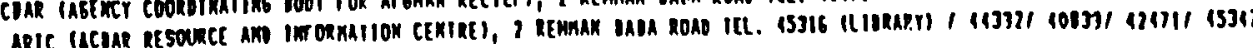

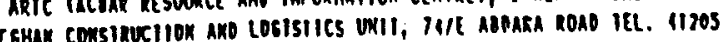

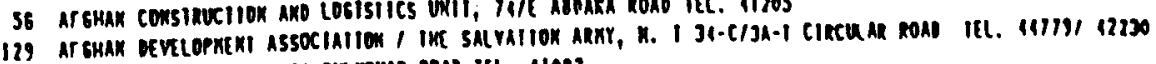

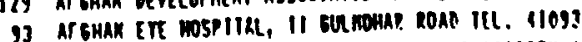

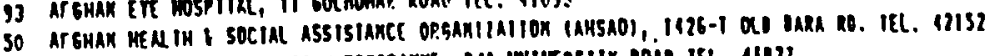

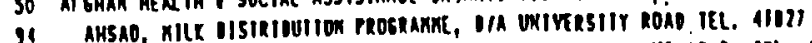

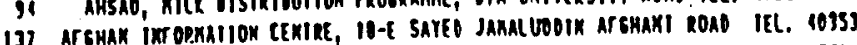

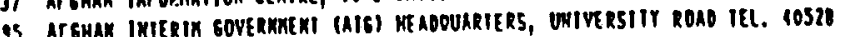

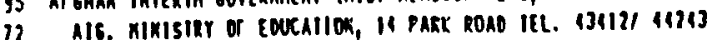

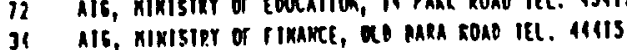

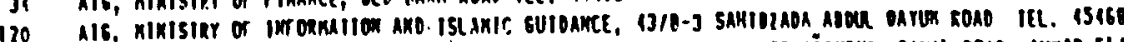

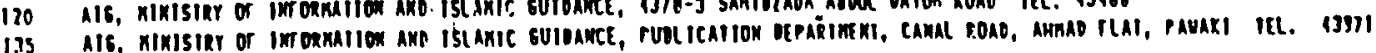

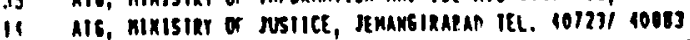

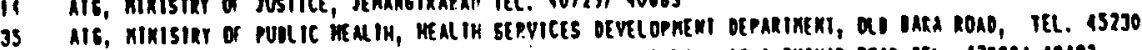

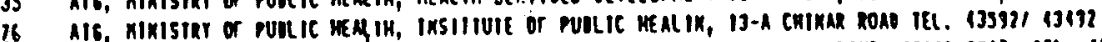

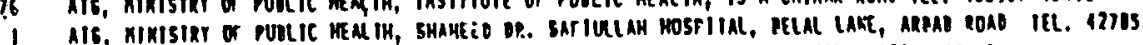

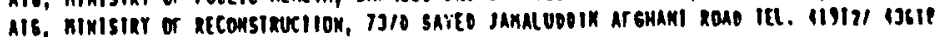

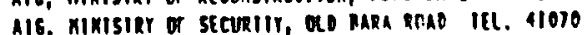

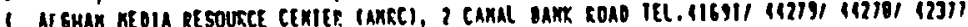

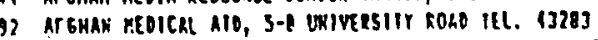

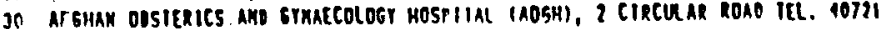

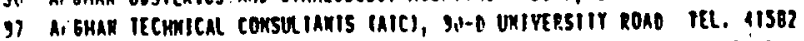

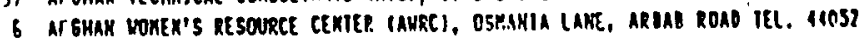

115 AT GHAMAID, 3-1 SUR KOHAR ROAO IEL. 12030; $13731 / 12322$

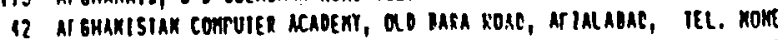

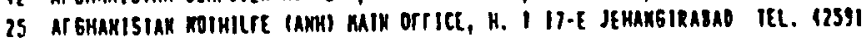

13 AFRAKE, WHIE ROUSt, I PARK LANE IEL. 12320

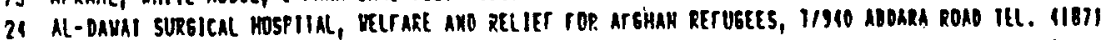

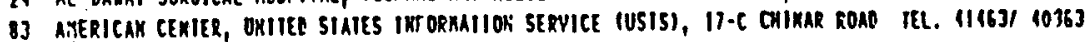

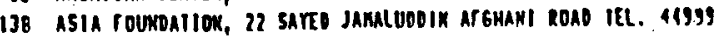

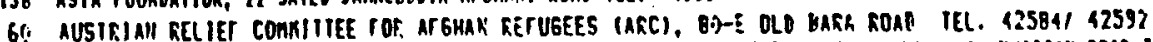

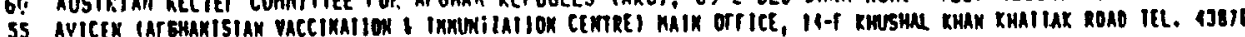

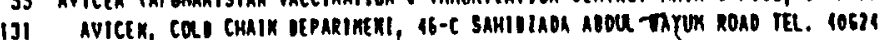

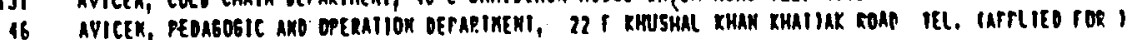

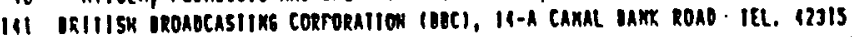

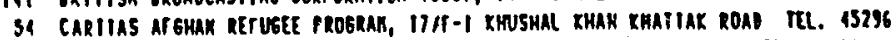

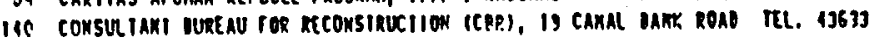

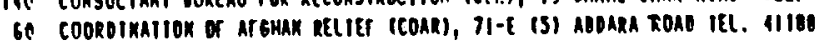

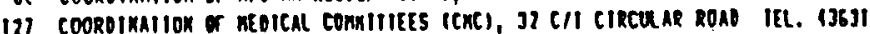

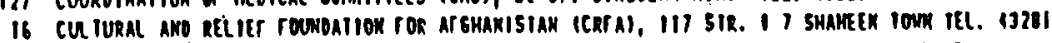

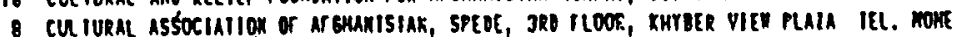

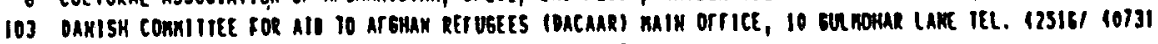

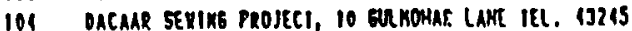

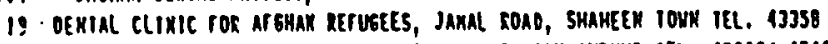

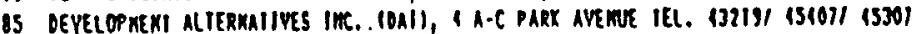

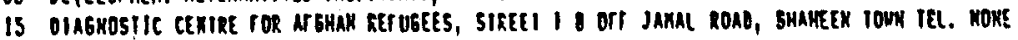

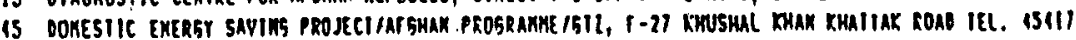

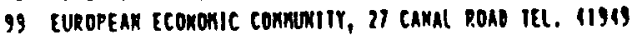

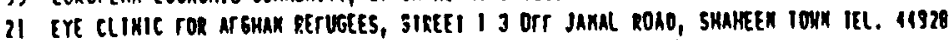

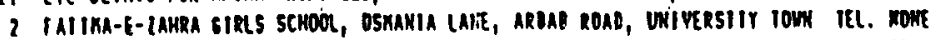

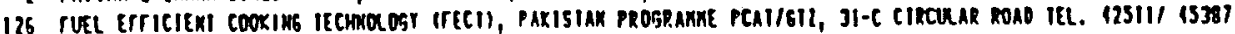

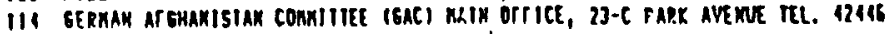

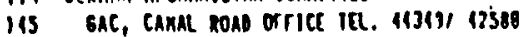

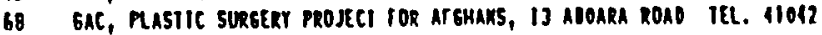

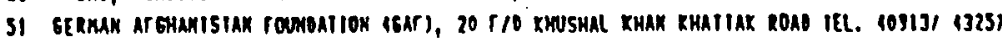

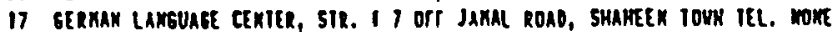

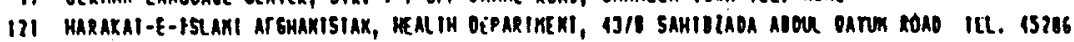

113 HELP IGERAXYI, J1-E SATEI JAMALUBOIM ATGHAXI ROAB IEL. 11530

65 HELF THE Ar GHAKS FOUROAIOX, S-C/2 ABOAKA ROAO IEL. (10OS3

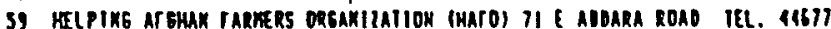

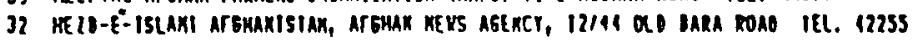

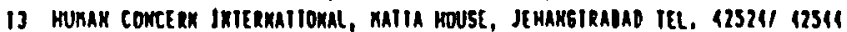

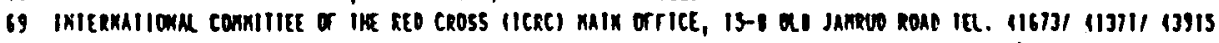

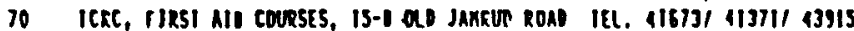

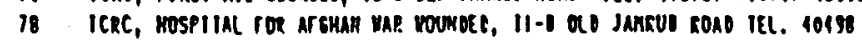

13: ICRC, OKIHOPAEQHC CENIRE IOC AF SHANS, 62-E CAKAL ROAO IEL. (I)

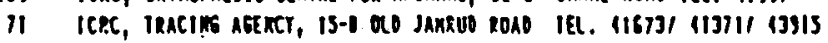

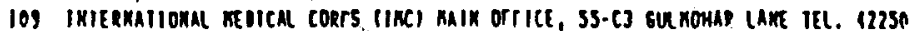

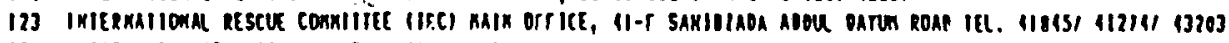

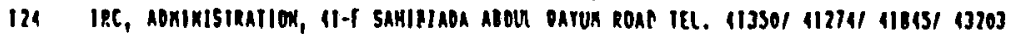

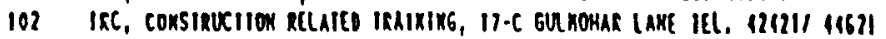

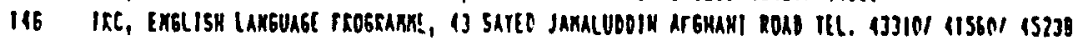

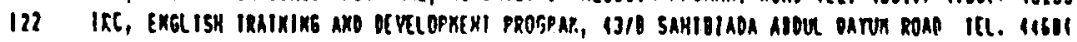

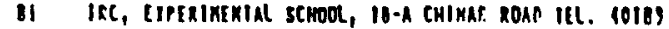

33 IRC, HAMOICRATIS - SELF RELIAKCE FrOJCCI, OCO DAKA RORO TEL. 12565

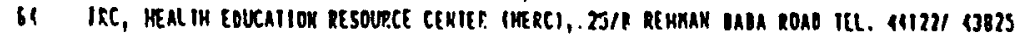




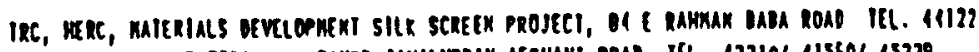

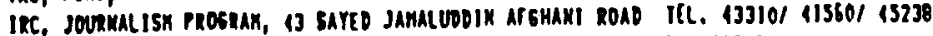

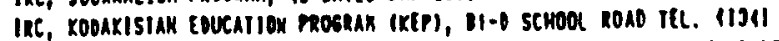

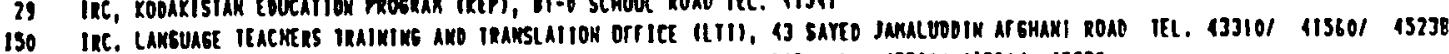

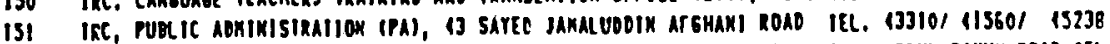

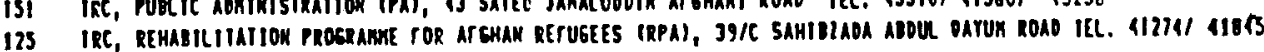

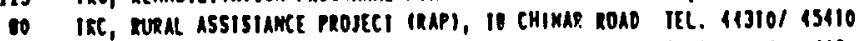

17 IRC, SCIEKCE ANO IECHNOLOSY TRATMING ISTII, 37-1 CHIMAR ROAO IEL. 11314

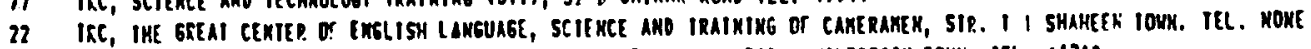

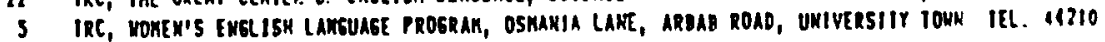

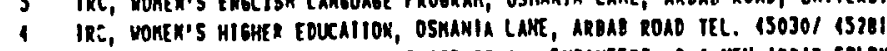

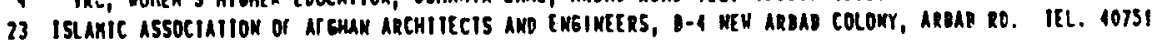

II6 ISLAFIC COOROINAIIOA COUACIL, J GLKOHAR rOAO IfL. 12241

62 ISLAKIC IKIERNAITONAL RELIFP ORGAMIIATION IIIRO), 68/E2 AOBARA ROAO TEL. 11322

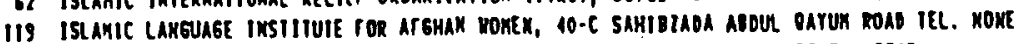

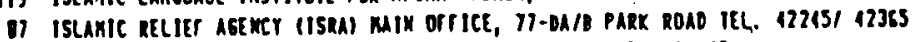

is ISRA, ORPHANS WeC

7) ISkA, PSTChIATRIC CIINIC, SA/I PARK ROAO, TEL. NOME

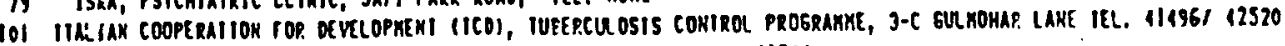

128 JAPAK-AT GHAK GEDICAL SERVICES, J-C/2 CIRCULAR ROAD TEL. $11350 / 41341$

9 JEHAO UNIVERSIIY FACULIY Of EOUCAIIOX, JS PARA ROAO IEL. ROHE

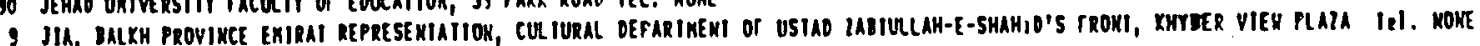

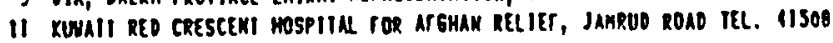

108 naDERa, 33-C II GULnOHAR LAME TEL. 42234

39 MANAEKEXI SCIERCES TOR MELIH (HSH), OLO BARA ROAO TEL. 10792/ $12867 /$ 11564

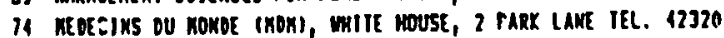

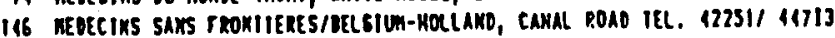

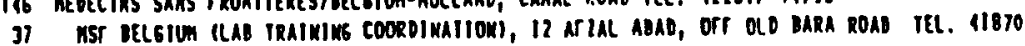

36 nerer rUNB, kHATtaK hOUSE, OC BARA nOAO IEL. 12105

56 nUSLI AID (UK) CEMTRAL REICAL LAB, 12 f/A XHUSHAL KHAK KHATIAK ROAD IEL. 13326

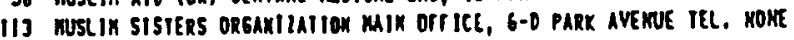

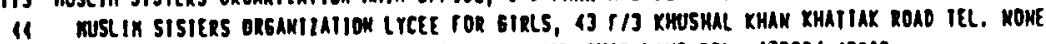

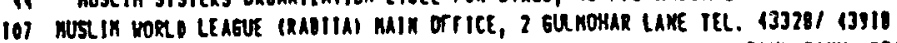

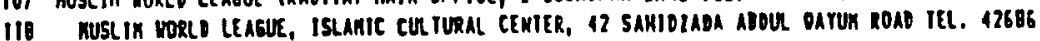

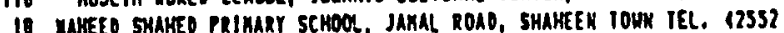

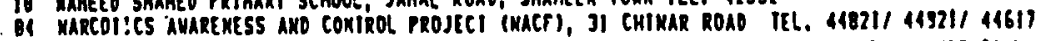

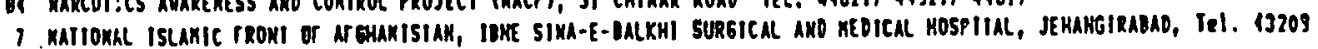

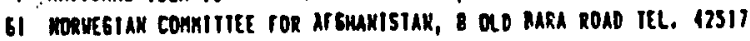

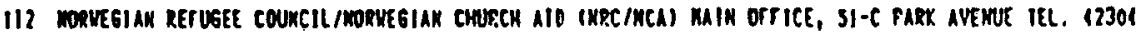

OB MRCIUCA, PROJECI OrTICE, 3-1 JAKRUD ROAO IEL. 11129/ 15210

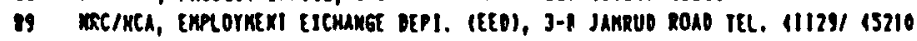

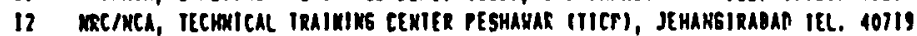

JI OCKENOEK VEXIURE, 1210-I OLO JAKRUO ROAD TEL. 10410

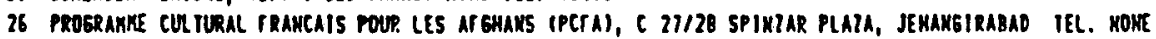

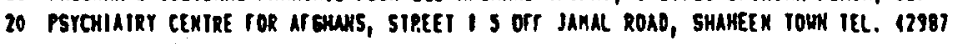

147 RADOA DARAEK (SUEDISH SAVE the CRILOREN), J CAMAL ROAO IEL. 43905

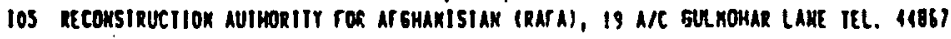

J REMAIIIIIION CERTRE FOR IISALLEO AF GHAK RETUGEES, I/87S AREAB COLONY, ARBAP ROAO IEL. 41032 SALVAIION ARKT (SEE AF GHAN, OEYELOPNEXI ASSOCIATION, I 129)

IIT SAUOI REO CRESCEMT SOCIETY, 2 GUROHAR ROAO IEL. 12207

I12 SAVE IKE CHILDEE (UK), 39-1 SAYEO JAKAL UDDIX AFôHAHI R.OAO IEL. (11918/ 11170

136 SAVE THE CHILBEN (US), 16-E AIIATURK (AKE IEL. 13219/ 12462

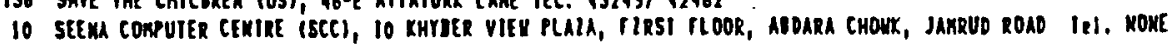

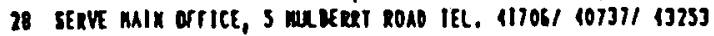

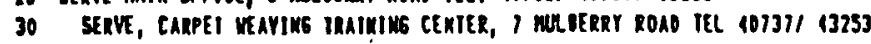

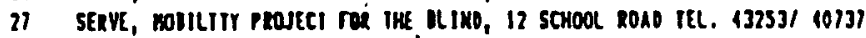

66 soc indeite arshaxistak, it II/6 piak rodo. 41121

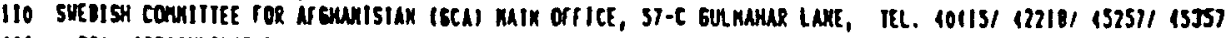

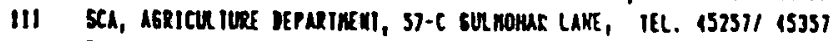

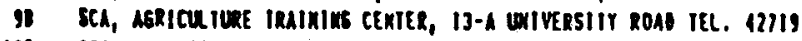

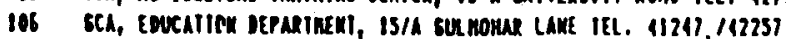

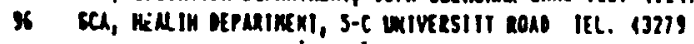

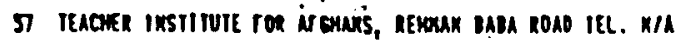

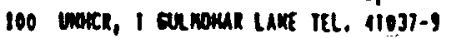

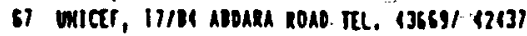

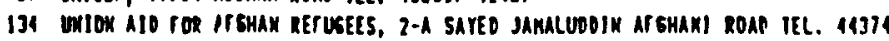

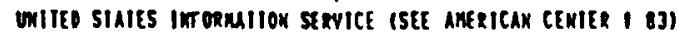

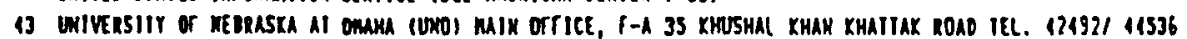

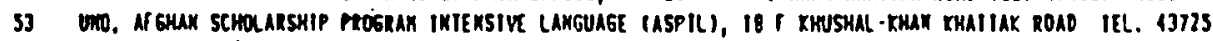

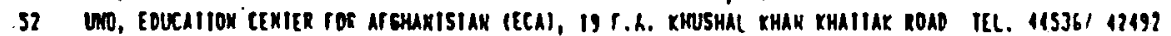

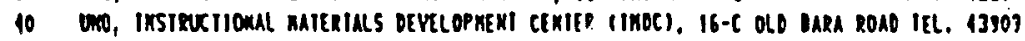

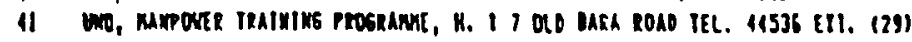

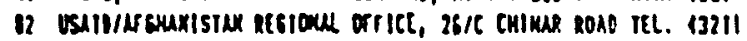

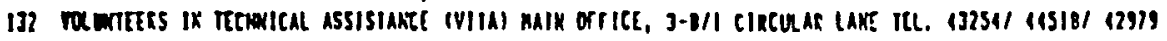

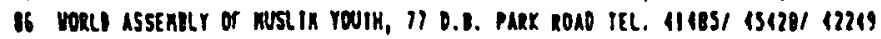

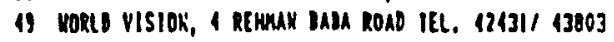




\section{SCOPE OF WORR}

Consultant: Mr. Aldelmo Ruiz

As a consultant to the VITA/Peshawar Office, Mr. Ruiz will be responsible for the following:

1. Examining roject records and interviewing field personnel to gain an understandirig of profect hlstory and developmer.t. Reviewing production records with reference to the first year Consolidated Annual Implementation Plan to understand which areas need revision.

2. Working with the fleld staff and headquarters staff in preparing the Implementation Plan for the second year. The writing of the plan will be done by the consultant with input from VITA staff and with review and approal by the Chief of Party.

3. Other such appropriate assignments as might be given by the Chief of Party.

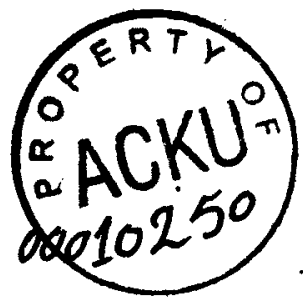

$$
94
$$

